\title{
ASYMPTOTIC POINTWISE BEHAVIOR FOR SYSTEMS OF SEMILINEAR WAVE EQUATIONS IN THREE SPACE DIMENSIONS
}

\author{
SOICHIRO KATAYAMA
}

\begin{abstract}
In connection with the weak null condition, Alinhac introduced a sufficient condition for global existence of small amplitude solutions to systems of semilinear wave equations in three space dimensions. We introduce a slightly weaker sufficient condition for the small data global existence, and we investigate the asymptotic pointwise behavior of global solutions for systems satisfying this condition. As an application, the asymptotic behavior of global solutions under the Alinhac condition is also derived.
\end{abstract}

\section{INTRODUCTION}

This paper is devoted to the study of the Cauchy problem for systems of semilinear wave equations in three space dimensions. Throughout this paper, for the variables $t \in \mathbb{R}$ and $x=\left(x_{1}, x_{2}, x_{3}\right) \in \mathbb{R}^{3}$, we use the notation

$$
\partial_{0}=\partial_{t}=\frac{\partial}{\partial t}, \quad \partial_{k}=\frac{\partial}{\partial x_{k}}, k=1,2,3 .
$$

$\Delta_{x}$ and $\square$ denote the Laplacian and the d'Alembertian, respectively; namely we define $\Delta_{x}=\sum_{k=1}^{3} \partial_{k}^{2}$ and $\square=\partial_{t}^{2}-\Delta_{x}$. For a matrix (or vector) $\mathbf{B}$, its transpose is written as $\mathbf{B}^{\mathrm{T}}$.

We consider the Cauchy problem for systems of semilinear wave equations of the type

$$
\square u_{j}=F_{j}(u, \partial u) \quad \text { in }(0, \infty) \times \mathbb{R}^{3}, \quad j=1,2, \ldots, N
$$

with initial data

$$
u(0, x)=\varepsilon f(x),\left(\partial_{t} u\right)(0, x)=\varepsilon g(x) \text { for } x \in \mathbb{R}^{3},
$$

where $u=\left(u_{1}, \ldots, u_{N}\right)^{\mathrm{T}}$ and $\partial u=\left(\partial_{0} u, \partial_{1} u, \partial_{2} u, \partial_{3} u\right)$. Here each $u_{j}$ is supposed to be a real-valued function of $(t, x) \in[0, \infty) \times \mathbb{R}^{3}$. We assume that $f=\left(f_{j}\right)_{1 \leq j \leq N}^{\mathrm{T}}$, $g=\left(g_{j}\right)_{1 \leq j \leq N}^{\mathrm{T}} \in C_{0}^{\infty}\left(\mathbb{R}^{3} ; \mathbb{R}^{N}\right)$ in (1.2), and that $\varepsilon$ is a small and positive parameter. We refer to $(f, g)$ as the initial profile in what follows. Throughout this paper, we always assume for simplicity that each $F_{j}$ with $1 \leq j \leq N$ is a homogeneous polynomial of degree 2 in its arguments $(u, \partial u)$.

We say that the small data global existence (or SDGE in short) holds for (1.1) if for any $f, g \in C_{0}^{\infty}\left(\mathbb{R}^{3} ; \mathbb{R}^{N}\right)$, there exists a positive constant $\varepsilon_{0}$ such that for any $\varepsilon \in\left(0, \varepsilon_{0}\right.$ ] the Cauchy problem (1.1)-(1.2) possesses a global classical solution $u \in$ $C^{\infty}\left([0, \infty) \times \mathbb{R}^{3} ; \mathbb{R}^{N}\right)$. It is known that SDGE does not hold for general quadratic 
nonlinearity (see John [8] and [9]). Klainerman [16] introduced the null condition, and proved SDGE for (1.1) under the null condition (see also Christodoulou [5]). To state the null condition, we introduce the reduced nonlinearity

$$
F_{j}^{\mathrm{red}}(\omega, X, Y):=F_{j}\left(X,\left(-Y, \omega_{1} Y, \omega_{2} Y, \omega_{3} Y\right)\right), \quad 1 \leq j \leq N
$$

for $\omega=\left(\omega_{1}, \omega_{2}, \omega_{3}\right) \in S^{2}, X=\left(X_{1}, \ldots, X_{N}\right)^{\mathrm{T}} \in \mathbb{R}^{N}$, and $Y=\left(Y_{1}, \ldots, Y_{N}\right)^{\mathrm{T}} \in \mathbb{R}^{N}$, where $S^{2}$ denotes the unit sphere in $\mathbb{R}^{3}$. In other words, $F_{j}^{\text {red }}$ is obtained by substituting $X_{k}$ and $\omega_{a} Y_{k}$ (with $1 \leq k \leq N$ and $0 \leq a \leq 3$ ) for $u_{k}$ and $\partial_{a} u_{k}$ in $F_{j}(u, \partial u)$, respectively, where $\omega_{0}=-1$. We say that $F=\left(F_{j}\right)_{1 \leq j \leq N}^{\mathrm{T}}$ satisfies the null condition if

$$
F_{j}^{\mathrm{red}}(\omega, X, Y)=0, \quad 1 \leq j \leq N, X, Y \in \mathbb{R}^{N}, \omega \in S^{2} .
$$

We introduce the null forms

$$
\begin{aligned}
Q_{0}(\varphi, \psi) & :=\left(\partial_{t} \varphi\right)\left(\partial_{t} \psi\right)-\sum_{k=1}^{3}\left(\partial_{k} \varphi\right)\left(\partial_{k} \psi\right), \\
Q_{a b}(\varphi, \psi) & :=\left(\partial_{a} \varphi\right)\left(\partial_{b} \psi\right)-\left(\partial_{b} \varphi\right)\left(\partial_{a} \psi\right), \quad 0 \leq a<b \leq 3 .
\end{aligned}
$$

When each $F_{j}$ is a homogeneous polynomial of degree 2 in $(u, \partial u)$, as is assumed, we can show that $F=\left(F_{j}\right)_{1 \leq j \leq N}^{\mathrm{T}}$ satisfies the null condition if and only if $F$ can be written as

$$
F_{j}(u, \partial u)=\sum_{k, l=1}^{N}\left(r_{j}^{0, k l} Q_{0}\left(u_{k}, u_{l}\right)+\sum_{a, b=0}^{3} r_{j}^{a b, k l} Q_{a b}\left(u_{k}, u_{l}\right)\right), \quad 1 \leq j \leq N
$$

with some constants $r_{j}^{0, k l}$ and $r_{j}^{a b, k l}$ (see [16] for instance). We can also show that if the null condition is satisfied, then the global solution $u$ to (1.1)-(1.2) for small $\varepsilon$ is asymptotically free in the energy sense, that is to say, there exists a solution $\widetilde{u}$ to the free wave equation $\square \widetilde{u}=0$ with some data in $\dot{H}^{1}\left(\mathbb{R}^{3}\right) \times L^{2}\left(\mathbb{R}^{3}\right)$ such that

$$
\lim _{t \rightarrow \infty}\|(u-\widetilde{u})(t, \cdot)\|_{E}=0
$$

where $\dot{H}^{1}\left(\mathbb{R}^{3}\right)$ is the homogeneous Sobolev space, and the energy norm $\|\cdot\|_{E}$ is given by

$$
\|\varphi(t, \cdot)\|_{E}:=\left(\frac{1}{2} \int_{\mathbb{R}^{3}}\left(\left|\partial_{t} \varphi(t, x)\right|^{2}+\left|\nabla_{x} \varphi(t, x)\right|^{2}\right) d x\right)^{1 / 2} .
$$

Since we have the conservation of the energy $\|\widetilde{u}(t, \cdot)\|_{E}=\|\widetilde{u}(0, \cdot)\|_{E}$ for the free solution $\widetilde{u}$, (1.7) implies

$$
\lim _{t \rightarrow \infty}\|u(t, \cdot)\|_{E}=\|\widetilde{u}(0, \cdot)\|_{E} \cdot
$$

In order to understand the null condition, we introduce the reduced system. For this purpose, first we recall the asymptotic behavior of the free solution: Let 
$\varphi, \psi \in C_{0}^{\infty}\left(\mathbb{R}^{3} ; \mathbb{R}\right)$, and consider the Cauchy problem for the single wave equation

$$
\begin{array}{ll}
\square u_{0}(t, x)=0 & \text { for }(t, x) \in(0, \infty) \times \mathbb{R}^{3}, \\
u_{0}(0, x)=\varphi(x), \quad\left(\partial_{t} u_{0}\right)(0, x)=\psi(x) & \text { for } x \in \mathbb{R}^{3} .
\end{array}
$$

It is well known that the solution $u_{0}$ to (1.10)-(1.11) can be written as

$$
u_{0}(t, x)=\frac{1}{4 \pi t} \int_{|y-x|=t} \psi(y) d S_{y}+\partial_{t}\left(\frac{1}{4 \pi t} \int_{|y-x|=t} \varphi(y) d S_{y}\right),
$$

where $d S_{y}$ is the area element of the sphere with radius $t$ centered at $x$. Suppose that

$$
\operatorname{supp} \varphi \cup \operatorname{supp} \psi \subset B_{R}:=\left\{x \in \mathbb{R}^{3} ;|x| \leq R\right\}
$$

with a positive constant $R$. Then (1.12) implies that $u_{0}(t, x)=0$ for any $(t, x) \in$ $[0, \infty) \times \mathbb{R}^{3}$ satisfying $|r-t| \geq R$ with $r=|x|$ (this property is called the (strong) Huygens principle). Hence it is reasonable to consider the asymptotic behavior of $u_{0}$ for large $t$ (or equivalently large $r$ ) with $r-t$ being fixed; then we can expect that the integrals over the sphere of radius $t$ centered at $x=r \omega$ (with $\omega=|x|^{-1} x$ ) in (1.12) tend to those over the tangential plane of the sphere at the point $(r-t) \omega$. This is the motivation to introduce the Friedlander radiation field

$$
\mathcal{F}_{0}[\varphi, \psi](\sigma, \omega)=\frac{1}{4 \pi}\left(\mathcal{R}[\psi](\sigma, \omega)-\left(\partial_{\sigma} \mathcal{R}[\varphi]\right)(\sigma, \omega)\right)
$$

for $(\sigma, \omega) \in \mathbb{R} \times S^{2}$, where $\mathcal{R}[h]$ is the Radon transform of a function $h$ given by

$$
\mathcal{R}[h](\sigma, \omega)=\int_{y \cdot \omega=\sigma} h(y) d S_{y}^{\prime} .
$$

Here $d S_{y}^{\prime}$ is the area element of the plane $\left\{y \in \mathbb{R}^{3} ; y \cdot \omega=\sigma\right\}$. It is known that for the solution $u_{0}$ to (1.10)-(1.11) we have

$$
\begin{aligned}
& \left|r u_{0}(t, r \omega)-\mathcal{F}_{0}[\varphi, \psi](r-t, \omega)\right| \\
& \quad+\sum_{a=0}^{3}\left|r\left(\partial_{a} u_{0}\right)(t, r \omega)-\omega_{a}\left(\partial_{\sigma} \mathcal{F}_{0}[\varphi, \psi]\right)(r-t, \omega)\right| \leq C(1+t+r)^{-1}
\end{aligned}
$$

for any $\omega=\left(\omega_{1}, \omega_{2}, \omega_{3}\right) \in S^{2}$ and any $(t, r)$ satisfying $r \geq t / 2 \geq 1$, where we have set $\omega_{0}=-1$ as before, and $C$ is a positive constant determined by $\varphi$ and $\psi$ (see Hörmander [7, Theorem 6.2.1] for instance; see also Katayama-Kubo [13]).

Let $u$ be the solution to (1.1)-(1.2). Motivated by the Friedlander radiation field, and taking account of the lifespan of the solution for the case of general quadratic nonlinearity, we seek an approximation of the solution $u$ of the form

$$
\varepsilon r^{-1} U(\varepsilon \log t, r-t, \omega),
$$

which approximates $u(t, r \omega)$ as $\varepsilon$ tends to 0 , with $\tau=\varepsilon \log t, \sigma=r-t$, and $\omega\left(\in S^{2}\right)$ being fixed. Formal calculations show that $U=U(\tau, \sigma, \omega)=\left(U_{j}(\tau, \sigma, \omega)\right)_{1 \leq j \leq N}^{\mathrm{T}}$ 
should be determined by

$$
-2 \partial_{\tau} \partial_{\sigma} U_{j}(\tau, \sigma, \omega)=F_{j}^{\mathrm{red}}\left(\omega, U(\tau, \sigma, \omega), \partial_{\sigma} U(\tau, \sigma, \omega)\right), \quad 1 \leq j \leq N
$$

which is called the reduced system (the quasi-linear version of this system was successfully used in the detailed study of the lifespan of the solution; see John [11] and Hörmander [6]). Now we understand that the null condition (1.3) says that the right-hand side of (1.16) vanishes identically, and we can solve (1.16) globally. From this observation, Lindblad-Rodnianski [21] introduced the notion of the weak null condition, which means that the reduced system always has global solutions with at most exponential growth in $\tau$ (see also [22]); it is conjectured that the weak null condition implies SDGE, but this conjecture is still open.

In connection with the weak null condition, Alinhac [3] considered the case of $F=F(\partial u)$, and introduced a sufficient condition for SDGE, which is stronger than the weak null condition, but still weaker than the null condition. For simplicity of exposition, when $F_{j}=F_{j}(\partial u)$, we omit $X$ in $F_{j}^{\text {red }}(\omega, X, Y)$ and write $F_{j}^{\text {red }}=$ $F_{j}^{\text {red }}(\omega, Y)$ for $1 \leq j \leq N$ in what follows. If we write $\omega_{0}=-1, \omega=\left(\omega_{1}, \omega_{2}, \omega_{3}\right) \in$ $S^{2}$, and $Y=\left(Y_{j}\right)_{1 \leq j \leq N}^{\mathrm{T}} \in \mathbb{R}^{N}$, then his condition can be read as follows:

Condition 1.1 (Alinhac [3]). There exist a real valued function $M=M(\omega, Y)$, an $\mathbb{R}^{N}$-valued function $\beta=\beta(\omega)=\left(\beta_{j}(\omega)\right)_{1 \leq j \leq N}^{\mathrm{T}}$, a positive integer $N_{0}$, and linear forms $g_{j l}=g_{j l}(\omega, Y)$ and $h_{l}=h_{l}(\omega, Y)$ in $Y$, which can be written as

$$
\begin{aligned}
g_{j l}(\omega, Y)=\sum_{k=1}^{N} g_{j l, k}(\omega) Y_{k}, & 1 \leq j \leq N, 1 \leq l \leq N_{0}, \\
h_{l}(\omega, Y)=\sum_{a=0}^{3} \sum_{k=1}^{N} h_{l, k a} \omega_{a} Y_{k}, & 1 \leq l \leq N_{0}
\end{aligned}
$$

with smooth coefficients $g_{j l, k}=g_{j l, k}(\omega)$ and real constants $h_{l, k a}$, such that

$$
\begin{array}{ll}
F_{j}^{\mathrm{red}}(\omega, Y)=M(\omega, Y) \beta_{j}(\omega), & 1 \leq j \leq N,(\omega, Y) \in S^{2} \times \mathbb{R}^{N}, \\
F_{j}^{\mathrm{red}}(\omega, Y)=\sum_{l=1}^{N_{0}} g_{j l}(\omega, Y) h_{l}(\omega, Y), & 1 \leq j \leq N,(\omega, Y) \in S^{2} \times \mathbb{R}^{N}, \\
h_{l}(\omega, \beta(\omega))=0, & 1 \leq l \leq N_{0}, \omega \in S^{2} .
\end{array}
$$

Remark 1.2. (1.19), (1.20), and (1.21) yield

$$
M(\omega, \beta(\omega))=0, \quad \omega \in S^{2} .
$$

The weak null condition follows from (1.19) and (1.22), but it is not known if these two conditions (1.19) and (1.22) are sufficient for SDGE (see Alinhac [3]). 
It is easy to see that the null condition implies Condition 1.1. A simple example satisfying Condition 1.1 but not the null condition is

$$
\left\{\begin{array}{l}
\square u_{1}=\left(\partial_{1} u_{1}\right)\left(\partial_{1} u_{2}-\partial_{2} u_{1}\right), \\
\square u_{2}=\left(\partial_{2} u_{1}\right)\left(\partial_{1} u_{2}-\partial_{2} u_{1}\right)
\end{array}\right.
$$

in $(0, \infty) \times \mathbb{R}^{3}$. We have $F_{1}^{\mathrm{red}}=\omega_{1} Y_{1}\left(\omega_{1} Y_{2}-\omega_{2} Y_{1}\right)$ and $F_{2}^{\mathrm{red}}=\omega_{2} Y_{1}\left(\omega_{1} Y_{2}-\omega_{2} Y_{1}\right)$, and we find that Condition 1.1 is satisfied with

$$
\begin{aligned}
& M(\omega, Y)=Y_{1}\left(\omega_{1} Y_{2}-\omega_{2} Y_{1}\right), \beta(\omega)=\left(\omega_{1}, \omega_{2}\right)^{\mathrm{T}}, \\
& N_{0}=1, g_{11}(\omega, Y)=\omega_{1} Y_{1}, g_{21}(\omega, Y)=\omega_{2} Y_{1}, h_{1}(\omega, Y)=\omega_{1} Y_{2}-\omega_{2} Y_{1} .
\end{aligned}
$$

If we put $w=\partial_{1} u_{2}-\partial_{2} u_{1}$, which corresponds to $h_{1}$ above, then we obtain

$$
\left\{\begin{array}{l}
\square u_{1}=w\left(\partial_{1} u_{1}\right), \\
\square u_{2}=w\left(\partial_{2} u_{1}\right), \\
\square w=\partial_{1} \square u_{2}-\partial_{2} \square u_{1}=Q_{12}\left(w, u_{1}\right) .
\end{array}\right.
$$

As we will see later, this hidden structure plays an important role in deriving global solutions. Concerning the asymptotic behavior, Katayama-Kubo [12] showed that global solutions under Condition 1.1 may not be asymptotically free in the energy sense: For example, it is shown that for some initial profile, there exists a positive constant $C$ such that

$$
\|u(t)\|_{E} \geq C \varepsilon(1+t)^{C \varepsilon} \quad \text { for small } \varepsilon,
$$

where $u=\left(u_{1}, u_{2}\right)^{\mathrm{T}}$ is the global solution to (1.23) (or equivalently $\left(u_{1}, u_{2}, w\right)^{\mathrm{T}}$ is the global solution to (1.24) ). The estimate (1.25) makes a sharp contrast to (1.9), and the solution $u$ cannot be asymptotically free in the energy sense for such data.

Now the following questions arise:

Question 1.3. We know that sometimes the global solution to (1.1) is asymptotically free, and sometimes not so with increasing energy. Do we have other kind of the asymptotic behavior? Especially, is there some nonlinearity $F$ such that the global solution to (1.1) for some small data behaves differently from free solutions, although its energy stays bounded from above and below by positive constants?

Question 1.4. In addition to Condition 1.1 from Alinhac [3], what condition do we need in order to ensure that the global solutions with small data behave like free solutions?

In this paper, motivated by the Friedlander radiation field, we will investigate the asymptotic pointwise behavior of global solutions for large $t$ with $r-t$ and $\omega$ being fixed, under a certain condition which is related to Condition 1.1, and we will answer the two questions above from this point of view.

Throughout this paper, as usual, various positive constants will be indicated just by the same letter $C$, and its actual value may change line by line. 


\section{The Main Results}

2.1. Notation. First we introduce some notation. We define the vector fields

$$
\begin{aligned}
S & :=t \partial_{t}+x \cdot \nabla_{x}, \\
L & =\left(L_{1}, L_{2}, L_{3}\right):=t \nabla_{x}+x \partial_{t}=\left(t \partial_{j}+x_{j} \partial_{t}\right)_{1 \leq j \leq 3}, \\
\Omega & =\left(\Omega_{1}, \Omega_{2}, \Omega_{3}\right):=x \times \nabla_{x}=\left(x_{2} \partial_{3}-x_{3} \partial_{2}, x_{3} \partial_{1}-x_{1} \partial_{3}, x_{1} \partial_{2}-x_{2} \partial_{1}\right), \\
\partial & :=\left(\partial_{0}, \partial_{1}, \partial_{2}, \partial_{3}\right),
\end{aligned}
$$

where $\nabla_{x}=\left(\partial_{1}, \partial_{2}, \partial_{3}\right)$. Here the symbols "." and " $\times$ " denote the inner and exterior products in $\mathbb{R}^{3}$, respectively. We put

$$
\Gamma=\left(\Gamma_{0}, \Gamma_{1}, \ldots, \Gamma_{10}\right)=(S, L, \Omega, \partial)=\left(S,\left(L_{j}\right)_{1 \leq j \leq 3},\left(\Omega_{j}\right)_{1 \leq j \leq 3},\left(\partial_{a}\right)_{0 \leq a \leq 3}\right),
$$

and we write $\Gamma^{\alpha}=\Gamma_{0}^{\alpha_{0}} \Gamma_{1}^{\alpha_{1}} \cdots \Gamma_{10}^{\alpha_{10}}$ with a multi-index $\alpha=\left(\alpha_{0}, \alpha_{1}, \ldots, \alpha_{10}\right)$. For a nonnegative integer $s$ and a smooth function $\varphi=\varphi(t, x)$, we define

$$
|\varphi(t, x)|_{s}=\sum_{|\alpha| \leq s}\left|\Gamma^{\alpha} \varphi(t, x)\right| \text {, and }\|\varphi(t, \cdot)\|_{s}=\left(\sum_{|\alpha| \leq s}\left\|\Gamma^{\alpha} \varphi(t, \cdot)\right\|_{L^{2}\left(\mathbb{R}^{3}\right)}^{2}\right)^{1 / 2} .
$$

For $(\varphi, \psi) \in \dot{H}^{1}\left(\mathbb{R}^{3}\right) \times L^{2}\left(\mathbb{R}^{3}\right)$, we define

$$
\mathcal{U}_{0}[\varphi, \psi](t, x):=u_{0}(t, x),
$$

where $u_{0}$ is the solution to (1.10)-(1.11) with

$$
\left(u_{0}, \partial_{t} u_{0}\right) \in C\left([0, \infty) ; \dot{H}^{1}\left(\mathbb{R}^{3}\right)\right) \times C\left([0, \infty) ; L^{2}\left(\mathbb{R}^{3}\right)\right) .
$$

Here $\dot{H}^{1}\left(\mathbb{R}^{3}\right)$ denotes the homogeneous Sobolev space which is the completion of $C_{0}^{\infty}\left(\mathbb{R}^{3}\right)$ with respect to the norm $\|\varphi\|_{\dot{H}^{1}\left(\mathbb{R}^{3}\right)}=\left\|\nabla_{x} \varphi\right\|_{L^{2}\left(\mathbb{R}^{3}\right)}$.

For $z \in \mathbb{R}^{d}$ with a positive integer $d$, the notation $\langle z\rangle=\sqrt{1+|z|^{2}}$ will be used throughout this paper. As in the introduction, we always put

$$
\omega_{0}=-1
$$

in what follows.

For the later convenience we allow $f$ and $g$ in (1.2) to depend on $\varepsilon$. More precisely, let $\mathcal{X}_{N}$ be the set of all mappings

$$
(f, g):[0,1] \ni \varepsilon \mapsto(f(\cdot ; \varepsilon), g(\cdot ; \varepsilon)) \in C_{0}^{\infty}\left(\mathbb{R}^{3} ; \mathbb{R}^{N}\right) \times C_{0}^{\infty}\left(\mathbb{R}^{3} ; \mathbb{R}^{N}\right)
$$

having the following two properties:

(1) There is some $R>0$, depending on $(f, g)$, such that $(f(x ; \varepsilon), g(x ; \varepsilon))=$ $(0,0)$ for $|x| \geq R$ and $\varepsilon \in[0,1]$.

(2) For any nonnegative integer $s$, we have

$$
\sup _{\varepsilon \in(0,1], x \in \mathbb{R}^{3}} \varepsilon^{-1} \sum_{|\alpha| \leq s}\left(\left|\partial_{x}^{\alpha}(f(x ; \varepsilon)-f(x ; 0))\right|+\left|\partial_{x}^{\alpha}(g(x ; \varepsilon)-g(x ; 0))\right|\right)<\infty .
$$


Here $\partial_{x}=\left(\partial_{1}, \partial_{2}, \partial_{3}\right)$, and we have used the standard notation of multi-indices. We replace the initial condition (1.2) with

$$
u(0, x)=\varepsilon f(x ; \varepsilon),\left(\partial_{t} u\right)(0, x)=\varepsilon g(x ; \varepsilon) \quad \text { for } x \in \mathbb{R}^{3},
$$

where $(f, g) \in \mathcal{X}_{N}$, and $\varepsilon$ is positive and small.

2.2. Basic assumption and examples. To state the main condition for our results, we introduce the following equivalence relation, which is motivated by the enhanced decay estimate for the null forms (see Lemma 3.3 below):

Definition 2.1. Let $F$ in (1.1) be given, and let $D \subset \mathcal{X}_{N}$.

For $\Phi(\omega, u, \partial u)$ and $\Psi(\omega, u, \partial u)$, which are homogeneous polynomials of degree 2 in $(u, \partial u)$ with smooth coefficients depending on $\omega \in S^{2}$, we write

$$
\Phi(\omega, u, \partial u) \stackrel{D}{\sim} \Psi(\omega, u, \partial u)
$$

if for any nonnegative integer $s$ there exists a positive constant $C_{s}$ such that the following property holds: If $u=u(t, x)$ satisfies (1.1)-(2.8) for $0 \leq t<T$ with some $(f, g) \in D, \varepsilon \in(0,1]$, and $T>0$, then it holds that

$$
\begin{aligned}
& \left|\Phi\left(|x|^{-1} x, u(t, x), \partial u(t, x)\right)-\Psi\left(|x|^{-1} x, u(t, x), \partial u(t, x)\right)\right|_{s} \\
& \quad \leq C_{s}\langle t+|x|\rangle^{-1}\left(|u(t, x)|_{[s / 2]+1}|\partial u(t, x)|_{s}+|\partial u(t, x)|_{[s / 2]}|u(t, x)|_{s+1}\right)
\end{aligned}
$$

for any $(t, x) \in[0, T) \times \mathbb{R}^{3}$ satisfying $|x| \geq t / 2 \geq 1$.

Now, motivated by (1.24), we introduce the following condition which we put on the nonlinearity $F(u, \partial u)=\left(F_{j}(u, \partial u)\right)_{1 \leq j \leq N}^{\mathrm{T}}$ in our theorems:

Condition 2.2. Setting

$$
\begin{aligned}
\left(v^{\mathrm{T}}, w^{\mathrm{T}}\right) & =\left(v_{1}, \ldots, v_{N^{\prime}}, w_{1}, \ldots, w_{N^{\prime \prime}}\right) \\
& :=\left(u_{1}, \ldots, u_{N^{\prime}}, u_{N^{\prime}+1}, \ldots u_{N}\right)=u^{\mathrm{T}}
\end{aligned}
$$

with $N^{\prime}+N^{\prime \prime}=N$, we can write $F$ as

$$
F_{j}(u, \partial u)= \begin{cases}F_{j}^{1}(\partial u)+F_{j}^{2}(u, \partial u), & 1 \leq j \leq N^{\prime}, \\ F_{j}^{1}(\partial u), & N^{\prime}+1 \leq j \leq N,\end{cases}
$$

where each $F_{j}^{1}$ is a homogeneous polynomial of degree 2 in $\partial u$, and

$$
F_{j}^{2}(u, \partial u)=\sum_{a=0}^{3} \sum_{k=1}^{N^{\prime}} \sum_{l=1}^{N^{\prime \prime}} p_{j}^{k a, l} w_{l}\left(\partial_{a} v_{k}\right)
$$

with some constants $p_{j}^{k a, l}$; furthermore, there exist some subset $D$ of $\mathcal{X}_{N}$, and some homogeneous polynomials $G_{j}(\omega, u, \partial u)\left(1 \leq j \leq N^{\prime}\right)$ of degree 2 in $(u, \partial u)$, which have the form

$$
G_{j}(\omega, u, \partial u)=\sum_{a=0}^{3} \sum_{k=1}^{N^{\prime}} \sum_{l=1}^{N^{\prime \prime}}\left(c_{j k}^{a, l}(\omega) w_{l}+\sum_{b=0}^{3} d_{j k}^{a, l b}(\omega)\left(\partial_{b} w_{l}\right)\right)\left(\partial_{a} v_{k}\right)
$$


with smooth coefficients $c_{j k}^{a, l}$ and $d_{j k}^{a, l b}$ on $S^{2}$, such that

$$
F_{j}(u, \partial u) \stackrel{D}{\sim} \begin{cases}G_{j}(\omega, u, \partial u), & 1 \leq j \leq N^{\prime} \\ 0, & N^{\prime}+1 \leq j \leq N\end{cases}
$$

It may seem difficult to check Condition 2.2 because it contains the relation given by the inequality (2.9). Here we give two kinds of algebraic conditions to ensure Condition 2.2.

Proposition 2.3. (1) Let $u=\left(u_{1}, \ldots, u_{N}\right)^{\mathrm{T}}$, and let $v$ and $w$ be given by (2.10). Suppose that $F=\left(F_{j}\right)_{1 \leq j \leq N}$ has the form

$$
F_{j}(u, \partial u)= \begin{cases}F_{j}^{0}(\partial u)+\widetilde{G}_{j}(u, \partial u), & 1 \leq j \leq N^{\prime} \\ F_{j}^{0}(\partial u), & N^{\prime}+1 \leq j \leq N\end{cases}
$$

where $\widetilde{G}_{j}$ is given by

$$
\widetilde{G}_{j}(u, \partial u)=\sum_{a=0}^{3} \sum_{k=1}^{N^{\prime}} \sum_{l=1}^{N^{\prime \prime}}\left(\widetilde{c}_{j k}^{a, l} w_{l}+\sum_{b=0}^{3} \widetilde{d}_{j k}^{a, l b}\left(\partial_{b} w_{l}\right)\right)\left(\partial_{a} v_{k}\right), \quad 1 \leq j \leq N^{\prime}
$$

with some constants $\widetilde{c}_{j k}^{a, l}$ and $\widetilde{d}_{j k}^{a, l b}$, while $F^{0}=\left(F_{j}^{0}\right)_{1 \leq j \leq N}^{\mathrm{T}}$ satisfies the null condition. Then Condition 2.2 is satisfied with $D=\mathcal{X}_{N}$ and $G_{j}(\omega, u, \partial u)=\widetilde{G}_{j}(u, \partial u)$ for $1 \leq j \leq N^{\prime}$.

(2) Let $F_{j}=F_{j}(\partial u)$ be a homogeneous polynomial of degree 2 in its arguments for $1 \leq j \leq N$. Then the system (1.1) satisfying Condition 1.1 with the initial condition (2.8) for $(f, g) \in \mathcal{X}_{N}$ can be reduced to another system of some size $N^{*}(\geq N)$, for which Condition 2.2 is satisfied with appropriately chosen $D\left(\subset \mathcal{X}_{N^{*}}\right)$.

We will prove Proposition 2.3 in Section 4. Note that the null condition implies Condition 2.2 with $D=\mathcal{X}_{N}$, because (2.14) holds with $\widetilde{G}_{j} \equiv 0$.

Remark 2.4. Our condition can be slightly weakened: We can add such terms as $w_{l}\left(\partial_{a} w_{m}\right)$ to $F_{j}^{2}$ for $1 \leq j \leq N^{\prime}$ in (2.11), and such ones as $w_{l}\left(\partial_{a} w_{m}\right)$ and $\left(\partial_{b} w_{l}\right)\left(\partial_{a} w_{m}\right)$, with smooth coefficients on $S^{2}$, to $G_{j}$ for $1 \leq j \leq N^{\prime}$ in (2.12) (here $l$ and $m$ run from 1 to $N^{\prime \prime}$; $a$ and $b$ from 0 to 3 ). These terms have been omitted just for simplicity of exposition. Indeed, in order to treat them, we only need to duplicate the equations for $w$, add them to the original system for $u$, and regard original $u$ and duplicated $w$ as new $v$ and $w$, respectively (namely we put $v_{N^{\prime}+m}=w_{m}$ for $1 \leq m \leq N^{\prime \prime}$, so that we have $w_{l}\left(\partial_{a} w_{m}\right)=w_{l}\left(\partial_{a} v_{N^{\prime}+m}\right)$ and so on $)$; we can see that Condition 2.2 is satisfied for this extended system with $N+N^{\prime \prime}$ components. The same is true for (1) of Proposition 2.3. 
2.3. Global existence. We define $\mathbb{R}_{+}:=[0, \infty)$. Concerning the existence of global solutions, we have the following:

Theorem 2.5. We fix a positive integer $m \geq 5$. We also fix two positive constants $\lambda$ and $\rho$ satisfying $0<\lambda<1 / 20$ and $1 / 2<\rho \leq 1-6 \lambda$. Suppose that Condition 2.2 is fulfilled. Let $D\left(\subset \mathcal{X}_{N}\right)$ be from Condition 2.2 . If $(f, g) \in D$, then there exists a positive constant $\varepsilon_{0}(\leq 1)$ such that for every $\varepsilon \in\left(0, \varepsilon_{0}\right]$ the Cauchy problem (1.1) (2.8) admits a unique global solution $u \in C^{\infty}\left(\mathbb{R}_{+} \times \mathbb{R}^{3} ; \mathbb{R}^{N}\right)$. Moreover there exists a positive constant $C$ such that

$$
\begin{aligned}
& \sup _{(t, x) \in \mathbb{R}_{+} \times \mathbb{R}^{3}}\langle t+|x|\rangle\left\{\langle t+|x|\rangle^{-\lambda}|v(t, x)|_{m+1}\right. \\
& \left.+\langle t-|x|\rangle^{\rho}|w(t, x)|_{m+2}\right\} \leq C \varepsilon, \\
& \sup _{t \in \mathbb{R}_{+}}\left\{(1+t)^{-\lambda}\|\partial v(t, \cdot)\|_{2 m}+\|\partial w(t, \cdot)\|_{2 m}\right\} \leq C \varepsilon
\end{aligned}
$$

for $\varepsilon \in\left(0, \varepsilon_{0}\right]$, where $v=\left(v_{1}, \ldots, v_{N^{\prime}}\right)^{\mathrm{T}}$ and $w=\left(w_{1}, \ldots, w_{N^{\prime \prime}}\right)^{\mathrm{T}}$ are given by (2.10).

If we put

$$
\widetilde{v}_{j}=v_{j}-\varepsilon \mathcal{U}_{0}\left[f_{j}, g_{j}\right] \text { and } \widetilde{w}_{k}=w_{k}-\varepsilon \mathcal{U}_{0}\left[f_{N^{\prime}+k}, g_{N^{\prime}+k}\right]
$$

for $1 \leq j \leq N^{\prime}$ and $1 \leq k \leq N^{\prime \prime}$, then we also have

$$
\begin{aligned}
& \sup _{(t, x) \in \mathbb{R}_{+} \times \mathbb{R}^{3}}\langle t+|x|\rangle\left\{\langle t+|x|\rangle^{-\lambda}|\widetilde{v}(t, x)|_{m+1}\right. \\
& \left.\quad+\langle t-|x|\rangle^{\rho}|\widetilde{w}(t, x)|_{m+2}\right\} \leq C \varepsilon^{2}, \\
& \sup _{t \in \mathbb{R}_{+}}\left\{(1+t)^{-\lambda}\|\partial \widetilde{v}(t, \cdot)\|_{2 m}+\|\partial \widetilde{w}(t, \cdot)\|_{2 m}\right\} \leq C \varepsilon^{2} .
\end{aligned}
$$

Here $\mathcal{U}_{0}\left[f_{j}, g_{j}\right]$ means $\mathcal{U}_{0}\left[f_{j}(\cdot ; \varepsilon), g_{j}(\cdot ; \varepsilon)\right]$ for $1 \leq j \leq N$.

This result can be proved by some modification of the arguments in Alinhac [3] (see also [12]). We will give the proof of Theorem 2.5 in Section 5. The estimates (2.15) - (2.18) play important roles in the proof of our next theorem on the pointwise behavior of the solutions.

2.4. Asymptotic pointwise behavior. Suppose that Condition 2.2 is fulfilled. Let an $\mathbb{R}^{N^{\prime \prime}}$-valued function

$$
\zeta=\zeta(\sigma, \omega)=\left(\zeta_{1}(\sigma, \omega), \ldots, \zeta_{N^{\prime \prime}}(\sigma, \omega)\right)^{\mathrm{T}}
$$

of $(\sigma, \omega) \in \mathbb{R} \times S^{2}$ be given. We define an $N^{\prime} \times N^{\prime}$ matrix-valued function $\mathbf{A}[\zeta]$ by

$$
\begin{aligned}
\mathbf{A}[\zeta](\sigma, \omega) & =\left(A_{j k}[\zeta](\sigma, \omega)\right)_{1 \leq j, k \leq N^{\prime}} \\
A_{j k}[\zeta](\sigma, \omega) & =-\frac{1}{2} \sum_{a=0}^{3} \omega_{a} \sum_{l=1}^{N^{\prime \prime}}\left(c_{j k}^{a, l}(\omega) \zeta_{l}(\sigma, \omega)+\sum_{b=0}^{3} d_{j k}^{a, l b}(\omega) \omega_{b}\left(\partial_{\sigma} \zeta_{l}\right)(\sigma, \omega)\right)
\end{aligned}
$$


for $(\sigma, \omega) \in \mathbb{R} \times S^{2}$, where the functions $c_{j k}^{a, l}$ and $d_{j k}^{a, l b}$ are from (2.12) in Condition 2.2. For a matrix $\mathbf{B}$ we define $e^{\mathbf{B}}(=\exp \mathbf{B})$ in the standard way of

$$
e^{\mathbf{B}}=\mathbf{I}+\sum_{k=1}^{\infty} \frac{1}{k !} \mathbf{B}^{k},
$$

where $\mathbf{I}$ is the identity matrix.

Now we are in a position to state our main result on the asymptotic pointwise behavior:

Theorem 2.6. Assume that Condition 2.2 is fulfilled, and let $\lambda, \rho$ and $\varepsilon_{0}$ be from Theorem 2.5. Let $D\left(\subset \mathcal{X}_{N}\right)$ be from Condition 2.2.

Suppose that we have $0<\varepsilon \leq \varepsilon_{0}$. Let $u=\left(v^{\mathrm{T}}, w^{\mathrm{T}}\right)^{\mathrm{T}}$ be the global solution to (1.1) -(2.8) with $(f, g) \in D$. Then there exist $V=V(\sigma, \omega)=\left(V_{j}(\sigma, \omega)\right)_{1 \leq j \leq N^{\prime}}^{\mathrm{T}}$, $W=W(\sigma, \omega)=\left(W_{k}(\sigma, \omega)\right)_{1 \leq k \leq N^{\prime \prime}}^{\mathrm{T}}$, and a positive constant $C$ such that we have

$$
\begin{aligned}
& \sum_{a=0}^{3}\left|r\left(\partial_{a} v\right)(t, r \omega)-\varepsilon \omega_{a} e^{(\varepsilon \log t) \mathbf{A}[W](r-t, \omega)}\left(\partial_{\sigma} V\right)(r-t, \omega)\right| \\
& \leq C \varepsilon\langle t+r\rangle^{3 \lambda+C \varepsilon-1}, \\
& |r w(t, r \omega)-\varepsilon W(r-t, \omega)| \leq C \varepsilon\langle t+r\rangle^{2 \lambda-1}\langle t-r\rangle^{1-\rho}, \\
& \sum_{a=0}^{3}\left|r\left(\partial_{a} w\right)(t, r \omega)-\varepsilon \omega_{a}\left(\partial_{\sigma} W\right)(r-t, \omega)\right| \leq C \varepsilon\langle t+r\rangle^{2 \lambda-1}\langle t-r\rangle^{-\rho}
\end{aligned}
$$

for any $(t, r) \in \mathbb{R}_{+} \times \mathbb{R}_{+}$with $r \geq t / 2 \geq 1$, and any $\omega=\left(\omega_{1}, \omega_{2}, \omega_{3}\right) \in S^{2}$, where $\mathbf{A}[W](\sigma, \omega)$ is given by (2.19). Here $V$ and $W$ may depend on $\varepsilon$, but the constant $C$ is independent of $\varepsilon$.

Moreover, there exists a positive constant $C$, being independent of $\varepsilon$, such that we have

$$
\begin{aligned}
& \sum_{j=1}^{N^{\prime}}\left|\partial_{\sigma} V_{j}(\sigma, \omega)-\partial_{\sigma} \mathcal{F}_{0}\left[f_{j}, g_{j}\right](\sigma, \omega)\right| \leq C \varepsilon(1+|\sigma|)^{3 \lambda+C \varepsilon-1} \\
& \sum_{k=1}^{N^{\prime \prime}}\left|\partial_{\sigma}^{l} W_{k}(\sigma, \omega)-\partial_{\sigma}^{l} \mathcal{F}_{0}\left[f_{k+N^{\prime}}, g_{k+N^{\prime}}\right](\sigma, \omega)\right| \leq C \varepsilon(1+|\sigma|)^{-\rho-l}
\end{aligned}
$$

for any $(\sigma, \omega) \in \mathbb{R} \times S^{2}$ and $l=0,1$, where $\mathcal{F}_{0}$ is defined by (1.13). Here $\mathcal{F}_{0}\left[f_{j}, g_{j}\right]$ means $\mathcal{F}_{0}\left[f_{j}(\cdot ; \varepsilon), g_{j}(\cdot ; \varepsilon)\right]$ for $1 \leq j \leq N$.

In what follows, we refer to $V$ in the above as the modified asymptotic profile for $v$, and $W$ as the standard asymptotic profile for $w$.

Remark 2.7. (i) Let $(\varphi, \psi) \in C_{0}^{\infty} \times C_{0}^{\infty}$, and suppose that $(\varphi, \psi) \not \equiv(0,0)$. Then we have $\partial_{\sigma} \mathcal{F}_{0}[\varphi, \psi] \not \equiv 0$ (see Section 3.3 below). Hence, for each $j \in\left\{1, \ldots, N^{\prime}\right\}$ 
(resp. $k \in\left\{1, \ldots, N^{\prime \prime}\right\}$ ), it follows from (2.24) (resp. (2.25)) that

$$
\left.\partial_{\sigma} V_{j} \not \equiv 0 \text { (resp. } \partial_{\sigma} W_{k} \not \equiv 0\right) \text { for } 0<\varepsilon \ll 1 \text {, }
$$

unless

$$
\left(f_{j}(\cdot ; 0), g_{j}(\cdot ; 0)\right) \equiv(0,0)\left(\operatorname{resp} .\left(f_{k+N^{\prime}}(\cdot ; 0), g_{k+N^{\prime}}(\cdot ; 0)\right) \equiv(0,0)\right)
$$

in Theorem 2.6.

(ii) If $\varphi, \psi \in C_{0}^{\infty}\left(\mathbb{R}^{3}\right)$, then $\mathcal{F}_{0}[\varphi, \psi](\sigma, \omega)=0$ for large $|\sigma|$ (see (3.22) below), and $\mathcal{F}_{0}[\varphi, \psi]$ is bounded in $\mathbb{R} \times S^{2}$. Therefore (2.24) and (2.25) yield

$$
\begin{aligned}
\left|\partial_{\sigma} V(\sigma, \omega)\right| & \leq C(1+|\sigma|)^{3 \lambda+C \varepsilon-1}, \\
\left|\partial_{\sigma}^{l} W(\sigma, \omega)\right| & \leq C(1+|\sigma|)^{-\rho-l}, \quad l=0,1
\end{aligned}
$$

for $(\sigma, \omega) \in \mathbb{R} \times S^{2}$ and $0<\varepsilon \leq \varepsilon_{0}$, respectively.

The asymptotic behavior in the energy sense also follows from Theorem 2.6 .

Corollary 2.8. Suppose that all the assumptions in Theorem 2.6 are fulfilled, and let $u=\left(v^{\mathrm{T}}, w^{\mathrm{T}}\right)^{\mathrm{T}}, W$, and $D$ be as in Theorem 2.6. Then, for $(f, g) \in D$ and sufficiently small $\varepsilon>0$, there exist some functions $\left(f_{j}^{+}, g_{j}^{+}\right) \in \dot{H}^{1}\left(\mathbb{R}^{3}\right) \times L^{2}\left(\mathbb{R}^{3}\right)$ $(j=1, \ldots, N)$ such that we have

$$
\begin{aligned}
& \lim _{t \rightarrow \infty}\left(\frac{1}{2} \sum_{a=0}^{3}\left\|e^{-\Theta_{\varepsilon}^{+}(t, \cdot)} \partial_{a} v(t, \cdot)-\partial_{a} v^{+}(t, \cdot)\right\|_{L^{2}\left(\mathbb{R}^{3}\right)}^{2}\right)^{1 / 2}=0 \\
& \lim _{t \rightarrow \infty}\left\|w(t, \cdot)-w^{+}(t, \cdot)\right\|_{E}=0
\end{aligned}
$$

where

$$
\begin{aligned}
\left(\left(v^{+}\right)^{\mathrm{T}},\left(w^{+}\right)^{\mathrm{T}}\right) & =\left(v_{1}^{+}, \ldots, v_{N^{\prime}}^{+}, w_{1}^{+}, \ldots, w_{N^{\prime \prime}}^{+}\right)=\left(\varepsilon \mathcal{U}_{0}\left[f_{1}^{+}, g_{1}^{+}\right], \ldots, \varepsilon \mathcal{U}_{0}\left[f_{N}^{+}, g_{N}^{+}\right]\right), \\
\boldsymbol{\Theta}_{\varepsilon}^{+}(t, x) & = \begin{cases}(\varepsilon \log t) \mathbf{A}[W](|x|-t, x /|x|), & (t, x) \in[2, \infty) \times\left(\mathbb{R}^{3} \backslash\{0\}\right), \\
\mathbf{O}, & \text { otherwise } .\end{cases}
\end{aligned}
$$

Here $\|\cdot\|_{E}$ is given by (1.8), and $\mathbf{O}$ is the zero matrix.

Theorem 2.6 and Corollary 2.8 will be proved in Sections 6] and 7, respectively.

Comparing (2.22) and (2.23) with (1.15), we see that $w$ behaves similarly to the free solutions in the pointwise sense (and (2.29) says that $w$ is asymptotically free in the energy sense), but $v$ may behave quite differently from the free solutions because of the exponential factor in (2.21) (and also in (2.28) ). Here we give some applications of Theorem 2.6 to answer Question 1.3 in the introduction. To simplify the exposition, we introduce the following notation: For functions $\varphi=\varphi(t, r, \omega)$ and $\psi=\psi(t, r, \omega)$ of $(t, r, \omega) \in \mathbb{R}_{+} \times \mathbb{R}_{+} \times S^{2}$, we write $\varphi(t, r, \omega) \approx \psi(t, r, \omega)$, if we have

$$
\lim _{t \rightarrow \infty}|(\varphi-\psi)(t, t+\sigma, \omega)|=0, \quad(\sigma, \omega) \in \mathbb{R} \times S^{2}
$$


Example 2.9. Let $\left(u_{1}, u_{2}, w\right)^{\mathrm{T}}$ be the global solution to (1.24) with initial data

$$
\left(u_{1}, u_{2}, w\right)^{\mathrm{T}}=\varepsilon\left(f_{1}, f_{2}, f_{3}\right)^{\mathrm{T}},\left(\partial_{t} u_{1}, \partial_{t} u_{2}, \partial_{t} w\right)^{\mathrm{T}}=\varepsilon\left(g_{1}, g_{2}, g_{3}\right)^{\mathrm{T}} \text { at } t=0,
$$

and set $v=\left(v_{1}, v_{2}\right)^{\mathrm{T}}=\left(u_{1}, u_{2}\right)^{\mathrm{T}}$, where $\varepsilon$ is assumed to be sufficiently small. We suppose $(f, g) \in \mathcal{X}_{3}$ with $f=\left(f_{j}\right)_{1 \leq j \leq 3}^{\mathrm{T}}$ and $g=\left(g_{j}\right)_{1 \leq j \leq 3}^{\mathrm{T}}$. Note that if we want to treat (1.23), then we only have to impose restrictions

$$
f_{3}=\partial_{1} f_{2}-\partial_{2} f_{1}, g_{3}=\partial_{1} g_{2}-\partial_{2} g_{1} .
$$

Concerning (1.24), we get

$$
\mathbf{A}[\zeta](\sigma, \omega)=-\frac{\zeta(\sigma, \omega)}{2}\left(\begin{array}{ll}
\omega_{1} & 0 \\
\omega_{2} & 0
\end{array}\right), \text { and } e^{\tau \mathbf{A}[\zeta](\sigma, \omega)}=\left(\begin{array}{cc}
e^{-\tau \omega_{1} \zeta(\sigma, \omega) / 2} & 0 \\
\frac{\omega_{2}}{\omega_{1}}\left(e^{-\tau \omega_{1} \zeta(\sigma, \omega) / 2}-1\right) & 1
\end{array}\right)
$$

for $(\sigma, \omega) \in \mathbb{R} \times S^{2}$ and $\tau \in \mathbb{R}$, where $\left.\left(\left(\omega_{2} / \omega_{1}\right)\left(e^{-\tau \omega_{1} \zeta(\sigma, \omega) / 2}-1\right)\right)\right|_{\omega_{1}=0}$ is regarded as $-\left.2^{-1} \tau \omega_{2} \zeta(\sigma, \omega)\right|_{\omega_{1}=0}$. By Theorem [2.6, there exist an $\mathbb{R}^{2}$-valued function $V=$ $V(\sigma, \omega)=\left(V_{1}(\sigma, \omega), V_{2}(\sigma, \omega)\right)^{\mathrm{T}}$ and a real-valued function $W=W(\sigma, \omega)$ such that

$$
\begin{aligned}
r\left(\partial_{a} v_{1}\right)(t, r \omega) \approx & \varepsilon \omega_{a} t^{-\varepsilon \omega_{1} W(r-t, \omega) / 2}\left(\partial_{\sigma} V_{1}\right)(r-t, \omega), \\
r\left(\partial_{a} v_{2}\right)(t, r \omega) \approx & \varepsilon \omega_{a} \frac{\omega_{2}}{\omega_{1}}\left(t^{-\varepsilon \omega_{1} W(r-t, \omega) / 2}-1\right)\left(\partial_{\sigma} V_{1}\right)(r-t, \omega) \\
& +\varepsilon \omega_{a}\left(\partial_{\sigma} V_{2}\right)(r-t, \omega), \\
r w(t, r \omega) \approx & \varepsilon W(r-t, \omega), \quad r\left(\partial_{a} w\right)(t, r \omega) \approx \varepsilon \omega_{a}\left(\partial_{\sigma} W\right)(r-t, \omega)
\end{aligned}
$$

for $0 \leq a \leq 3$. From this asymptotic pointwise behavior, we find that $\partial v_{1}$ decays slower than the free solutions along the line $\{(t,(t+\sigma) \omega) ; t>0\}$ for fixed $(\sigma, \omega) \in$ $\mathbb{R} \times S^{2}$ if $\omega_{1} W(\sigma, \omega)<0$, and faster if $\omega_{1} W(\sigma, \omega)>0$. We can also recover the previous result in [12] from (2.32)-(2.34); namely we can show that if we choose appropriate initial profile (satisfying (2.31) ), then we have

$$
C_{1} \varepsilon(1+t)^{C_{1} \varepsilon} \leq\|u(t)\|_{E} \leq C_{2} \varepsilon(1+t)^{C_{2} \varepsilon}
$$

with some positive constants $C_{1}$ and $C_{2}$. (2.35), as well as (2.39) and (2.42) below, will be proved in Proposition 7.4 .

Example 2.10. Let $u=\left(u_{1}, u_{2}, u_{3}\right)^{\mathrm{T}}=\left(v_{1}, v_{2}, w\right)^{\mathrm{T}}$, and consider

$$
\left\{\begin{array}{l}
\square v_{1}=-2\left(\partial_{t} w\right)\left(\partial_{t} v_{2}\right)+F_{1}^{0}(\partial u), \\
\square v_{2}=F_{2}^{0}(\partial u), \\
\square w=F_{3}^{0}(\partial u)
\end{array}\right.
$$

in $(0, \infty) \times \mathbb{R}^{3}$ with the initial condition (2.8) for small $\varepsilon$, where $F^{0}=\left(F_{j}^{0}\right)_{1 \leq j \leq 3}^{\mathrm{T}}$ satisfies the null condition (note that a similar and simpler example is mentioned in [21] and [22] as an example for the weak null condition). Then we have

$$
\mathbf{A}[\zeta](\sigma, \omega)=\left(\begin{array}{cc}
0 & \left(\partial_{\sigma} \zeta\right)(\sigma, \omega) \\
0 & 0
\end{array}\right), \text { and } e^{\tau \mathbf{A}[\zeta](\sigma, \omega)}=\left(\begin{array}{cc}
1 & \tau\left(\partial_{\sigma} \zeta\right)(\sigma, \omega) \\
0 & 1
\end{array}\right)
$$


for $(\sigma, \omega) \in \mathbb{R} \times S^{2}$ and $\tau \in \mathbb{R}$. By Theorem 2.6, for any $(f, g) \in \mathcal{X}_{3}$ there exist $V=V(\sigma, \omega)$ and $W=W(\sigma, \omega)$ such that we have (2.34) and

$$
\begin{aligned}
r\left(\partial_{a} v_{1}\right)(t, r \omega) \approx & \varepsilon \omega_{a}\left(\partial_{\sigma} V_{1}\right)(r-t, \omega) \\
& +\varepsilon^{2} \omega_{a}(\log t)\left(\partial_{\sigma} W\right)(r-t, \omega)\left(\partial_{\sigma} V_{2}\right)(r-t, \omega), \\
r\left(\partial_{a} v_{2}\right)(t, r \omega) \approx & \varepsilon \omega_{a}\left(\partial_{\sigma} V_{2}\right)(r-t, \omega)
\end{aligned}
$$

for $0 \leq a \leq 3$. This asymptotic pointwise behavior results in the slower growth of the energy than (2.35); if we choose appropriate initial profile, then we have

$$
C_{1}\left(\varepsilon+\varepsilon^{2} \log (1+t)\right) \leq\|u(t)\|_{E} \leq C_{2}\left(\varepsilon+\varepsilon^{2} \log (1+t)\right)
$$

with some positive constants $C_{1}$ and $C_{2}$. We remark that this kind of logarithmic growth of the energy was observed by Sunagawa [23] for a system of semilinear Klein-Gordon equations with different masses (see also [24] for the pointwise behavior).

Example 2.11. Let $u=\left(u_{1}, u_{2}, u_{3}\right)^{\mathrm{T}}=\left(v_{1}, v_{2}, w\right)^{\mathrm{T}}$, and we consider

$$
\left\{\begin{array}{l}
\square v_{1}=-2\left(\partial_{t} w\right)\left(\partial_{t} v_{2}\right)+F_{1}^{0}(\partial u), \\
\square v_{2}=2\left(\partial_{t} w\right)\left(\partial_{t} v_{1}\right)+F_{2}^{0}(\partial u), \\
\square w=F_{3}^{0}(\partial u)
\end{array}\right.
$$

in $(0, \infty) \times \mathbb{R}^{3}$ with the initial condition (2.8), where $F^{0}=\left(F_{j}^{0}\right)_{1 \leq j \leq 3}^{\mathrm{T}}$ satisfies the null condition as before, and $\varepsilon$ is assumed to be sufficiently small. For this example, we have

$$
\mathbf{A}[\zeta](\sigma, \omega)=\left(\partial_{\sigma} \zeta\right)(\sigma, \omega)\left(\begin{array}{cc}
0 & 1 \\
-1 & 0
\end{array}\right)
$$

Hence, by Theorem 2.6, for any $(f, g) \in \mathcal{X}_{3}$ there exist $V=V(\sigma, \omega)$ and $W=$ $W(\sigma, \omega)$ such that we have (2.34) and

$$
\left(\begin{array}{l}
r\left(\partial_{a} v_{1}\right)(t, r \omega) \\
r\left(\partial_{a} v_{2}\right)(t, r \omega)
\end{array}\right) \approx \varepsilon \omega_{a} e^{(\varepsilon \log t) \mathbf{A}[W](r-t, \omega)}\left(\begin{array}{c}
\left(\partial_{\sigma} V_{1}\right)(r-t, \omega) \\
\left(\partial_{\sigma} V_{2}\right)(r-t, \omega)
\end{array}\right)
$$

for $0 \leq a \leq 3$, where

$$
e^{\tau \mathbf{A}[W](\sigma, \omega)}=\left(\begin{array}{cc}
\cos \left(\tau\left(\partial_{\sigma} W\right)(\sigma, \omega)\right) & \sin \left(\tau\left(\partial_{\sigma} W\right)(\sigma, \omega)\right) \\
-\sin \left(\tau\left(\partial_{\sigma} W\right)(\sigma, \omega)\right) & \cos \left(\tau\left(\partial_{\sigma} W\right)(\sigma, \omega)\right)
\end{array}\right) .
$$

There is no growth or decay factor in $e^{(\varepsilon \log t) \mathbf{A}[W]}$, and we can show that

$$
C_{1} \varepsilon \leq\|u(t)\|_{E} \leq C_{2} \varepsilon
$$

for small $\varepsilon>0$ with some positive constants $C_{1}$ and $C_{2}$, unless $(f(\cdot ; 0), g(\cdot ; 0)) \equiv$ $(0,0)$. Thus the energy of this system stays bounded from above and below by positive constants for any initial profile not vanishing at $\varepsilon=0$ (actually the energy is preserved for any initial profile if $\left.F^{0} \equiv 0\right)$. However the solution behaves quite differently from the free solution in the pointwise sense, as far as $\left(\partial_{\sigma} W\right)(\sigma, \omega) \not \equiv 0$. Furthermore we can also show that there exists some initial profile $(f, g) \in \mathcal{X}_{3}$ such 
that the global solution to (2.40) is not asymptotically free in the energy sense for $0<\varepsilon \ll 1$ (see Proposition 7.4 below for the proof). This example gives a positive answer to Question 1.3.

Remark 2.12. Observe that Condition 1.1 is not satisfied for (2.40), while it is fulfilled for (1.23) and (2.36). This is the reason why we have introduced Condition 2.2 .

2.5. Asymptotically free solutions and the null condition. Our aim here is to answer Question 1.4 in the introduction.

Definition 2.13. We say that a global solution $u=u(t, x)$ to (1.1) is asymptotically free in the pointwise sense, if there exists some function $U=U(\sigma, \omega)$ such that $r \partial_{a} u(t, r \omega) \approx \omega_{a} \partial_{\sigma} U(r-t, \omega)$ for $0 \leq a \leq 3$, namely

$$
\lim _{t \rightarrow \infty} \sum_{a=0}^{3}\left|\left\{r \partial_{a} u(t, r \omega)-\omega_{a}\left(\partial_{\sigma} U\right)(r-t, \omega)\right\}\right|_{r=t+\sigma} \mid=0, \quad(\sigma, \omega) \in \mathbb{R} \times S^{2} .
$$

In view of (1.15), by choosing the Friedlander radiation field as $U$, we see that the solution to the free wave equation $\square u=0$ with $C_{0}^{\infty}$-data is asymptotically free in the pointwise sense.

We say that $(\mathrm{AFP})$ (resp. (AFE)) holds if for any $(f, g) \in \mathcal{X}_{N}$ there exists a positive constant $\varepsilon_{1}$ such that, for any $\varepsilon \in\left(0, \varepsilon_{1}\right]$, the global solution $u$ to the Cauchy problem (1.1)-(2.8) is asymptotically free in the pointwise sense (resp. in the energy sense).

Theorem 2.14. For the system (1.1) with each $F_{j}=F_{j}(u, \partial u)$ being a homogeneous polynomial of degree 2 in its arguments, the following two are equivalent:

(1) The null condition is satisfied.

(2) Condition 2.2 is satisfied with $D=\mathcal{X}_{N}$, and (AFP) holds.

We conjecture that (1) above is also equivalent to (2) with (AFP) being replaced by (AFE), but this conjecture is still open.

Now let us consider the case of Condition 1.1. Though we can rewrite the system to another system satisfying Condition 2.2 (see Proposition 2.3), we cannot apply Theorem 2.14 directly because of the restriction on initial data for the rewritten system. Thus we need to consider this case separately. Making use of the "rank one" structure (1.19), we can get a better result than Theorem 2.14.

Theorem 2.15. For the system (1.1) with each $F_{j}=F_{j}(\partial u)$ being a homogeneous polynomial of degree 2 in $\partial u$, the following three are equivalent to each other:

(1) The null condition is satisfied.

(2) Condition 1.1 is satisfied, and (AFP) holds.

(3) Condition 1.1 is satisfied, and (AFE) holds.

Theorems 2.14 and 2.15 will be proved in Section 8 . 


\section{Preliminaries}

3.1. Vector fields associated with wave equations. First we recall some properties of the vector fields contained in $\Gamma$ (remember the notation in Section 2.1). We have $[S, \square]=-2 \square$, and $\left[\Omega_{j}, \square\right]=\left[L_{j}, \square\right]=\left[\partial_{a}, \square\right]=0$ for $1 \leq j \leq 3$ and $0 \leq a \leq 3$, where $[A, B]=A B-B A$ for operators $A$ and $B$. Thus we get

$$
\square\left(\Gamma^{\alpha} \varphi\right)=\left(\Gamma_{0}+2\right)^{\alpha_{0}} \Gamma_{1}^{\alpha_{1}} \cdots \Gamma_{10}^{\alpha_{10}}(\square \varphi)
$$

for any smooth function $\varphi$ and any multi-index $\alpha=\left(\alpha_{0}, \alpha_{1}, \ldots, \alpha_{10}\right)$. Since $\left[\Gamma_{a}, \Gamma_{b}\right]$ with $0 \leq a, b \leq 10$ can be written in terms of $\Gamma$, it follows that

$$
\Gamma^{\alpha} \Gamma^{\beta}=\Gamma^{\alpha+\beta}+\sum_{|\gamma| \leq|\alpha|+|\beta|-1} c_{\gamma}^{\alpha, \beta} \Gamma^{\gamma}
$$

for any multi-indices $\alpha$ and $\beta$ with appropriate constants $c_{\gamma}^{\alpha, \beta}$. Especially $\left[\Gamma_{a}, \partial_{b}\right]$ for $0 \leq a \leq 10$ and $0 \leq b \leq 3$ can be written in terms of $\partial$. Therefore for any nonnegative integer $s$, there exists a positive constant $C_{s}$ such that

$$
C_{s}^{-1}|\partial \varphi(t, x)|_{s} \leq \sum_{|\alpha| \leq s} \sum_{a=0}^{3}\left|\partial_{a} \Gamma^{\alpha} \varphi(t, x)\right| \leq C_{s}|\partial \varphi(t, x)|_{s}
$$

for any sufficiently smooth function $\varphi=\varphi(t, x)$, where $|\cdot|_{s}$ is given by (2.6).

The following Sobolev type inequality, known as the Klainerman inequality, is quite useful in deriving decay estimates for wave equations (see Klainerman [17] for the proof; see also [15] and [7]).

Lemma 3.1. There exists a positive constant $C$ such that we have

$$
\sup _{x \in \mathbb{R}^{3}}\langle t+|x|\rangle\langle t-|x|\rangle^{1 / 2}|\varphi(t, x)| \leq C\|\varphi(t, \cdot)\|_{2}
$$

for any $C^{2}$-function $\varphi$, provided that the right-hand side of (3.4) is finite. Here $\|\cdot\|_{s}$ is given by (2.6).

We set $r=|x|, \omega=r^{-1} x$, and $\partial_{r}=\sum_{j=1}^{3}\left(x_{j} /|x|\right) \partial_{j}$. We also define $L_{r}=$ $r \partial_{t}+t \partial_{r}=\sum_{j=1}^{3}\left(x_{j} /|x|\right) L_{j}$, and $\partial_{ \pm}=\partial_{t} \pm \partial_{r}$. Since we have $S=t \partial_{t}+r \partial_{r}$, we obtain

$$
\partial_{+}=\frac{1}{t+r}\left(S+L_{r}\right)
$$

Note that (3.5) implies $\partial_{+}=(1+t+r)^{-1}\left(S+L_{r}+\partial_{t}+\partial_{r}\right)$. Since $\partial_{t}=\left(\partial_{-}+\partial_{+}\right) / 2$ and $\partial_{r}=\left(-\partial_{-}+\partial_{+}\right) / 2$, we obtain from (3.5) that

$$
\left|\left(\partial_{t}-\frac{1}{2} \partial_{-}\right) \varphi(t, x)\right|+\left|\left(\partial_{r}+\frac{1}{2} \partial_{-}\right) \varphi(t, x)\right| \leq C\langle t+r\rangle^{-1}|\varphi(t, x)|_{1}
$$

for any smooth function $\varphi$. Since

$$
\nabla_{x}=\omega \partial_{r}-r^{-1} \omega \times \Omega=\omega \partial_{r}-t^{-1} \omega \times(\omega \times L),
$$

we get

$$
\left|\left(\partial_{j}-\omega_{j} \partial_{r}\right) \varphi(t, x)\right| \leq C\langle t+r\rangle^{-1}|\varphi(t, x)|_{1}, \quad j=1,2,3 .
$$


Now (3.6) and (3.8) lead to the following estimate (3.9) and its immediate consequence $(3.10)$ :

Lemma 3.2. There exists a positive constant $C$ such that we have

$$
\begin{aligned}
& \sum_{a=0}^{3}\left|\left(\partial_{a}-\frac{-\omega_{a}}{2} \partial_{-}\right) \varphi(t, x)\right| \leq C\langle t+r\rangle^{-1}|\varphi(t, x)|_{1}, \\
& \sum_{a=0}^{3}\left|r\left(\partial_{a} \varphi\right)(t, x)-\frac{-\omega_{a}}{2} \partial_{-}(r \varphi(t, x))\right| \leq C|\varphi(t, x)|_{1}
\end{aligned}
$$

for any smooth function $\varphi=\varphi(t, x)$, where $\omega=\left(\omega_{1}, \omega_{2}, \omega_{3}\right)=|x|^{-1} x$, and $r=|x|$.

Recalling the definition of the null condition (1.3), we obtain from Lemma 3.2 the following estimate for the null forms given by (1.4) and (1.5) (see Klainerman [16] for the details of the proof).

Lemma 3.3. Let $Q$ be one of the null forms $Q_{0}$ and $Q_{a b}$ with $0 \leq a<b \leq 3$. Then, for any nonnegative integer $s$, there exists a positive constant $C_{s}$ such that

$$
\left|Q\left(u_{k}, u_{l}\right)\right|_{s} \leq C_{s}\langle t+|x|\rangle^{-1}\left(|u|_{[s / 2]+1}|\partial u|_{s}+|\partial u|_{[s / 2]}|u|_{s+1}\right), \quad 1 \leq k, l \leq N
$$

at $(t, x) \in(0, \infty) \times \mathbb{R}^{3}$ for any smooth function $u=u(t, x)=\left(u_{j}(t, x)\right)_{1 \leq j \leq N}^{\mathrm{T}}$, where $[m]$ denotes the largest integer not exceeding the number $m$.

Since $t S-r L_{r}=\left(t^{2}-r^{2}\right) \partial_{t}$ and $t L_{r}-r S=\left(t^{2}-r^{2}\right) \partial_{r}$, we get

$$
\langle t-r\rangle\left(\left|\partial_{t} \varphi(t, x)\right|+\left|\partial_{r} \varphi(t, x)\right|\right) \leq C|\varphi(t, x)|_{1} .
$$

Equations (3.8) and (3.11) yield the following (see also Lindblad [20]).

Lemma 3.4. There exists a positive constant $C$ such that

$$
\langle t-r\rangle|\partial \varphi(t, x)| \leq C|\varphi(t, x)|_{1}
$$

holds for any smooth function $\varphi=\varphi(t, x)$.

For $R>0$, we define

$$
B_{R}:=\left\{x \in \mathbb{R}^{3} ;|x| \leq R\right\} .
$$

The following Hardy type inequality is due to Lindblad [20].

Lemma 3.5. Let $R>0$ be given. Then there exists a positive constant $C_{R}$, which depends on $R$, such that

$$
\left(\int_{\mathbb{R}^{3}} \frac{|\varphi(t, x)|^{2}}{\langle t-|x|\rangle^{2}} d x\right)^{1 / 2} \leq C_{R}\|\partial \varphi(t, \cdot)\|_{L^{2}\left(\mathbb{R}^{3}\right)}, \quad t \geq 0
$$

holds for any smooth function $\varphi=\varphi(t, x)$ satisfying

$$
\operatorname{supp} \varphi(t, \cdot) \subset B_{t+R}, \quad t \geq 0 .
$$


Now we introduce

$$
Z=\left(Z_{1}, Z_{2}, Z_{3}\right):=\omega \partial_{t}+\nabla_{x}=\left(\omega_{j} \partial_{t}+\partial_{j}\right)_{1 \leq j \leq 3}
$$

where $\omega=\left(\omega_{1}, \omega_{2}, \omega_{3}\right)=|x|^{-1} x \in S^{2}$. For a nonnegative integer $s$ and a smooth function $\varphi=\varphi(t, x)$, we define

$$
|\varphi(t, x)|_{Z, s}=\sum_{j=1}^{3} \sum_{|\alpha| \leq s}\left|Z_{j} \Gamma^{\alpha} \varphi(t, x)\right| .
$$

Then we obtain the following inequality.

Lemma 3.6. For a nonnegative integer $s$, there exists a positive constant $C_{s}$ such that

$$
|\Gamma \varphi(t, x)|_{s} \leq C_{s}\left(r|\varphi(t, x)|_{Z, s}+\langle t-r\rangle|\partial \varphi(t, x)|_{s}\right)
$$

for any smooth function $\varphi=\varphi(t, x)$, where $\Gamma \varphi=\left(\Gamma_{a} \varphi\right)_{0 \leq a \leq 10}$.

Proof. It is easy to check that

$$
S=x \cdot Z+(t-r) \partial_{t}, L=r Z+(t-r) \nabla_{x}, \Omega=x \times Z
$$

Hence we get

$$
|\Gamma \varphi|_{s} \leq C \sum_{a=0}^{10} \sum_{|\beta| \leq s}\left|\Gamma_{a} \Gamma^{\beta} \varphi\right| \leq C r|\varphi|_{Z, s}+C\langle t-r\rangle \sum_{a=0}^{3} \sum_{|\beta| \leq s}\left|\partial_{a} \Gamma^{\beta} \varphi\right|,
$$

which implies the desired result because of (3.3).

By (3.7) and (3.5), we have

$$
Z=\omega \partial_{+}-r^{-1} \omega \times \Omega=\omega(t+r)^{-1}(S+\omega \cdot L)-r^{-1} \omega \times \Omega .
$$

By direct calculations, we have

$$
\left[\partial_{t}, Z_{j}\right]=0,\left[\partial_{k}, Z_{j}\right]=r^{-1}\left(\delta_{j k}-\omega_{j} \omega_{k}\right) \partial_{t}
$$

for $1 \leq j, k \leq 3$, where $\delta_{j k}$ is the Kronecker delta. For a nonnegative integer $s$, there is a positive constant $C_{s}$ such that

$$
\begin{aligned}
& \sum_{j=1}^{3}\left(\left|(t+r)^{-1} \omega_{j}\right|_{s}+\left|r^{-1} \omega_{j}\right|_{s}\right) \\
& \quad+\sum_{j, k=1}^{3}\left(\left|(t+r)^{-1} \omega_{j} \omega_{k}\right|_{s}+\left|r^{-1}\left(\delta_{j k}-\omega_{j} \omega_{k}\right)\right|_{s}\right) \leq C_{s}\langle t+r\rangle^{-1}
\end{aligned}
$$

when $r \geq t / 2 \geq 1$. Thus we obtain the following estimate. 
Lemma 3.7. For any positive integer $s$, there exists a positive constant $C_{s}$ such that

$$
\begin{gathered}
\sum_{j=1}^{3}\left|Z_{j} \varphi(t, x)\right|_{s} \leq C_{s}\langle t+r\rangle^{-1}|\varphi(t, x)|_{s+1}, \\
\sum_{a=0}^{3} \sum_{j=1}^{3}\left|\partial_{a} Z_{j} \varphi(t, x)\right|_{s} \leq C_{s}\langle t+r\rangle^{-1}|\partial \varphi(t, x)|_{s+1},
\end{gathered}
$$

for any smooth function $\varphi$ and any $(t, x)$ with $r \geq t / 2 \geq 1$.

3.2. Basic estimates for wave equations. Here we give two basic estimates for the solution to the inhomogeneous wave equation

$$
\square u(t, x)=F(t, x), \quad(t, x) \in(0, \infty) \times \mathbb{R}^{3}
$$

with initial data

$$
u(0, x)=\varphi(x),\left(\partial_{t} u\right)(0, x)=\psi(x), \quad x \in \mathbb{R}^{3} .
$$

We suppose that $\varphi, \psi$ and $F$ are smooth functions.

The following improved energy inequality is due to Alinhac [2] and LindbladRodnianski [22] (see also Alinhac [1] and [3]).

Lemma 3.8. Let $u$ be the solution to (3.17)-(3.18). Then, for $\mu \geq 0$ and $\rho>0$, there exists a positive constant $C=C(\rho)$ such that

$$
\begin{aligned}
\langle t\rangle^{-\mu}\|\partial u(t, \cdot)\|_{L^{2}}+\left(\int_{0}^{t} \int_{\mathbb{R}^{3}} \frac{|Z u(\tau, x)|^{2}}{\langle\tau\rangle^{2 \mu}\langle\tau-|x|\rangle^{1+\rho}} d x d \tau\right)^{1 / 2} \\
\leq C\left(\left\|\nabla_{x} \varphi\right\|_{L^{2}}+\|\psi\|_{L^{2}}+\int_{0}^{t}\langle\tau\rangle^{-\mu}\|F(\tau, \cdot)\|_{L^{2}} d \tau\right)
\end{aligned}
$$

for $t \geq 0$, where $Z$ is given by (3.13).

Outline of the proof. We multiply $\square u$ by $\langle t\rangle^{-2 \mu} e^{\zeta_{\rho}(|x|-t)}\left(\partial_{t} u\right)$, integrate it over $\mathbb{R}^{3}$, and perform the integration by parts as in the standard energy estimate, where

$$
\zeta_{\rho}(s)=\int_{-\infty}^{s}\langle\tau\rangle^{-(1+\rho)} d \tau
$$

Then we obtain the desired result, since there exists a positive constant $C_{\rho}$ such that $1 \leq e^{\zeta_{\rho}(s)} \leq C_{\rho}$ for $s \in \mathbb{R}$.

Now we turn our attention to the decay estimate. We define

$$
\mathcal{W}_{\rho}(t, r)= \begin{cases}\langle t+r\rangle^{\rho} & \text { if } \rho<0 \\ \left\{\log \left(2+\langle t+r\rangle\langle t-r\rangle^{-1}\right)\right\}^{-1} & \text { if } \rho=0 \\ \langle t-r\rangle^{\rho} & \text { if } \rho>0\end{cases}
$$


Lemma 3.9. Let $u$ be the solution to (3.17)-(3.18). Suppose that $\mu>0$ and $\kappa>1$. Then there exists a positive constant $C=C(\mu, \kappa)$ such that

$$
\begin{aligned}
& \langle t+|x|\rangle \mathcal{W}_{\mu-1}(t,|x|)|u(t, x)| \\
& \leq C \sup _{|y-x| \leq t}\langle y\rangle^{\mu}\left(\langle y\rangle \sum_{|\alpha| \leq 1}\left|\left(\partial_{x}^{\alpha} \varphi\right)(y)\right|+|y||\psi(y)|\right) \\
& \quad+C \sup _{\tau \in[0, t]} \sup _{|y-x| \leq t-\tau}|y|\langle\tau+|y|\rangle^{\mu}\langle\tau-|y|\rangle^{\kappa}|F(\tau, y)|
\end{aligned}
$$

for $(t, x) \in \mathbb{R}_{+} \times \mathbb{R}^{3}$, provided that the right-hand side of (3.19) is finite. Here $\partial_{x}=\left(\partial_{1}, \partial_{2}, \partial_{3}\right)$, and we have used the standard notation of multi-indices.

Outline of the proof. The case $F \equiv 0$ is essentially proved in Asakura 4, Proposition 1.1] (see also Katayama-Yokoyama [14, Lemma 3.1]). Thus we assume $\varphi=\psi \equiv 0$. Then the case $\mu \geq 1$ is treated in Kubota-Yokoyama [18, Lemma 3.2 , and the other case can be treated similarly. Here we give the outline of the proof.

The solution $u$ with $\varphi=\psi \equiv 0$ can be written as

$$
u(t, x)=\frac{1}{4 \pi r} \int_{0}^{t}\left(\int_{|r-(t-\tau)|}^{r+t-\tau}\left(\int_{0}^{2 \pi} F(\tau, \lambda \Theta(\lambda, \theta ; t-\tau, x)) d \theta\right) \lambda d \lambda\right) d \tau,
$$

where we put $r=|x|$, and $\Theta$ is a certain $S^{2}$-valued function with

$$
|\lambda \Theta(\lambda, \theta ; s, x)-x|=s
$$

(see John [10]). Therefore (3.19) with $\varphi=\psi \equiv 0$ is obtained if we can show

$$
\begin{aligned}
J_{\mu, \kappa}(t, r) & :=\frac{1}{r} \int_{0}^{t}\left(\int_{|r-(t-\tau)|}^{r+t-\tau}(1+\tau+\lambda)^{-\mu}(1+|\tau-\lambda|)^{-\kappa} d \lambda\right) d \tau \\
& \leq C\langle t+r\rangle^{-1} \mathcal{W}_{\mu-1}(t, r)^{-1}
\end{aligned}
$$

with some positive constant $C$. We put $p=\tau+\lambda$ and $q=\lambda-\tau$. Then we have

$$
\begin{aligned}
J_{\mu, \kappa}(t, r) & =\frac{1}{2 r} \int_{|t-r|}^{t+r}(1+p)^{-\mu}\left(\int_{r-t}^{p}(1+|q|)^{-\kappa} d q\right) d p \\
& \leq \frac{1}{(\kappa-1) r} \int_{|t-r|}^{t+r}(1+p)^{-\mu} d p
\end{aligned}
$$

where we have used the assumption $\kappa>1$. Now, if $r \geq(1+t) / 2$, then a direct calculation of the last integral in (3.21) leads to (3.20), since we have $r^{-1} \leq 3(1+$ $t+r)^{-1}$ for this case. If $r<(1+t) / 2$, then we have $(1+|t-r|)^{-1} \leq(1+t-r)^{-1} \leq$ $3(1+t+r)^{-1}$, and we get

$$
\frac{1}{r} \int_{|t-r|}^{t+r}(1+p)^{-\mu} d p \leq(1+|t-r|)^{-\mu} \frac{1}{r} \int_{|t-r|}^{t+r} d p \leq C(1+t+r)^{-\mu}
$$

which implies the desired result. 


\subsection{The Friedlander radiation field and the translation representation.}

It is known that if $(\varphi, \psi) \in C_{0}^{\infty}\left(\mathbb{R}^{3}\right) \times C_{0}^{\infty}\left(\mathbb{R}^{3}\right)$, then the radiation field $\mathcal{F}_{0}[\varphi, \psi]$, given by (1.13), belongs to $C_{0}^{\infty}\left(\mathbb{R} \times S^{2}\right)$. In fact, if $\operatorname{supp} \varphi \cup \operatorname{supp} \psi \subset B_{R}$ for some $R>0$, then (1.13) and (1.14) imply

$$
\mathcal{F}_{0}[\varphi, \psi](\sigma, \omega)=0, \quad|\sigma| \geq R, \omega \in S^{2} .
$$

We define the mapping $\mathcal{T}: C_{0}^{\infty}\left(\mathbb{R}^{3}\right) \times C_{0}^{\infty}\left(\mathbb{R}^{3}\right) \rightarrow C_{0}^{\infty}\left(\mathbb{R} \times S^{2}\right)$ by

$$
\mathcal{T}[\varphi, \psi](\sigma, \omega):=\partial_{\sigma} \mathcal{F}_{0}[\varphi, \psi](\sigma, \omega), \quad(\sigma, \omega) \in \mathbb{R} \times S^{2}
$$

for $(\varphi, \psi) \in C_{0}^{\infty}\left(\mathbb{R}^{3}\right) \times C_{0}^{\infty}\left(\mathbb{R}^{3}\right) . \mathcal{T}[\varphi, \psi]$ is called the translation representation of $(\varphi, \psi)$, because we have

$$
\mathcal{T}\left[\mathcal{U}_{0}[\varphi, \psi](t, \cdot), \partial_{t} \mathcal{U}_{0}[\varphi, \psi](t, \cdot)\right](\sigma, \omega)=\mathcal{T}[\varphi, \psi](\sigma-t, \omega)
$$

where $\mathcal{U}_{0}[\varphi, \psi]$ is defined in Section 2.1 (namely it is the solution to (1.10)-(1.11) ).

Let $H_{0}$ be the completion of $C_{0}^{\infty}\left(\mathbb{R}^{3}\right) \times C_{0}^{\infty}\left(\mathbb{R}^{3}\right)$ with respect to the norm

$$
\|(\varphi, \psi)\|_{H_{0}}:=\left(\frac{1}{2} \int_{\mathbb{R}^{3}}\left(\left|\nabla_{x} \varphi(x)\right|^{2}+|\psi(x)|^{2}\right) d x\right)^{1 / 2} .
$$

Then it is known that $\mathcal{T}$ is uniquely extended to an isometric isomorphism from $H_{0}$ onto $L^{2}\left(\mathbb{R} \times S^{2}\right)$. We refer the readers to Lax-Phillips [19, Chapter IV] for the facts mentioned above. Note that $(\varphi, \psi) \in H_{0}$ if and only if $\varphi \in \dot{H}^{1}\left(\mathbb{R}^{3}\right)$ and $\psi \in L^{2}\left(\mathbb{R}^{3}\right)$.

Lemma 3.10. Let $(\varphi, \psi) \in H_{0}$. Then we have

$$
\lim _{t \rightarrow \infty}\left(\frac{1}{2} \sum_{a=0}^{3}\left\|\partial_{a} u_{0}(t, \cdot)-T_{*}^{a}(t, \cdot)\right\|_{L^{2}\left(\mathbb{R}^{3}\right)}^{2}\right)^{1 / 2}=0,
$$

where

$$
\begin{aligned}
u_{0}(t, x) & =\mathcal{U}_{0}[\varphi, \psi](t, x), \\
T_{*}^{a}(t, x) & =\left.\left(\omega_{a} r^{-1} \mathcal{T}[\varphi, \psi](r-t, \omega)\right)\right|_{r=|x|, \omega=\left(\omega_{1}, \omega_{2}, \omega_{3}\right)=x /|x|}, \quad 0 \leq a \leq 3 .
\end{aligned}
$$

Proof. Let $\varepsilon>0$. Then there exists $(\widetilde{\varphi}, \widetilde{\psi}) \in C_{0}^{\infty}\left(\mathbb{R}^{3}\right) \times C_{0}^{\infty}\left(\mathbb{R}^{3}\right)$ such that

$$
\|(\varphi, \psi)-(\widetilde{\varphi}, \widetilde{\psi})\|_{H_{0}}<\varepsilon \text {. }
$$

Let $\widetilde{u}_{0}$ and $\widetilde{T}_{*}^{a}$ be defined similarly to $u_{0}$ and $T_{*}^{a}$ by replacing $(\varphi, \psi)$ with $(\widetilde{\varphi}, \widetilde{\psi})$ in their definitions. Suppose that $\operatorname{supp} \widetilde{\varphi} \cup \operatorname{supp} \widetilde{\psi} \subset B_{R}$ with $R>0$. Then the Huygens principle implies $\widetilde{u}_{0}(t, x)=0$ for $|r-t| \geq R$ with $r=|x|$. We also have $\widetilde{T}_{*}^{a}(t, x)=0$ for $|r-t| \geq R($ see $(\underline{3.22}))$. Hence, for $t \geq \max \{2 R, 2\}$, (1.15) leads to

$$
\begin{aligned}
& \left\|\partial_{a} \widetilde{u}_{0}(t, \cdot)-\widetilde{T}_{*}^{a}(t, \cdot)\right\|_{L^{2}\left(\mathbb{R}^{3}\right)}^{2} \\
& \quad=\int_{\omega \in S^{2}}\left(\int_{t-R}^{t+R}\left|r \partial_{a} \widetilde{u}_{0}(t, r \omega)-\omega_{a} \partial_{\sigma} \mathcal{F}_{0}[\widetilde{\varphi}, \widetilde{\psi}](r-t, \omega)\right|^{2} d r\right) d S_{\omega} \\
& \quad \leq C R(1+t)^{-2},
\end{aligned}
$$


since we have $t-R \geq t / 2 \geq 1$. Here and hereafter $d S_{\omega}$ denotes the area element on $S^{2}$. From the energy identity and (3.23) we get

$$
\left\|u_{0}(t, \cdot)-\widetilde{u}_{0}(t, \cdot)\right\|_{E}=\|(\varphi, \psi)-(\widetilde{\varphi}, \widetilde{\psi})\|_{H_{0}}<\varepsilon .
$$

Since $\mathcal{T}$ is an isometry from $H_{0}$ to $L^{2}\left(\mathbb{R} \times S^{2}\right)$, by (3.23) we obtain

$$
\begin{aligned}
\frac{1}{2} \sum_{a=0}^{3}\left\|T_{*}^{a}(t, \cdot)-\widetilde{T}_{*}^{a}(t, \cdot)\right\|_{L^{2}\left(\mathbb{R}^{3}\right)}^{2} & =\int_{\omega \in S^{2}}\left(\int_{0}^{\infty}|\mathcal{T}[\varphi-\widetilde{\varphi}, \psi-\widetilde{\psi}](r-t, \omega)|^{2} d r\right) d S_{\omega} \\
& \leq\|\mathcal{T}[\varphi-\widetilde{\varphi}, \psi-\widetilde{\psi}]\|_{L^{2}\left(\mathbb{R} \times S^{2}\right)}^{2}<\varepsilon^{2} .
\end{aligned}
$$

From (3.24), (3.25), and (3.26) we get

$$
\limsup _{t \rightarrow \infty}\left(\frac{1}{2} \sum_{a=0}^{3}\left\|\partial_{a} u_{0}(t, \cdot)-T_{*}^{a}(t, \cdot)\right\|_{L^{2}\left(\mathbb{R}^{3}\right)}^{2}\right)^{1 / 2} \leq 2 \varepsilon .
$$

Since $\varepsilon>0$ can be chosen arbitrarily, we obtain the desired result.

\section{Proof of Proposition 2.3}

In this section, we are going to prove Proposition 2.3 .

Proof of (1). Let the assumptions in (1) be fulfilled. Then, as in (1.6), $F_{j}^{0}$ can be written in terms of the null forms. Hence it is clear by Lemma 3.3 that we have

$$
F_{j}^{0}(\partial u) \stackrel{\mathcal{X}_{N}}{\sim} 0, \quad 1 \leq j \leq N
$$

which implies the desired result immediately.

Proof of (2). Suppose that $F$ depends only on $\partial u$, i.e., $F=F(\partial u)$, and that Condition 1.1 is satisfied. As before, we write $F^{\text {red }}=F^{\text {red }}(\omega, Y)$ for the reduced nonlinearity. Let $u=\left(u_{j}\right)_{1 \leq j \leq N}$ be a (local) solution to (1.1) with the initial data (2.8). Set

$$
v=\left(v_{j}\right)_{1 \leq j \leq 5 N}^{\mathrm{T}}=\left(u^{\mathrm{T}}, \partial_{0} u^{\mathrm{T}}, \partial_{1} u^{\mathrm{T}}, \partial_{2} u^{\mathrm{T}}, \partial_{3} u^{\mathrm{T}}\right)^{\mathrm{T}}, w=\left(w_{l}\right)_{1 \leq l \leq N_{0}}^{\mathrm{T}}
$$

with

$$
w_{l}=\sum_{a=0}^{3} \sum_{k=1}^{N} h_{l, k a}\left(\partial_{a} u_{k}\right), \quad 1 \leq l \leq N_{0},
$$

where the constants $h_{l, k a}$ are from (1.18). We put $u^{*}=\left(u_{j}^{*}\right)_{1 \leq j \leq N^{*}}^{\mathrm{T}}=\left(v^{\mathrm{T}}, w^{\mathrm{T}}\right)^{\mathrm{T}}$ with $N^{*}=5 N+N_{0}$. Given $(f, g) \in \mathcal{X}_{N}$, from (1.1) and (2.8) we can determine the initial profile $\left(\varepsilon^{-1} u^{*}(0, x), \varepsilon^{-1} \partial_{t} u^{*}(0, x)\right)$ for $\varepsilon \in(0,1]$, and we denote this initial profile by $\left(f^{*}(x ; \varepsilon), g^{*}(x ; \varepsilon)\right)=\left(f^{*}[f, g](x ; \varepsilon), g^{*}[f, g](x ; \varepsilon)\right)$. Since we can see that $\left(f^{*}, g^{*}\right)$ converges to a $C_{0}^{\infty} \times C_{0}^{\infty}$-function as $\varepsilon \rightarrow+0$, we define $\left(f^{*}(x ; 0), g^{*}(x ; 0)\right)=$ $\lim _{\varepsilon \rightarrow+0}\left(f^{*}(x ; \varepsilon), g^{*}(x ; \varepsilon)\right)$. Then it is easy to show that $\left(f^{*}, g^{*}\right) \in \mathcal{X}_{N^{*}}$. Note that 
the first $N$ components of $f^{*}$ (resp. $g^{*}$ ) are nothing but $f$ (resp. $g$ ) by definition. Now we define a subset $D$ of $\mathcal{X}_{N^{*}}$ by

$$
D=\left\{\left(f^{*}[f, g], g^{*}[f, g]\right) ;(f, g) \in \mathcal{X}_{N}\right\} .
$$

Since $F_{j}$ is a homogeneous polynomial of degree 2 , we can write

$$
F_{j}(\partial u)=\sum_{k, l=1}^{N} \sum_{b, c=0}^{3} q_{j}^{k b, l c}\left(\partial_{b} u_{k}\right)\left(\partial_{c} u_{l}\right)
$$

with some constants $q_{j}^{k b, l c}$. Recall that $v_{j}=u_{j}$ and $v_{j+(a+1) N}=\partial_{a} u_{j}$ for $1 \leq j \leq N$ and $0 \leq a \leq 3$. We put

$$
\begin{aligned}
F_{j}^{*}\left(\partial u^{*}\right) & :=\sum_{k, l=1}^{N} \sum_{b, c=0}^{3} q_{j}^{k b, l c}\left(\partial_{b} v_{k}\right)\left(\partial_{c} v_{l}\right) \\
F_{j+(a+1) N}^{*}\left(\partial u^{*}\right) & :=\sum_{k, l=1}^{N} \sum_{b, c=0}^{3} q_{j}^{k b, l c}\left(\left(\partial_{b} v_{k}\right)\left(\partial_{c} v_{l+(a+1) N}\right)+\left(\partial_{b} v_{k+(a+1) N}\right)\left(\partial_{c} v_{l}\right)\right)
\end{aligned}
$$

for $1 \leq j \leq N$ and $0 \leq a \leq 3$, so that we have

$$
F_{j}^{*}\left(\partial u^{*}\right)=F_{j}(\partial u) \text { and } F_{j+(a+1) N}^{*}\left(\partial u^{*}\right)=\partial_{a}\left(F_{j}(\partial u)\right) .
$$

Then we find that $u^{*}$ satisfies the extended system

$$
\begin{cases}\square v_{j}=F_{j}^{*}\left(\partial u^{*}\right), & 1 \leq j \leq N, \\ \square v_{j+(a+1) N}=F_{j+(a+1) N}^{*}\left(\partial u^{*}\right), & 1 \leq j \leq N, 0 \leq a \leq 3, \\ \square w_{l}=\sum_{a=0}^{3} \sum_{k=1}^{N} h_{l, k a} F_{k+(a+1) N}^{*}\left(\partial u^{*}\right), & 1 \leq l \leq N_{0}\end{cases}
$$

with initial data

$$
u^{*}(0, x)=\varepsilon f^{*}[f, g](x ; \varepsilon),\left(\partial_{t} u^{*}\right)(0, x)=\varepsilon g^{*}[f, g](x ; \varepsilon), \quad x \in \mathbb{R}^{3} .
$$

Conversely, let $u^{*}=\left(u_{j}^{*}\right)_{1 \leq j \leq N^{*}}^{\mathrm{T}}=\left(v^{\mathrm{T}}, w^{\mathrm{T}}\right)^{\mathrm{T}}$ be the classical solution to (4.6)(4.7). If we put

$$
u=\left(u_{j}\right)_{1 \leq j \leq N}^{\mathrm{T}}:=\left(u_{j}^{*}\right)_{1 \leq j \leq N}^{\mathrm{T}}\left(=\left(v_{j}\right)_{1 \leq j \leq N}^{\mathrm{T}}\right),
$$

then $u$ is apparently the solution to the original problem (1.1)-(2.8). Moreover, from the uniqueness of the solution we find that (4.2), (4.3), and (4.5) are valid for all $t \geq 0$. To sum up, we have proved that solving (1.1)-(2.8) with $(f, g) \in \mathcal{X}_{N}$ is equivalent to solving (4.6)-(4.7) with $\left(f^{*}, g^{*}\right) \in D\left(\subset \mathcal{X}_{N^{*}}\right)$.

Now we are going to prove that Condition 2.2 is satisfied for (4.6), if it is viewed as a system of $u^{*}$. It is trivial to check the condition (2.11), and we concentrate on the condition (2.13). Let $u^{*}=\left(v^{\mathrm{T}}, w^{\mathrm{T}}\right)^{\mathrm{T}}$ be the solution to (4.6)-(4.7), and let $u$ be given by (4.8). As before, we write $r=|x|$, and $\omega=|x|^{-1} x=\left(\omega_{1}, \omega_{2}, \omega_{3}\right) \in S^{2}$. We always assume $r \geq t / 2 \geq 1$ in this subsection from now on. Hence we have

$$
r^{-1} \leq 4(1+t+r)^{-1} \text {. }
$$

Note that $\Phi \stackrel{D}{\sim} \Psi$ in this proof means (2.9) with $u$ being replaced by $u^{*}$. 
For $N \times 4$-matrix valued functions $\varphi=\left(\varphi_{j, a}\right)$ and $\psi=\left(\psi_{j, a}\right)$ with $1 \leq j \leq N$ and $0 \leq a \leq 3$, we define

$$
F_{j}^{\dagger}(\varphi, \psi):=\sum_{k, l=1}^{N} \sum_{b, c=0}^{3} q_{j}^{k b, l c} \varphi_{k, b} \psi_{l, c}, \quad 1 \leq j \leq N
$$

with the coefficients $q_{j}^{k b, l c}$ from (4.4), so that we have

$$
F_{j}^{*}\left(\partial u^{*}\right)=F_{j}(\partial u)=F_{j}^{\dagger}(\partial u, \partial u), \quad 1 \leq j \leq N
$$

by regarding $\partial u=\left(\partial_{a} u_{j}\right)_{1 \leq j \leq N, 0 \leq a \leq 3}$ as an $N \times 4$-matrix valued function. We put $Z_{0}=0\left(=\omega_{0} \partial_{t}+\partial_{0}\right)$. Then from (3.13) we get

$$
\partial_{a}=Z_{a}-\omega_{a} \partial_{t}, \quad 0 \leq a \leq 3
$$

We write $Z^{\star} u=\left(Z_{a} u_{j}\right)_{1 \leq j \leq N, 0 \leq a \leq 3}$, and $\omega^{\star} \partial_{t} u=\left(\omega_{a} \partial_{t} u_{j}\right)_{1 \leq j \leq N, 0 \leq a \leq 3}$. Regarding them as $N \times 4$ matrix-valued functions, we obtain

$$
F_{j}(\partial u)=F_{j}^{\mathrm{red}}\left(\omega, \partial_{t} u\right)-F_{j}^{\dagger}\left(Z^{\star} u, \omega^{\star} \partial_{t} u\right)-F_{j}^{\dagger}\left(\omega^{\star} \partial_{t} u, Z^{\star} u\right)+F_{j}^{\dagger}\left(Z^{\star} u, Z^{\star} u\right),
$$

where we have used $F_{j}^{\dagger}\left(-\omega^{\star} \partial_{t} u,-\omega^{\star} \partial_{t} u\right)=F_{j}^{\text {red }}\left(\omega, \partial_{t} u\right)$. Let $s$ be a nonnegative integer. Since we have $\sup _{|x| \geq t / 2 \geq 1}|\omega|_{s}<\infty$, it follows from (3.15) in Lemma 3.7 that

$$
\begin{aligned}
\left|F_{j}^{\dagger}\left(Z^{\star} u, \omega^{\star} \partial_{t} u\right)\right|_{s} & \leq C\left(|\partial u|_{[s / 2]}\left|Z^{\star} u\right|_{s}+\left|Z^{\star} u\right|_{[s / 2]}|\partial u|_{s}\right) \\
& \leq C\langle t+r\rangle^{-1}\left(|\partial u|_{[s / 2]}|u|_{s+1}+|u|_{[s / 2]+1}|\partial u|_{s}\right)
\end{aligned}
$$

which implies

$$
F_{j}^{\dagger}\left(Z^{\star} u, \omega^{\star} \partial_{t} u\right) \stackrel{D}{\sim} 0, \quad 1 \leq j \leq N
$$

In a similar manner we can show that

$$
F_{j}^{\dagger}\left(\omega^{\star} \partial_{t} u, Z^{\star} u\right) \stackrel{D}{\sim} F_{j}^{\dagger}\left(Z^{\star} u, Z^{\star} u\right) \stackrel{D}{\sim} 0
$$

and we get

$$
F_{j}^{*}\left(\partial u^{*}\right)=F_{j}(\partial u) \stackrel{D}{\sim} F_{j}^{\mathrm{red}}\left(\omega, \partial_{t} u\right), \quad 1 \leq j \leq N .
$$

Similarly to (4.11), using (3.16) in Lemma 3.7 as well as (3.15), and recalling (4.2), we find from (4.10) that

$$
\begin{aligned}
& \left|\partial_{a}\left(F_{j}(\partial u)\right)-\partial_{a}\left(F_{j}^{\mathrm{red}}\left(\omega, \partial_{t} u\right)\right)\right|_{s} \\
& \quad \leq C\langle t+r\rangle^{-1}\left(\left|\partial u^{*}\right|_{[s / 2]}\left|u^{*}\right|_{s+1}+\left|u^{*}\right|_{[s / 2]+1}\left|\partial u^{*}\right|_{s}\right) .
\end{aligned}
$$

In other words, we obtain

$$
\partial_{a}\left(F_{j}(\partial u)\right) \stackrel{D}{\sim} \partial_{a}\left(F_{j}^{\mathrm{red}}\left(\omega, \partial_{t} u\right)\right), \quad 1 \leq j \leq N, 0 \leq a \leq 3 .
$$

From (1.18) and (4.3), we get

$$
h_{l}\left(\omega, \partial_{t} u\right)+w_{l}=\sum_{a=0}^{3} \sum_{k=1}^{N} h_{l, k a} Z_{a} u_{k}, \quad 1 \leq l \leq N_{0}
$$


Now we define $G_{j}\left(\omega, u^{*}, \partial u^{*}\right)$ for $1 \leq j \leq 5 N$ by

$$
G_{j}:=-\sum_{l=1}^{N_{0}} \sum_{k=1}^{N} g_{j l, k}(\omega) w_{l}\left(\partial_{t} v_{k}\right)\left(=-\sum_{l=1}^{N_{0}} g_{j l}\left(\omega, \partial_{t} u\right) w_{l}\right)
$$

for $1 \leq j \leq N$, and

$$
G_{j+(a+1) N}:=-\sum_{l=1}^{N_{0}} \sum_{k=1}^{N} g_{j l, k}(\omega)\left\{\left(\partial_{a} w_{l}\right)\left(\partial_{t} v_{k}\right)+w_{l}\left(\partial_{t} v_{k+(a+1) N}\right)\right\}
$$

for $1 \leq j \leq N$ and $0 \leq a \leq 3$, where $g_{j l, k}(\omega)$ and $g_{j l}(\omega, Y)$ are from (1.17). Then, by (1.20) in Condition 1.1 and (4.14) we obtain

$$
F_{j}^{\mathrm{red}}\left(\omega, \partial_{t} u\right)-G_{j}\left(\omega, u^{*}, \partial u^{*}\right)=\sum_{a=0}^{3} \sum_{l=1}^{N_{0}} \sum_{k=1}^{N} h_{l, k a} g_{j l}\left(\omega, \partial_{t} u\right)\left(Z_{a} u_{k}\right)
$$

for $1 \leq j \leq N$. Going a similar way to (4.11), and using (4.12), we obtain

$$
F_{j}^{*}\left(\partial u^{*}\right) \stackrel{D}{\sim} F_{j}^{\mathrm{red}}\left(\omega, \partial_{t} u\right) \stackrel{D}{\sim} G_{j}\left(\omega, u^{*}, \partial u^{*}\right), \quad 1 \leq j \leq N
$$

Since we have

$$
\sup _{|x| \geq t / 2 \geq 1} r\left|\partial_{a}\left(g_{j l, k}(\omega)\right)\right|_{s} \leq C
$$

and

$$
G_{j+(a+1) N}\left(\omega, u^{*}, \partial u^{*}\right)=\partial_{a}\left(G_{j}\left(\omega, u^{*}, \partial u^{*}\right)\right)+\sum_{l=1}^{N_{0}} \sum_{k=1}^{N}\left(\partial_{a}\left(g_{j l, k}(\omega)\right)\right) w_{l}\left(\partial_{t} u_{k}\right)
$$

for $1 \leq j \leq N$ and $0 \leq a \leq 3$ (cf. (4.2)), it follows from Lemma 3.7 and (4.17) that

$$
\partial_{a}\left(F_{j}^{\mathrm{red}}\left(\omega, \partial_{t} u\right)\right) \stackrel{D}{\sim} G_{j+(a+1) N}\left(\omega, u^{*}, \partial u^{*}\right), \quad 1 \leq j \leq N, 0 \leq a \leq 3 .
$$

Combining this with (4.13), and remembering (4.5), we obtain

$$
F_{j+(a+1) N}^{*}\left(\partial u^{*}\right)=\partial_{a}\left(F_{j}(\partial u)\right) \stackrel{D}{\sim} G_{j+(a+1) N}\left(\omega, u^{*}, \partial u^{*}\right)
$$

for $1 \leq j \leq N$ and $0 \leq a \leq 3$.

Now what is left to prove is

$$
\sum_{a=0}^{3} \sum_{k=1}^{N} h_{l, k a} F_{k+(a+1) N}^{*}\left(\partial u^{*}\right) \stackrel{D}{\sim} 0, \quad 1 \leq l \leq N_{0}
$$

We define $\widetilde{q}_{j}^{k l}(\omega)=\sum_{b, c=0}^{3}\left(q_{j}^{k b, l c}+q_{j}^{l b, k c}\right) \omega_{b} \omega_{c}$ for $1 \leq k<l \leq N$, and $\widetilde{q}_{j}^{k k}(\omega)=$ $\sum_{b, c=0}^{3} q_{j}^{k b, k c} \omega_{b} \omega_{c}$ for $1 \leq k \leq N$ so that we can write

$$
F_{j}^{\mathrm{red}}(\omega, Y)=\sum_{1 \leq k \leq l \leq N} \widetilde{q}_{j}^{k l}(\omega) Y_{k} Y_{l}
$$


Let $\beta=\left(\beta_{j}(\omega)\right)_{1 \leq j \leq N}^{\mathrm{T}}$ and $M=M(\omega, Y)$ be from the condition (1.19). Since $M$ is quadratic in $Y$, we can write it as

$$
M(\omega, Y)=\sum_{1 \leq k \leq l \leq N} m_{k l}(\omega) Y_{k} Y_{l}
$$

with some coefficients $m_{k l}(\omega)$. Then from (1.19) we find

$$
\widetilde{q}_{j}^{k l}(\omega)=\beta_{j}(\omega) m_{k l}(\omega), \quad 1 \leq j \leq N, 1 \leq k \leq l \leq N .
$$

Observing that we have $\left|\partial_{a}\left(\widetilde{q}_{j}^{k l}(\omega)\right)\right|_{s} \leq C r^{-1}$, and that Lemma 3.7 yields

$$
\left|Z_{a} \partial_{t} u_{k}\right|_{s}=\left|Z_{a} v_{k+N}\right|_{s} \leq C\langle t+r\rangle^{-1}\left|u^{*}\right|_{s+1},
$$

we obtain from (4.9) and (4.21) that

$$
\begin{aligned}
& \partial_{a}\left(F_{j}^{\mathrm{red}}\left(\omega, \partial_{t} u\right)\right)= \sum_{1 \leq k \leq l \leq N}\left(\partial_{a}\left(\widetilde{q}_{j}^{k l}(\omega)\right)\right)\left(\partial_{t} u_{k}\right)\left(\partial_{t} u_{l}\right) \\
&+\sum_{1 \leq k \leq l \leq N} \widetilde{q}_{j}^{k l}(\omega)\left(\left(\partial_{a} \partial_{t} u_{k}\right)\left(\partial_{t} u_{l}\right)+\left(\partial_{t} u_{k}\right)\left(\partial_{a} \partial_{t} u_{l}\right)\right) \\
& \stackrel{D}{\sim}-\omega_{a} \sum_{1 \leq k \leq l \leq N} \widetilde{q}_{j}^{k l}(\omega)\left(\left(\partial_{t}^{2} u_{k}\right)\left(\partial_{t} u_{l}\right)+\left(\partial_{t} u_{k}\right)\left(\partial_{t}^{2} u_{l}\right)\right) \\
&=-\omega_{a} \beta_{j}(\omega) \widetilde{M}\left(\omega, \partial_{t} u, \partial_{t}^{2} u\right),
\end{aligned}
$$

where we have set

$$
\widetilde{M}\left(\omega, \partial_{t} u, \partial_{t}^{2} u\right)=\sum_{1 \leq k \leq l \leq N} m_{k l}(\omega)\left(\left(\partial_{t}^{2} u_{k}\right)\left(\partial_{t} u_{l}\right)+\left(\partial_{t} u_{k}\right)\left(\partial_{t}^{2} u_{l}\right)\right) .
$$

Hence from (4.5), (4.13), and (4.22), we get

$$
\begin{aligned}
\sum_{a=0}^{3} \sum_{k=1}^{N} h_{l, k a} F_{k+(a+1) N}^{*}\left(\partial u^{*}\right) & \stackrel{D}{\sim} \sum_{a=0}^{3} \sum_{k=1}^{N} h_{l, k a} \partial_{a}\left(F_{k}^{\mathrm{red}}\left(\omega, \partial_{t} u\right)\right) \\
& \stackrel{D}{\sim}-\sum_{a=0}^{3} \sum_{k=1}^{N} h_{l, k a} \omega_{a} \beta_{k}(\omega) \widetilde{M}\left(\omega, \partial_{t} u, \partial_{t}^{2} u\right) \\
& =-h_{l}(\omega, \beta(\omega)) \widetilde{M}\left(\omega, \partial_{t} u, \partial_{t}^{2} u\right)=0, \quad 1 \leq l \leq N_{0},
\end{aligned}
$$

where the last identity comes from (1.21). This completes the proof.

\section{Proof of Theorem 2.5}

Suppose that the assumptions in Theorem 2.5 are fulfilled. Let $(f, g) \in D(\subset$ $\left.\mathcal{X}_{N}\right)$, and let $u=\left(v^{\mathrm{T}}, w^{\mathrm{T}}\right)^{\mathrm{T}}$ be a (local) solution to (1.1)-(2.8) in $\left[0, T_{0}\right) \times \mathbb{R}^{3}$ with some $T_{0}>0$. Since $u$ depends on the parameter $\varepsilon$ from (2.8), we sometimes write $u=u(t, x ; \varepsilon)$ if we want to indicate the dependence of $u$ on $\varepsilon$ explicitly, but we sometimes omit $\varepsilon$ and only write $u=u(t, x)$ for simplicity of expression. Similar 
notation will be used for functions depending on the parameter $\varepsilon$ in what follows. Since $(f, g) \in \mathcal{X}_{N}$, there is a positive constant $R$ such that

$$
\operatorname{supp} f(\cdot ; \varepsilon) \cup \operatorname{supp} g(\cdot ; \varepsilon) \subset B_{R}, \quad \varepsilon \in(0,1],
$$

where $B_{R}$ is defined by (3.12). By the finite propagation property, (5.1) implies

$$
\operatorname{supp} u(t, \cdot ; \varepsilon) \subset B_{t+R}, \quad t \in\left[0, T_{0}\right) .
$$

We set

$$
u_{j}^{0}(t, x ; \varepsilon):=\mathcal{U}_{0}\left[f_{j}(\cdot ; \varepsilon), g_{j}(\cdot ; \varepsilon)\right](t, x), \quad \widetilde{u}_{j}(t, x ; \varepsilon):=u_{j}(t, x ; \varepsilon)-\varepsilon u_{j}^{0}(t, x ; \varepsilon)
$$

for $1 \leq j \leq N$. In other words, $u_{j}^{0}(t, x ; \varepsilon)$ is the solution to the free wave equation $\square u_{j}^{0}=0$ with the initial condition

$$
u_{j}^{0}(0, x ; \varepsilon)=f_{j}(x ; \varepsilon),\left(\partial_{t} u_{j}^{0}\right)(0, x ; \varepsilon)=g_{j}(x ; \varepsilon), \quad 1 \leq j \leq N .
$$

We put $u^{0}=\left(u_{j}^{0}\right)_{1 \leq j \leq N}^{\mathrm{T}}$ and $\widetilde{u}=\left(\widetilde{u}_{j}\right)_{1 \leq j \leq N}^{\mathrm{T}}$. We also define

$$
\begin{aligned}
\left(\left(v^{0}\right)^{\mathrm{T}},\left(w^{0}\right)^{\mathrm{T}}\right) & =\left(v_{1}^{0}, \ldots, v_{N^{\prime}}^{0}, w_{1}^{0}, \ldots, w_{N^{\prime \prime}}^{0}\right):=\left(u_{1}^{0}, \ldots, u_{N^{\prime}}^{0}, u_{N^{\prime}+1}^{0}, \ldots, u_{N}^{0}\right), \\
\left(\widetilde{v}^{\mathrm{T}}, \widetilde{w}^{\mathrm{T}}\right) & =\left(\widetilde{v}_{1}, \ldots, \widetilde{v}_{N^{\prime}}, \widetilde{w}_{1}, \ldots, \widetilde{w}_{N^{\prime \prime}}\right):=\left(\widetilde{u}_{1}, \ldots, \widetilde{u}_{N^{\prime}}, \widetilde{u}_{N^{\prime}+1}, \ldots, \widetilde{u}_{N}\right) .
\end{aligned}
$$

Recall the definition of $|\cdot|_{s},\|\cdot\|_{s}$, and $|\cdot|_{Z, s}$ given in (2.6) and (3.14). Let $m, \lambda$, and $\rho$ be the fixed constants from the assumptions of Theorem 2.5. For $0<T \leq T_{0}$, we define

$$
\begin{aligned}
e_{\varepsilon}[\widetilde{u}](T):= & \sup _{(t, x) \in[0, T) \times \mathbb{R}^{3}}\langle t+|x|\rangle^{-\lambda+1}|\widetilde{v}(t, x ; \varepsilon)|_{m+1} \\
& +\sup _{(t, x) \in[0, T) \times \mathbb{R}^{3}}\langle t+|x|\rangle\langle t-|x|\rangle^{\rho}|\widetilde{w}(t, x ; \varepsilon)|_{m+2} \\
& +\sup _{t \in[0, T)}\left((1+t)^{-\lambda}\|\partial \widetilde{v}(t, \cdot ; \varepsilon)\|_{2 m}+\|\partial \widetilde{w}(t, \cdot ; \varepsilon)\|_{2 m}\right) \\
& +\left(\int_{0}^{T} \int_{\mathbb{R}^{3}} \frac{|\widetilde{u}(t, x ; \varepsilon)|_{Z, 2 m}^{2}}{\langle t\rangle^{4 \lambda}\langle t-|x|\rangle^{2}} d x d t\right)^{1 / 2} .
\end{aligned}
$$

For a smooth function $\varphi=\varphi(x)$ and a nonnegative integer $s$, we define

$$
|\varphi(x)|_{s, *}:=\sum_{|\alpha| \leq s}\langle x\rangle^{|\alpha|}\left|\partial_{x}^{\alpha} \varphi(x)\right|, \quad\|\varphi\|_{s, *}:=\left(\sum_{|\alpha| \leq s}\left\|\langle\cdot\rangle^{|\alpha|} \partial_{x}^{\alpha} \varphi\right\|_{L^{2}}^{2}\right)^{1 / 2}
$$

and we put

$$
\begin{aligned}
M_{0}:= & \sup _{\varepsilon \in[0,1]}\left(\|f(\cdot ; \varepsilon)\|_{2 m+1, *}+\|g(\cdot ; \varepsilon)\|_{2 m, *}\right) \\
& +\sup _{\varepsilon \in[0,1], x \in \mathbb{R}^{3}}\langle x\rangle^{3}\left(|f(x ; \varepsilon)|_{2 m-1, *}+|g(x, \varepsilon)|_{2 m-2, *}\right) .
\end{aligned}
$$


Lemmas 3.8 and 3.9 yield

$$
\begin{aligned}
& \sup _{(t, x) \in \mathbb{R}_{+} \times \mathbb{R}^{3}}\langle t+|x|\rangle\langle t-|x|\rangle\left|u^{0}(t, x ; \varepsilon)\right|_{2 m-2} \\
& +\sup _{t \in \mathbb{R}_{+}}\left\|\partial u^{0}(t, \cdot ; \varepsilon)\right\|_{2 m}+\left(\int_{0}^{\infty} \int_{\mathbb{R}^{3}} \frac{\left|u^{0}(t, x ; \varepsilon)\right|_{Z, 2 m}^{2}}{\langle t-|x|\rangle^{2}} d x d t\right)^{1 / 2} \leq C_{0}
\end{aligned}
$$

for $\varepsilon \in(0,1]$, where $C_{0}$ is a positive constant depending only on $M_{0}$.

We are going to prove the following:

Proposition 5.1. In the situation above, there exists a positive constant $A_{0}=$ $A_{0}\left(M_{0}, R\right)$, which is independent of $T_{0}$, such that

$$
e_{\varepsilon}[\widetilde{u}](T) \leq A \varepsilon^{2}
$$

implies

$$
e_{\varepsilon}[\widetilde{u}](T) \leq \frac{A}{2} \varepsilon^{2},
$$

provided that $A \geq A_{0}, 0<\varepsilon \leq \min \{1,1 / A\}$, and $0<T<T_{0}$.

Once we have the proposition above, we can easily obtain Theorem 2.5 in the following way.

Proof of Theorem 2.5. If $A\left(\geq A_{0}\right)$ is sufficiently large, then (5.5) is true for some small $T>0$. Let $T_{*}$ be the supremum of all $T \in\left(0, T_{0}\right)$ such that (5.5) is valid. Note that we have $T_{*}>0$. Let $\varepsilon \in\left(0, \varepsilon_{0}\right]$ with $\varepsilon_{0}:=\min \{1,1 / A\}$. Suppose that we have $T_{*}<T_{0}$; then, since we have $e_{\varepsilon}[\widetilde{u}]\left(T_{*}\right) \leq A \varepsilon^{2}$, Proposition 5.1 implies that $e_{\varepsilon}[\widetilde{u}]\left(T_{*}\right) \leq A \varepsilon^{2} / 2$, and from the continuity of $e_{\varepsilon}$ in $T$, we see that (5.5) is valid for some $T>T_{*}$; this contradicts the definition of $T_{*}$. Thus we conclude that $T_{*}=T_{0}$. In other words, we find that (5.5) is valid as long as the local solution exists, provided that $\varepsilon \in\left(0, \varepsilon_{0}\right]$. Now, with the help of (5.4), we see that $\sum_{|\alpha| \leq 2}\left\|\partial^{\alpha} u(t, \cdot)\right\|_{L^{\infty}\left(\mathbb{R}^{3}\right)}$ does not tend to infinity in finite time, and the local existence theorem implies the global existence of the solution for $\varepsilon \in\left(0, \varepsilon_{0}\right]$ (see Hörmander [7, Theorem 6.4.11] for instance). Since (5.5) holds for any $T>0$, we immediately get (2.17) and (2.18), and we also obtain (2.15) and (2.16) with the help of (5.4). This completes the proof.

The rest of this section is devoted to the proof of Proposition 5.1.

Proof of Proposition 5.1. We always assume $0<\varepsilon \leq \min \{1,1 / A\}$ in what follows, so that we have $0<A \varepsilon \leq 1$. The letter $C$ in this proof indicates various positive constants which may depend on $M_{0}$ and $R$, but are independent of $A, \varepsilon, T$, and $T_{0}$. The proof will be divided into several steps.

Step 1: Basic estimates. Assume that (5.5) is valid. Then (5.4) implies

$$
e_{\varepsilon}[u](T) \leq C_{0} \varepsilon+A \varepsilon^{2} \leq C \varepsilon,
$$


where $e_{\varepsilon}[u](T)$ is defined by replacing $\widetilde{u}=\left(\widetilde{v}^{\mathrm{T}}, \widetilde{w}^{\mathrm{T}}\right)^{\mathrm{T}}$ in $(5.3)$ with $u=\left(v^{\mathrm{T}}, w^{\mathrm{T}}\right)^{\mathrm{T}}$. As before, we write $r=|x|$ and $\omega=|x|^{-1} x$. Taking (3.2) and (3.3) into account, we obtain from (5.7) and Lemma 3.4 that

$$
\begin{aligned}
|\partial v(t, x)|_{m} & \leq C \varepsilon\langle t+r\rangle^{\lambda-1}\langle t-r\rangle^{-1} \\
|\partial w(t, x)|_{m+1} & \leq C \varepsilon\langle t+r\rangle^{-1}\langle t-r\rangle^{-1-\rho}
\end{aligned}
$$

for $(t, x) \in[0, T) \times \mathbb{R}^{3}$. Thus we have

$$
|\partial u(t, x)|_{m} \leq C \varepsilon\langle t+r\rangle^{\lambda-1}\langle t-r\rangle^{-1}
$$

Let $s$ be a nonnegative integer with $s \leq 2 m$. From (2.11), we have

$$
\begin{aligned}
& \left|F_{j}(u, \partial u)\right|_{s} \leq C\left(|\partial u|_{[s / 2]}|\partial u|_{s}+|w|_{[s / 2]}|\partial v|_{s}+|\partial v|_{[s / 2]}|w|_{s}\right), 1 \leq j \leq N^{\prime} \\
& \left|F_{j}(u, \partial u)\right|_{s} \leq C|\partial u|_{[s / 2]}|\partial u|_{s}, \quad N^{\prime}+1 \leq j \leq N
\end{aligned}
$$

We set

$$
\Lambda:=\left\{(t, x) \in \mathbb{R}_{+} \times \mathbb{R}^{3} ; r \geq t / 2 \geq 1\right\}
$$

and $\Lambda^{\mathrm{c}}:=\left(\mathbb{R}_{+} \times \mathbb{R}^{3}\right) \backslash \Lambda$. If $(t, x) \in \Lambda^{\mathrm{c}}$, then we have either $r<t / 2$ or $t<2$, and we obtain

$$
\langle t-r\rangle^{-1} \leq C\langle t+r\rangle^{-1}, \quad(t, x) \in \Lambda^{\mathrm{c}}
$$

Hence, using (5.7) and (5.10), we see from (5.11) and (5.12) that

$$
\begin{aligned}
& \sum_{j=1}^{N^{\prime}}\left|F_{j}(u, \partial u)\right|_{s} \leq C \varepsilon\left(\langle t+r\rangle^{-1-\rho}|\partial u|_{s}+\langle t+r\rangle^{\lambda-1} \frac{|w|_{s}}{\langle t-r\rangle}\right), \\
& \sum_{j=N^{\prime}+1}^{N}\left|F_{j}(u, \partial u)\right|_{s} \leq C \varepsilon\langle t+r\rangle^{\lambda-2}|\partial u|_{s}
\end{aligned}
$$

at $(t, x) \in \Lambda^{\mathrm{c}}$, where we have used $1+\rho<2-\lambda$.

For any nonnegative integer $s$ and any smooth function $\varphi=\varphi(\omega)$ on $S^{2}$, we have

$$
\sup _{(t, x) \in \Lambda} \sum_{|\alpha| \leq s}\left|\Gamma^{\alpha}\left(\varphi\left(r^{-1} x\right)\right)\right|<\infty .
$$

Hence, from (2.12) we get

$$
\left|G_{j}(\omega, u, \partial u)\right|_{s} \leq C\left(\left(|w|_{[s / 2]}+|\partial w|_{[s / 2]}\right)|\partial v|_{s}+|\partial v|_{[s / 2]}\left(|w|_{s}+|\partial w|_{s}\right)\right)
$$

in $\Lambda$ for $1 \leq j \leq N^{\prime}$. It follows from (5.7), (5.8), (15.9), and (5.15) that

$$
\sum_{j=1}^{N^{\prime}}\left|G_{j}\right|_{s} \leq C \varepsilon\langle t+r\rangle^{-1}\left(\langle t-r\rangle^{-\rho}|\partial v|_{s}+\langle t+r\rangle^{\lambda}\left(\frac{|w|_{s}}{\langle t-r\rangle}+|\partial w|_{s}\right)\right)
$$

at $(t, x) \in \Lambda$. Recalling (2.9) in Definition 2.1, and observing that

$$
|\partial u|_{[s / 2]}|u|_{s+1} \leq C\left(|\partial u|_{[s / 2]}|\Gamma u|_{s}+|\partial u|_{s}|u|_{[s / 2]+1}\right)
$$


we obtain from (2.13) that

$$
\sum_{j=1}^{N}\left|F_{j}-G_{j}\right|_{s} \leq C \varepsilon\langle t+r\rangle^{\lambda-2}\left(|\partial u|_{s}+\langle t-r\rangle^{-1}|\Gamma u|_{s}\right)
$$

at $(t, x) \in \Lambda$, where we have set $G_{j} \equiv 0$ for $N^{\prime}+1 \leq j \leq N$.

From (5.13), (5.16) and (5.17), we obtain

$$
\begin{aligned}
\sum_{j=1}^{N^{\prime}}\left|F_{j}\right|_{s} \leq & C \varepsilon\left(\langle t+r\rangle^{-1}\langle t-r\rangle^{-\rho}|\partial u|_{s}+\langle t+r\rangle^{\lambda-1}\left(\frac{|w|_{s}}{\langle t-r\rangle}+|\partial w|_{s}\right)\right) \\
& +C \varepsilon\langle t+r\rangle^{\lambda-2}\langle t-r\rangle^{-1}|\Gamma u|_{s}
\end{aligned}
$$

in $[0, T) \times \mathbb{R}^{3}$. By (5.14) and (5.17), we also obtain

$$
\sum_{j=N^{\prime}+1}^{N}\left|F_{j}\right|_{s} \leq C \varepsilon\langle t+r\rangle^{\lambda-2}\left(|\partial u|_{s}+\langle t-r\rangle^{-1}|\Gamma u|_{s}\right)
$$

in $[0, T) \times \mathbb{R}^{3}$.

Step 2: The energy estimates. We put

$$
I_{\varepsilon}(T)=\int_{0}^{T}\langle t\rangle^{\lambda-1}\left(\int_{\mathbb{R}^{3}} \frac{|u(t, x ; \varepsilon)|_{Z, 2 m}^{2}}{\langle t-r\rangle^{2}} d x\right)^{1 / 2} d t .
$$

Writing $\langle t\rangle^{\lambda-1}=\langle t\rangle^{3 \lambda-1}\langle t\rangle^{-2 \lambda}$, and using the Schwarz inequality, we obtain from (5.7) that

$$
I_{\varepsilon}(T) \leq\left(\int_{0}^{\infty}\langle t\rangle^{6 \lambda-2} d t\right)^{1 / 2}\left(\int_{0}^{T} \int_{\mathbb{R}^{3}} \frac{|u(t, x)|_{Z, 2 m}^{2}}{\langle t\rangle^{4 \lambda}\langle t-r\rangle^{2}} d x d t\right)^{1 / 2} \leq C \varepsilon
$$

where we have used $6 \lambda-2<-1$. Because of (5.2), we can use Lemma 3.5 to obtain

$$
\left(\int_{\mathbb{R}^{3}} \frac{|w(t, x)|_{s}^{2}}{\langle t-r\rangle^{2}} d x\right)^{1 / 2} \leq C\|\partial w(t, \cdot)\|_{s}
$$

Lemma 3.6 implies

$$
\begin{aligned}
\langle t+r\rangle^{\lambda-2}\langle t & -r\rangle^{-1}|\Gamma u|_{s} \\
& \leq C\langle t+r\rangle^{\lambda-1}\left(\langle t-r\rangle^{-1}|u|_{Z, s}+\langle t+r\rangle^{-1}|\partial u|_{s}\right) .
\end{aligned}
$$

Let $\mu \geq 0$. In view of (5.18), (5.21), and (5.22) with $s=2 m$, we obtain from (5.7) and (5.20) that

$$
\begin{aligned}
\sum_{j=1}^{N^{\prime}} \int_{0}^{t}\langle\tau\rangle^{-\mu}\left\|F_{j}(\tau)\right\|_{2 m} d \tau \leq & C \varepsilon \int_{0}^{t}\langle\tau\rangle^{-1-\mu}\left(\|\partial u(\tau)\|_{2 m}+\langle\tau\rangle^{\lambda}\|\partial w(\tau)\|_{2 m}\right) d \tau \\
& +C \varepsilon I_{\varepsilon}(T) \\
\leq & C \varepsilon^{2} \int_{0}^{t}\langle\tau\rangle^{\lambda-1-\mu} d \tau+C \varepsilon^{2}, \quad 0 \leq t \leq T
\end{aligned}
$$


Similarly, using (5.19) with $s=2 m$ instead of (5.18), we obtain

$$
\begin{aligned}
\sum_{j=N^{\prime}+1}^{N} \int_{0}^{t}\left\|F_{j}(\tau)\right\|_{2 m} d \tau & \leq C \varepsilon\left(\int_{0}^{t}\langle\tau\rangle^{\lambda-2}\|\partial u(\tau)\|_{2 m} d \tau+I_{\varepsilon}(T)\right) \\
& \leq C \varepsilon^{2} \int_{0}^{t}\langle\tau\rangle^{2 \lambda-2} d \tau+C \varepsilon^{2} \leq C \varepsilon^{2}, \quad 0 \leq t<T .
\end{aligned}
$$

From (1.1), we have

$$
\square \widetilde{u}_{j}=F_{j}(u, \partial u), \quad 1 \leq j \leq N,
$$

and $\widetilde{u}(0, x)=\left(\partial_{t} \widetilde{u}\right)(0, x) \equiv 0$. From (3.1) and (15.25) we get

$$
\square\left(\Gamma^{\alpha} \widetilde{u}_{j}\right)=\left(\Gamma_{0}+2\right)^{\alpha_{0}} \Gamma_{1}^{\alpha_{1}} \cdots \Gamma_{10}^{\alpha_{10}}\left(F_{j}(u, \partial u)\right), \quad 1 \leq j \leq N
$$

for any multi-index $\alpha=\left(\alpha_{0}, \alpha_{1}, \ldots, \alpha_{10}\right)$. We also get

$$
\left\|\left(\Gamma^{\alpha} \widetilde{u}\right)(0)\right\|_{L^{2}\left(\mathbb{R}^{3}\right)}+\left\|\left(\partial_{t} \Gamma^{\alpha} \widetilde{u}\right)(0)\right\|_{L^{2}\left(\mathbb{R}^{3}\right)} \leq C_{\alpha} \varepsilon^{2}
$$

with some positive constant $C_{\alpha}$. Applying Lemma 3.8 with $\mu=2 \lambda$ to (5.26) for $|\alpha| \leq 2 m$, and using (5.23) and (5.24), we get

$$
\begin{aligned}
\left(\int_{0}^{T} \int_{\mathbb{R}^{3}} \frac{|\widetilde{u}(\tau, x)|_{Z, 2 m}^{2}}{\langle\tau\rangle^{4 \lambda}\langle\tau-r\rangle^{2}} d x d \tau\right)^{1 / 2} & \leq C \varepsilon^{2}+C \int_{0}^{T}\langle\tau\rangle^{-2 \lambda}\|F(u, \partial u)(\tau, \cdot)\|_{2 m} d \tau \\
& \leq C \varepsilon^{2}\left(1+\int_{0}^{\infty}\langle\tau\rangle^{-\lambda-1} d \tau\right) \leq C \varepsilon^{2}
\end{aligned}
$$

Applying Lemma 3.8 with $\mu=0$ to (5.26) for $1 \leq j \leq N^{\prime}$ and $|\alpha| \leq 2 m$, and using (5.23), we obtain

$$
\sum_{j=1}^{N^{\prime}}\left\|\partial \widetilde{v}_{j}(t, \cdot)\right\|_{2 m} \leq C \varepsilon^{2}+C \sum_{j=1}^{N^{\prime}} \int_{0}^{t}\left\|F_{j}(u, \partial u)(\tau, \cdot)\right\|_{2 m} d \tau \leq C \varepsilon^{2}\langle t\rangle^{\lambda}
$$

for $0 \leq t<T$, which implies

$$
\sup _{0 \leq t<T}(1+t)^{-\lambda}\|\partial \widetilde{v}(t, \cdot)\|_{2 m} \leq C \varepsilon^{2}
$$

Similarly, applying Lemma 3.8 to (5.26) for $N^{\prime}+1 \leq j \leq N$, and using (5.24) instead of (5.23), we obtain

$$
\sup _{0 \leq t<T}\|\partial \widetilde{w}(t, \cdot)\|_{2 m} \leq C \varepsilon^{2} .
$$

Step 3: Decay estimates for generalized derivatives of higher order. Now we turn our attention to the decay estimates. By (5.7) and Lemma 3.1, we have

$$
|\partial u(t, x)|_{2 m-2} \leq C \varepsilon\langle t+r\rangle^{\lambda-1}\langle t-r\rangle^{-1 / 2}, \quad(t, x) \in[0, T) \times \mathbb{R}^{3} .
$$

It follows from (5.10), (5.30), and (5.12) with $s=2 m-2$ that

$$
\left|F_{j}(u, \partial u)\right|_{2 m-2} \leq C \varepsilon^{2}\langle t+r\rangle^{2 \lambda-2}\langle t-r\rangle^{-3 / 2}, \quad N^{\prime}+1 \leq j \leq N .
$$


Hence, applying Lemma 3.9 (with $\mu=1-2 \lambda$ ) to (5.26) for $N^{\prime}+1 \leq j \leq N$ and $|\alpha| \leq 2 m-2$, and using (5.4), we obtain

$$
\langle t+r\rangle^{1-2 \lambda}|w(t, x)|_{2 m-2} \leq C \varepsilon, \quad(t, x) \in[0, T) \times \mathbb{R}^{3} .
$$

Therefore Lemma 3.4 yields

$$
\langle t+r\rangle^{1-2 \lambda}\langle t-r\rangle|\partial w(t, x)|_{2 m-3} \leq C \varepsilon, \quad(t, x) \in[0, T) \times \mathbb{R}^{3} .
$$

Using (5.7), (5.10), (5.30), and (5.31) to evaluate the right-hand side of (5.11) with $s=2 m-2$, we get

$$
\begin{aligned}
\left|F_{j}(u, \partial u)\right|_{2 m-2} & \leq C \varepsilon^{2}\left(\langle t+r\rangle^{2 \lambda-2}\langle t-r\rangle^{-\rho-(1 / 2)}+\langle t+r\rangle^{3 \lambda-2}\langle t-r\rangle^{-1}\right) \\
& \leq C \varepsilon^{2}\langle t+r\rangle^{4 \lambda-2}\langle t-r\rangle^{-1-\lambda}, \quad 1 \leq j \leq N^{\prime} .
\end{aligned}
$$

Similarly to the derivation of (5.31) and (5.32), Lemmas 3.9 and 3.4 lead to

$$
\langle t+r\rangle^{1-4 \lambda}\left(|v(t, x)|_{2 m-2}+\langle t-r\rangle|\partial v(t, x)|_{2 m-3}\right) \leq C \varepsilon
$$

for $(t, x) \in[0, T) \times \mathbb{R}^{3}$.

Step 4: Decay estimates for generalized derivatives of lower order. By (5.31) and (5.33) we get

$$
|\Gamma u(t, x)|_{2 m-3} \leq C|u(t, x)|_{2 m-2} \leq C \varepsilon\langle t+r\rangle^{4 \lambda-1} .
$$

Using (5.32), (5.33), and (5.34) to estimate the right-hand side of (5.19) with $s=2 m-3$, we obtain

$$
\sum_{j=N^{\prime}+1}^{N}\left|F_{j}(u, \partial u)\right|_{2 m-3} \leq C \varepsilon^{2}\langle t+r\rangle^{5 \lambda-3}\langle t-r\rangle^{-1} \leq C \varepsilon^{2}\langle t+r\rangle^{6 \lambda-3}\langle t-r\rangle^{-1-\lambda}
$$

Hence applying Lemma 3.9 with $\mu=1+\rho$, and then using Lemma 3.4, we get

$$
\langle t+r\rangle\langle t-r\rangle^{\rho}\left(|\widetilde{w}(t, x)|_{2 m-3}+\langle t-r\rangle|\partial \widetilde{w}(t, x)|_{2 m-4}\right) \leq C \varepsilon^{2}
$$

for $(t, x) \in[0, T) \times \mathbb{R}^{3}$, because $1 / 2<\rho \leq 1-6 \lambda$. Now (5.4) leads to

$$
\langle t+r\rangle\langle t-r\rangle^{\rho}\left(|w(t, x)|_{2 m-3}+\langle t-r\rangle|\partial w(t, x)|_{2 m-4}\right) \leq C \varepsilon .
$$

Using (5.30), (5.36), and (5.34) to estimate each term on the right-hand side of (5.18) with $s=2 m-4$, we obtain

$$
\begin{aligned}
\sum_{j=1}^{N^{\prime}}\left|F_{j}(u, \partial u)\right|_{2 m-4} \leq & C \varepsilon^{2}\left(\langle t+r\rangle^{\lambda-2}\langle t-r\rangle^{-\rho-(1 / 2)}+\langle t+r\rangle^{\lambda-2}\langle t-r\rangle^{-\rho-1}\right) \\
& +C \varepsilon^{2}\langle t+r\rangle^{5 \lambda-3}\langle t-r\rangle^{-1} \\
\leq & C \varepsilon^{2}\langle t+r\rangle^{\lambda-2}\langle t-r\rangle^{-\rho-(1 / 2)},
\end{aligned}
$$

where we have used $\langle t+r\rangle^{5 \lambda-3}\langle t-r\rangle^{-1} \leq\langle t+r\rangle^{\lambda-2}\langle t-r\rangle^{4 \lambda-2}$ and $\rho+(1 / 2)<$ $2-4 \lambda$. Since $\rho+(1 / 2)>1$, Lemma 3.9 with $\mu=1-\lambda$ implies

$$
\langle t+r\rangle^{1-\lambda}|\widetilde{v}(t, x)|_{2 m-4} \leq C \varepsilon^{2}, \quad(t, x) \in[0, T) \times \mathbb{R}^{3} .
$$


Step 5: Conclusion. Since we have $m \leq 2 m-5$, it follows from (5.27), (5.28), (5.29), (5.35), and (5.37) that

$$
e_{\varepsilon}[\widetilde{u}](T) \leq C_{1} \varepsilon^{2}
$$

with an appropriate positive constant $C_{1}=C_{1}\left(M_{0}, R\right)$, which is independent of $A$, $\varepsilon, T$, and $T_{0}$. Finally, if we choose $A_{0}=2 C_{1}$, (5.38) immediately implies (5.6) for $A \geq A_{0}$. This completes the proof.

\section{Proof of Theorem 2.6}

We start this section with a fundamental observation. We set

$$
\Lambda_{0}:=\{(t, r) \in[0, \infty) \times[0, \infty) ; r \geq t / 2 \geq 1\}
$$

and we put $t_{0}(\sigma)=\max \{2,-2 \sigma\}$ for $\sigma \in \mathbb{R}$ so that

$$
\bigcup_{\sigma \in \mathbb{R}}\left\{(t, t+\sigma) ; t \geq t_{0}(\sigma)\right\}=\Lambda_{0}
$$

We take a smooth and non-increasing function $t_{1}=t_{1}(\sigma)$ such that $t_{1}(\sigma)=-2 \sigma$ for $\sigma \leq-2, t_{1}(\sigma)=2$ for $\sigma \geq 0$, and $t_{0}(\sigma) \leq t_{1}(\sigma) \leq 2-\sigma$ for $-2<\sigma<0$. We define $r_{1}(\sigma):=t_{1}(\sigma)+\sigma$. Note that we have

$$
1+|\sigma| \leq 1+t_{1}(\sigma)+r_{1}(\sigma)=1+2 t_{1}(\sigma)+\sigma \leq 5(1+|\sigma|), \quad \sigma \in \mathbb{R} .
$$

Lemma 6.1. Suppose that $\mu>1$ and $\kappa \geq 0$.

Let $\varphi=\varphi(t, r, \omega)$ be a $C^{1}$-function of $(t, r) \in \Lambda_{0}$ and $\omega \in S^{2}$, satisfying

$$
\partial_{+} \varphi(t, r, \omega)=h(t, r, \omega), \quad(t, r) \in \Lambda_{0}, \omega \in S^{2},
$$

where $\partial_{+}=\partial_{t}+\partial_{r}$. Assume that there is a positive constant $C_{0}$ such that

$$
|h(t, r, \omega)| \leq C_{0}(1+t+r)^{-\mu}(1+|t-r|)^{-\kappa}, \quad(t, r) \in \Lambda_{0}, \omega \in S^{2} .
$$

Then, putting

$$
\Phi(\sigma, \omega)=\varphi\left(t_{1}(\sigma), r_{1}(\sigma), \omega\right)+\int_{t_{1}(\sigma)}^{\infty} h(\tau, \tau+\sigma, \omega) d \tau, \quad(\sigma, \omega) \in \mathbb{R} \times S^{2},
$$

we have

$$
|\varphi(t, t+\sigma, \omega)-\Phi(\sigma, \omega)| \leq \frac{C_{0}}{2(\mu-1)}(1+2 t+\sigma)^{-(\mu-1)}(1+|\sigma|)^{-\kappa}
$$

for $t \geq t_{0}(\sigma)$ and $(\sigma, \omega) \in \mathbb{R} \times S^{2}$.

Proof. Observe that $\Phi$ is well-defined because of the assumption (6.3).

It is easy to see that

$$
\varphi(t, t+\sigma, \omega)=\varphi\left(t_{1}(\sigma), r_{1}(\sigma), \omega\right)+\int_{t_{1}(\sigma)}^{t} h(\tau, \tau+\sigma, \omega) d \tau
$$


for $t \geq t_{0}(\sigma)$ and $(\sigma, \omega) \in \mathbb{R} \times S^{2}$. Hence (6.3) and (6.4) yield

$$
\begin{aligned}
|\varphi(t, t+\sigma, \omega)-\Phi(\sigma, \omega)| & \leq \int_{t}^{\infty}|h(\tau, \tau+\sigma, \omega)| d \tau \\
& \leq C_{0}(1+|\sigma|)^{-\kappa} \int_{t}^{\infty}(1+2 \tau+\sigma)^{-\mu} d \tau
\end{aligned}
$$

for $t \geq t_{0}(\sigma)$ and $(\sigma, \omega) \in \mathbb{R} \times S^{2}$; thus the direct calculation leads to (6.5). This completes the proof.

From (6.4), it is easy to see the following:

Lemma 6.2. Let $\varphi, h$, and $\Phi$ be as in Lemma 6.1. Assume in addition that $h$ is $a C^{1}$-function of $(t, r) \in \Lambda_{0}$ and $\omega \in S^{2}$. If there exist two constants $\mu^{\prime}>1$ and $C>0$ such that

$$
\sum_{p+|\alpha|=1}\left|\partial_{r}^{p} \Omega^{\alpha} h(t, r, \omega)\right| \leq C(1+t+r)^{-\mu^{\prime}}, \quad(t, r) \in \Lambda_{0}, \omega \in S^{2},
$$

then we have $\Phi \in C^{1}\left(\mathbb{R} \times S^{2}\right)$, where $\Omega$ is regarded as a differential operator on $S^{2}$.

Let us start the proof of Theorem 2.6. Suppose that the assumptions in Theorem 2.6 are satisfied, and let $u=\left(v^{\mathrm{T}}, w^{\mathrm{T}}\right)^{\mathrm{T}}$ be the global solution to (1.1)-(2.8) with $(f, g) \in D$. We define $u^{0}, v^{0}, w^{0}, \widetilde{u}, \widetilde{v}$, and $\widetilde{w}$ as in the previous section. Recall that, by Theorem 2.5 and Lemma 3.4, we have

$$
\begin{aligned}
& |v(t, x)|_{2}+\varepsilon^{-1}|\widetilde{v}(t, x)|_{2}+(1+|t-| x||)\left(|\partial v(t, x)|_{1}+\varepsilon^{-1}|\partial \widetilde{v}(t, x)|_{1}\right) \\
& \quad \leq C \varepsilon(1+t+|x|)^{\lambda-1}, \\
& \quad|w(t, x)|_{3}+\varepsilon^{-1}|\widetilde{w}(t, x)|_{3}+(1+|t-| x||)\left(|\partial w(t, x)|_{2}+\varepsilon^{-1}|\partial \widetilde{w}(t, x)|_{2}\right) \\
& \quad \leq C \varepsilon(1+t+|x|)^{-1}(1+|t-| x||)^{-\rho}
\end{aligned}
$$

for any $(t, x) \in[0, \infty) \times \mathbb{R}^{3}$ and $\varepsilon \in\left(0, \varepsilon_{0}\right]$, where $\lambda(\in(0,1 / 20)), \rho(\in(1 / 2,1-6 \lambda])$, and $\varepsilon_{0}(>0)$ are from Theorem 2.5 , and $C$ is a positive constant. Remember also that we have (5.1) and (5.2) for some $R>0$.

Let $0<\varepsilon \leq \varepsilon_{0}$. We define $U_{j}^{0} \in C_{0}^{\infty}\left(\mathbb{R} \times S^{2}\right)$ by

$$
U_{j}^{0}(\sigma, \omega)=\mathcal{F}_{0}\left[f_{j}(\cdot ; \varepsilon), g_{j}(\cdot ; \varepsilon)\right](\sigma, \omega), \quad(\sigma, \omega) \in \mathbb{R} \times S^{2}
$$

for $1 \leq j \leq N$, where $\mathcal{F}_{0}$ is given by (1.13). Recall that (15.1) implies $u_{j}^{0}(t, r \omega)=0$ for $|r-t| \geq R$, and

$$
U_{j}^{0}(\sigma, \omega)=0, \quad|\sigma| \geq R
$$

(cf. (3.22) ). Therefore (1.15) implies that for any $\kappa \geq 0$, there exists a positive constant $C_{\kappa}$ such that

$$
\begin{gathered}
\left|r u_{j}^{0}(t, r \omega)-U_{j}^{0}(r-t, \omega)\right|+\sum_{a=0}^{3}\left|r\left(\partial_{a} u_{j}^{0}\right)(t, r \omega)-\omega_{a}\left(\partial_{\sigma} U_{j}^{0}\right)(r-t, \omega)\right| \\
\leq C_{\kappa}(1+t+r)^{-1}(1+|t-r|)^{-\kappa}, \quad 1 \leq j \leq N
\end{gathered}
$$


for $(t, r) \in \Lambda_{0}$ and $\omega \in S^{2}$. Indeed we have $(1+|t-r|)^{-\kappa} \geq(1+R)^{-\kappa}$ in the support of the functions on the left-hand side of (6.10). We put $V_{j}^{0}=U_{j}^{0}$ for $1 \leq j \leq N^{\prime}$, and $W_{k}^{0}=U_{N^{\prime}+k}^{0}$ for $1 \leq k \leq N^{\prime \prime}$.

For any smooth function $\psi$, we have

$$
r \square \psi(t, x)=\partial_{+} \partial_{-}(r \psi(t, x))-r^{-1}\left(\Delta_{\omega} \psi\right)(t, x), \quad(t, x) \in[0, \infty) \times \mathbb{R}^{3},
$$

where $r=|x|$, and $\Delta_{\omega}=\sum_{k=1}^{3} \Omega_{k}^{2}$. Hence by (5.25) we have

$$
\partial_{+} \partial_{-}\left(r \widetilde{u}_{j}(t, r \omega)\right)=H_{j}(t, r, \omega), \quad 1 \leq j \leq N
$$

where $H_{j}$ is given by

$$
H_{j}(t, r, \omega)=r F_{j}(u(t, r \omega), \partial u(t, r \omega))+r^{-1}\left(\Delta_{\omega} \widetilde{u}_{j}\right)(t, r \omega) .
$$

We always suppose $(t, r) \in \Lambda_{0}$ (namely $r \geq t / 2 \geq 1$ ), and $\omega \in S^{2}$ from now on. Then we have $r^{-1} \leq 4(1+t+r)^{-1}$. In what follows, $C$ denotes various positive constants which are independent of $(t, r) \in \Lambda_{0}, \omega \in S^{2}$, and $\varepsilon \in\left(0, \varepsilon_{0}\right]$.

Equations (6.7) and (6.8) yield

$$
\begin{aligned}
\left|r^{-1}\left(\Delta_{\omega} \widetilde{v}_{j}\right)(t, r \omega)\right| & \leq C \varepsilon^{2}(1+t+r)^{\lambda-2}, \quad 1 \leq j \leq N^{\prime}, \\
\left|r^{-1}\left(\Delta_{\omega} \widetilde{w}_{k}\right)(t, r \omega)\right|_{1} & \leq C \varepsilon^{2}(1+t+r)^{-2}(1+|t-r|)^{-\rho}, \quad 1 \leq k \leq N^{\prime \prime} .
\end{aligned}
$$

Because of (2.9) and (2.13) we obtain

$$
\begin{aligned}
\left|r F_{j}(u, \partial u)-r G_{j}(\omega, u, \partial u)\right|_{1} & \leq C|u|_{2}|\partial u|_{1} \\
& \leq C \varepsilon^{2}(1+t+r)^{2 \lambda-2}(1+|t-r|)^{-1}
\end{aligned}
$$

at $(t, x)=(t, r \omega)$ for $1 \leq j \leq N$, where $G_{j}$ for $1 \leq j \leq N^{\prime}$ is from Condition 2.2. and we have set $G_{j}(\omega, u, \partial u) \equiv 0$ for $N^{\prime}+1 \leq j \leq N$ as before.

We first construct the standard asymptotic profile $W$ for $w$, and then the modified asymptotic profile $V$ for $v$.

Step 1: Construction of $W$. From (6.15) and (6.16), we get

$$
\left|H_{N^{\prime}+k}(t, r, \omega)\right|_{1} \leq C \varepsilon^{2}(1+t+r)^{2 \lambda-2}(1+|t-r|)^{-\rho}, \quad 1 \leq k \leq N^{\prime \prime} .
$$

We put

$$
\widetilde{w}_{k,-}(t, r, \omega):=\partial_{-}\left(r \widetilde{w}_{k}(t, r \omega)\right)\left(=\partial_{-}\left(r \widetilde{u}_{N^{\prime}+k}(t, r \omega)\right)\right), \quad 1 \leq k \leq N^{\prime \prime} .
$$

Equation (6.8) leads to

$$
\left|\widetilde{w}_{k,-}(t, r, \omega)\right| \leq C \varepsilon^{2}(1+|t-r|)^{-1-\rho}, \quad 1 \leq k \leq N^{\prime \prime}
$$

Because of (6.12) and (6.17), Lemma 6.1 implies

$$
\left|\widetilde{w}_{k,-}(t, t+\sigma, \omega)-\varepsilon \widetilde{W}_{k,-}(\sigma, \omega)\right| \leq C \varepsilon^{2}(1+2 t+\sigma)^{2 \lambda-1}(1+|\sigma|)^{-\rho}
$$

for $1 \leq k \leq N^{\prime \prime}, t \geq t_{0}(\sigma)$, and $(\sigma, \omega) \in \mathbb{R} \times S^{2}$, where $\widetilde{W}_{k,-}$ is defined by

$$
\varepsilon \widetilde{W}_{k,-}(\sigma, \omega)=\widetilde{w}_{k,-}\left(t_{1}(\sigma), r_{1}(\sigma), \omega\right)+\int_{t_{1}(\sigma)}^{\infty} H_{N^{\prime}+k}(\tau, \tau+\sigma, \omega) d \tau \text {. }
$$


Note that we have $\widetilde{W}_{k,-} \in C^{1}\left(\mathbb{R} \times S^{2}\right)$, because of Lemma 6.2 and (6.17). By (6.18) and (6.19) we get

$$
\left|\widetilde{W}_{k,-}(\sigma, \omega)\right| \leq C \varepsilon\left((1+|\sigma|)^{-1-\rho}+(1+2 t+\sigma)^{2 \lambda-1}(1+|\sigma|)^{-\rho}\right) .
$$

Therefore, taking the limit as $t \rightarrow \infty$, we obtain

$$
\left|\widetilde{W}_{k,-}(\sigma, \omega)\right| \leq C \varepsilon(1+|\sigma|)^{-1-\rho}, \quad 1 \leq k \leq N^{\prime \prime},(\sigma, \omega) \in \mathbb{R} \times S^{2} .
$$

Furthermore, since (5.2) yields $\widetilde{w}_{k,-}(t, t+\sigma, \omega)=H_{N^{\prime}+k}(t, t+\sigma, \omega)=0$ when $\sigma \geq R,(6.20)$ implies

$$
\widetilde{W}_{k,-}(\sigma, \omega)=0 \quad \text { for } 1 \leq k \leq N^{\prime \prime}, \sigma \geq R \text {, and } \omega \in S^{2} .
$$

For $1 \leq k \leq N^{\prime \prime}$, we define

$$
\widetilde{W}_{k}(\sigma, \omega)=\frac{1}{2} \int_{\sigma}^{\infty} \widetilde{W}_{k,-}(\tau, \omega) d \tau, \quad(\sigma, \omega) \in \mathbb{R} \times S^{2},
$$

so that we have

$$
-2 \partial_{\sigma} \widetilde{W}_{k}(\sigma, \omega)=\widetilde{W}_{k,-}(\sigma, \omega), \quad(\sigma, \omega) \in \mathbb{R} \times S^{2} .
$$

By (6.22) and (6.23) we get

$$
\widetilde{W}_{k}(\sigma, \omega)=0, \quad \sigma \geq R, \omega \in S^{2} .
$$

Using (3.10) and (6.8), we obtain from (6.19) and (6.24) that

$$
\begin{aligned}
& \sum_{a=0}^{3}\left|r\left(\partial_{a} \widetilde{w}_{k}\right)(t, r \omega)-\varepsilon \omega_{a}\left(\partial_{\sigma} \widetilde{W}_{k}\right)(r-t, \omega)\right| \\
& \quad \leq C \varepsilon^{2}(1+t+r)^{2 \lambda-1}(1+|t-r|)^{-\rho}, \quad 1 \leq k \leq N^{\prime \prime} .
\end{aligned}
$$

We set

$$
\widetilde{w}_{k}^{*}(t, r, \omega)=r \widetilde{w}_{k}(t, r \omega), \quad 1 \leq k \leq N^{\prime \prime},
$$

so that we have $\partial_{-} \widetilde{w}_{k}^{*}(t, r, \omega)=\widetilde{w}_{k,-}(t, r, \omega)$. Now we are going to prove

$$
\left|\widetilde{w}_{k}^{*}(t, t+\sigma, \omega)-\varepsilon \widetilde{W}_{k}(\sigma, \omega)\right| \leq C \varepsilon^{2}(1+2 t+\sigma)^{2 \lambda-1}(1+|\sigma|)^{1-\rho}
$$

for $t \geq t_{0}(\sigma)$ and $(\sigma, \omega) \in \mathbb{R} \times S^{2}$. If $\sigma>R$, then (6.27) is trivial because of (5.2) and (6.25). To treat the case where $\sigma \leq R$, we put

$$
\begin{aligned}
& D_{1}=\left\{(t, \sigma) ; t \geq \max \left\{t_{0}(\sigma), 2+(R-\sigma) / 2\right\}, \sigma \leq R\right\}, \\
& D_{2}=\left\{(t, \sigma) ; t_{0}(\sigma) \leq t \leq 2+(R-\sigma) / 2, \sigma \leq R\right\},
\end{aligned}
$$

so that we have $\left\{(t, \sigma) ; t \geq t_{0}(\sigma), \sigma \leq R\right\}=D_{1} \cup D_{2}$. If $(t, \sigma) \in D_{1}$, then observing that we have

$$
\widetilde{w}_{k}^{*}(t-(R-\sigma) / 2, t+(R+\sigma) / 2, \omega)=\widetilde{W}_{k}(R, \omega)=0, \quad 1 \leq k \leq N^{\prime \prime}
$$


(cf. (5.2) and (6.25) ), and remembering (6.24), we get

$$
\begin{aligned}
& \widetilde{w}_{k}^{*}(t, t+\sigma, \omega)-\varepsilon \widetilde{W}_{k}(\sigma, \omega) \\
& =\int_{t-(R-\sigma) / 2}^{t} \partial_{\tau}\left\{\widetilde{w}_{k}^{*}(\tau, 2 t+\sigma-\tau, \omega)-\varepsilon \widetilde{W}_{k}(2 t+\sigma-2 \tau, \omega)\right\} d \tau \\
& =\int_{t-(R-\sigma) / 2}^{t}\left\{\widetilde{w}_{k,-}(\tau, 2 t+\sigma-\tau, \omega)-\varepsilon \widetilde{W}_{k,-}(2 t+\sigma-2 \tau, \omega)\right\} d \tau .
\end{aligned}
$$

Since we have $(\tau, 2 t+\sigma-\tau) \in \Lambda_{0}$ for $t-(R-\sigma) / 2 \leq \tau \leq t$ when $(t, \sigma) \in D_{1}$, we obtain from (6.19) that

$$
\begin{aligned}
& \left|\widetilde{w}_{k}^{*}(t, t+\sigma, \omega)-\varepsilon \widetilde{W}_{k}(\sigma, \omega)\right| \\
& \quad \leq C \varepsilon^{2}(1+2 t+\sigma)^{2 \lambda-1} \int_{t-(R-\sigma) / 2}^{t}(1+|2 t+\sigma-2 \tau|)^{-\rho} d \tau \\
& \quad \leq C \varepsilon^{2}(1+2 t+\sigma)^{2 \lambda-1}(1+|\sigma|)^{1-\rho}, \quad(t, \sigma) \in D_{1}, \omega \in S^{2} .
\end{aligned}
$$

Because $D_{2}$ is a bounded set, it follows from (6.8), (6.21), and (6.23) that

$$
\left|\widetilde{w}_{k}^{*}(t, t+\sigma, \omega)\right|+\left|\varepsilon \widetilde{W}_{k}(\sigma, \omega)\right| \leq C \varepsilon^{2} \leq C \varepsilon^{2}(1+2 t+\sigma)^{2 \lambda-1}(1+|\sigma|)^{1-\rho}
$$

for $(t, \sigma) \in D_{2}$ and $\omega \in S^{2}$. Now (6.27) for $\sigma \leq R$ follows from (6.28) and (6.29).

Similarly to (6.21), we obtain from (6.8) and (6.27) that

$$
\left|\widetilde{W}_{k}(\sigma, \omega)\right| \leq C \varepsilon(1+|\sigma|)^{-\rho}, \quad(\sigma, \omega) \in \mathbb{R} \times S^{2} .
$$

Finally we define

$$
W_{k}(\sigma, \omega)=W_{k}^{0}(\sigma, \omega)+\widetilde{W}_{k}(\sigma, \omega), \quad(\sigma, \omega) \in \mathbb{R} \times S^{2}
$$

for $1 \leq k \leq N^{\prime \prime}$. Then, from (6.10), (6.26), and (6.27), we find that (2.22) and (2.23) are true. (2.25) follows from (6.21) and (6.30).

Note that we have (2.27) by (2.25) and (6.9) . Notice also that we have $\partial_{\sigma}^{p} W_{k} \in$ $C^{1}\left(\mathbb{R} \times S^{2}\right)$ for $1 \leq k \leq N^{\prime \prime}$ and $p=0,1$.

Step 2: Construction of $V$. We define

$$
\begin{aligned}
v_{-}^{\dagger}(t, r, \omega) & =\left(v_{1,-}^{\dagger}(t, r, \omega), \ldots, v_{N^{\prime},-}^{\dagger}(t, r, \omega)\right)^{\mathrm{T}} \\
& :=e^{-\Theta_{\varepsilon}(t, r-t, \omega)} \partial_{-}(r v(t, r \omega)),
\end{aligned}
$$

where

$$
\boldsymbol{\Theta}_{\varepsilon}(t, \sigma, \omega):=(\varepsilon \log t) \mathbf{A}[W](\sigma, \omega),
$$

and $\mathbf{A}[W](\sigma, \omega)$ is given by (2.19) -(2.20) with $W=W(\sigma, \omega)=\left(W_{k}(\sigma, \omega)\right)_{1<k<N^{\prime \prime}}^{\mathrm{T}}$ just having been constructed. Since $\partial_{+}\left(\boldsymbol{\Theta}_{\varepsilon}(t, r-t, \omega)\right)\left(=\varepsilon t^{-1} \mathbf{A}[W](r-t, \omega)\right)$ commutes with $\boldsymbol{\Theta}_{\varepsilon}(t, r-t, \omega)$, we have

$$
\partial_{+}\left(e^{-\boldsymbol{\Theta}_{\varepsilon}(t, r-t, \omega)}\right)=-\frac{\varepsilon}{t} e^{-\boldsymbol{\Theta}_{\varepsilon}(t, r-t, \omega)} \mathbf{A}[W](r-t, \omega) .
$$


Hence we get

$$
\partial_{+} v_{-}^{\dagger}(t, r, \omega)=e^{-\Theta_{\varepsilon}(t, r-t, \omega)}\left\{\partial_{+} \partial_{-}(r v(t, r \omega))-\frac{\varepsilon}{t} \mathbf{A}[W](r-t, \omega) \partial_{-}(r v(t, r \omega))\right\},
$$

and from (6.11) and (6.12) we obtain

$$
\partial_{+} v_{j,-}^{\dagger}(t, r, \omega)=\Psi_{j}(t, r, \omega), \quad 1 \leq j \leq N^{\prime}
$$

where

$$
\begin{aligned}
\Psi(t, r, \omega) & =\left(\Psi_{1}(t, r, \omega), \ldots, \Psi_{N^{\prime}}(t, r, \omega)\right)^{\mathrm{T}} \\
& =e^{-\Theta_{\varepsilon}(t, r-t, \omega)}\left(H^{\#}(t, r, \omega)+\varepsilon r^{-1}\left(\Delta_{\omega} v^{0}\right)(t, r \omega)\right), \\
H^{\#}(t, r, \omega) & =\left(H_{1}^{\#}(t, r, \omega), \ldots, H_{N^{\prime}}^{\#}(t, r, \omega)\right)^{\mathrm{T}} \\
& =\left(H_{1}(t, r, \omega), \ldots, H_{N^{\prime}}(t, r, \omega)\right)^{\mathrm{T}}-\frac{\varepsilon}{t} \mathbf{A}[W](r-t, \omega) \partial_{-}(r v(t, r \omega)) .
\end{aligned}
$$

Here $H_{j}(t, r, \omega)$ for $1 \leq j \leq N^{\prime}$ is given by (6.13).

By (2.19), (2.20), and (2.27) we obtain

$$
\|\mathbf{A}[W](\sigma, \omega)\| \leq C\left(|W(\sigma, \omega)|+\left|\partial_{\sigma} W(\sigma, \omega)\right|\right) \leq C(1+|\sigma|)^{-\rho},
$$

where $\|\mathbf{B}\|$ denotes the operator norm for a matrix $\mathbf{B}$. Hence we get $\left\|\boldsymbol{\Theta}_{\varepsilon}(t, \sigma, \omega)\right\| \leq$ $C \varepsilon \log t$, which leads to

$$
\left\|e^{ \pm \boldsymbol{\Theta}_{\varepsilon}(t, \sigma, \omega)}\right\| \leq e^{\left\|\boldsymbol{\Theta}_{\varepsilon}(t, \sigma, \omega)\right\|} \leq e^{C \varepsilon \log t} \leq t^{C \varepsilon}
$$

and

$$
\left\|e^{-\boldsymbol{\Theta}_{\varepsilon}(t, \sigma, \omega)}-\mathbf{I}\right\| \leq e^{\left\|\boldsymbol{\Theta}_{\varepsilon}(t, \sigma, \omega)\right\|}-1 \leq e^{C \varepsilon \log t}-1 \leq C \varepsilon t^{C \varepsilon+\lambda}
$$

for $t>0$ and $(\sigma, \omega) \in \mathbb{R} \times S^{2}$. Here, in order to obtain (6.37), we have used the inequality $e^{\tau}-1 \leq \tau e^{\tau}$ that is valid for $\tau \geq 0$, and the inequality $\log t \leq C_{\lambda} t^{\lambda}$ that is valid for $t \geq 1$ with some positive constant $C_{\lambda}$ depending only on $\lambda>0$.

From (5.4) we get

$$
r^{-1}\left|\left(\Delta_{\omega} v^{0}\right)(t, r \omega)\right| \leq C(1+t+r)^{-2}(1+|t-r|)^{-1} .
$$

Since $t^{-1}-r^{-1}=(r-t)(r t)^{-1}$, we obtain from (6.7) and (6.35) that

$$
\left|\left(\frac{\varepsilon}{t}-\frac{\varepsilon}{r}\right) \mathbf{A}[W](r-t, \omega) \partial_{-}(r v(t, r \omega))\right| \leq C \varepsilon^{2}(1+t+r)^{\lambda-2}(1+|t-r|)^{-\rho} .
$$

Note that we can replace $t^{-1}$ by $(1+t+r)^{-1}$ to derive of (6.39) because we have $A[W](r-t, \omega)=0$ for $r \geq t+R$. We define

$$
\begin{aligned}
G^{\#}(t, r, \omega) & =\left(G_{1}^{\#}(t, r, \omega), \ldots, G_{N^{\prime}}^{\#}(t, r, \omega)\right)^{\mathrm{T}} \\
& :=r\left(G_{j}(\omega, u(t, r \omega), \partial u(t, r \omega))\right)_{1 \leq j \leq N^{\prime}}^{\mathrm{T}}-\frac{\varepsilon}{r} \mathbf{A}[W](r-t, \omega) \partial_{-}(r v(t, r \omega)),
\end{aligned}
$$


where each $G_{j}$ is given by (2.12). By (2.22), (2.27), (3.10), and (6.7), we get

$$
\begin{aligned}
\mid r w_{l}( & t, r \omega)\left(\partial_{a} v_{k}\right)(t, r \omega)-\left(-\frac{\varepsilon \omega_{a}}{2 r}\right) W_{l}(r-t, \omega) \partial_{-}\left(r v_{k}(t, r \omega)\right) \mid \\
\leq & \left|\left(r w_{l}(t, r \omega)-\varepsilon W_{l}(r-t, \omega)\right)\left(\partial_{a} v_{k}\right)(t, r \omega)\right| \\
& +\left|\frac{\varepsilon}{r} W_{l}(r-t, \omega)\left(r\left(\partial_{a} v_{k}\right)(t, r \omega)-\left(-\frac{\omega_{a}}{2}\right) \partial_{-}\left(r v_{k}(t, r \omega)\right)\right)\right| \\
\leq & C \varepsilon^{2}(1+t+r)^{3 \lambda-2}(1+|t-r|)^{-\rho}
\end{aligned}
$$

for $1 \leq k \leq N^{\prime}$ and $1 \leq l \leq N^{\prime \prime}$. Similarly, using (2.23) instead of (2.22), we obtain

$$
\begin{aligned}
& \left|r\left(\partial_{b} w_{l}\right)(t, r \omega)\left(\partial_{a} v_{k}\right)(t, r \omega)-\left(-\frac{\varepsilon \omega_{a} \omega_{b}}{2 r}\right)\left(\partial_{\sigma} W_{l}\right)(r-t, \omega) \partial_{-}\left(r v_{k}(t, r \omega)\right)\right| \\
& \leq C \varepsilon^{2}(1+t+r)^{3 \lambda-2}(1+|t-r|)^{-1-\rho} .
\end{aligned}
$$

Thus, recalling (2.12) and (2.20), we get

$$
\left|G^{\#}(t, r, \omega)\right| \leq C \varepsilon^{2}(1+t+r)^{3 \lambda-2}(1+|t-r|)^{-\rho},
$$

which, together with (6.14), (6.16), and (6.39), yields

$$
\left|H_{j}^{\#}(t, r, \omega)\right| \leq C \varepsilon^{2}(1+t+r)^{3 \lambda-2}, \quad 1 \leq j \leq N^{\prime} .
$$

Hence by (6.36) and (6.38), we get

$$
\left|\Psi_{j}(t, r, \omega)\right| \leq C \varepsilon(1+t+r)^{3 \lambda+C \varepsilon-2}, \quad 1 \leq j \leq N^{\prime} .
$$

Now, we define $V_{j,-}=V_{j,-}(\sigma, \omega)$ by

$$
\varepsilon V_{j,-}(\sigma, \omega)=v_{j,-}^{\dagger}\left(t_{1}(\sigma), r_{1}(\sigma), \omega\right)+\int_{t_{1}(\sigma)}^{\infty} \Psi_{j}(\tau, \tau+\sigma, \omega) d \tau
$$

for $1 \leq j \leq N^{\prime}$. Then, from Lemma 6.1, (6.34), and (6.41), we obtain

$$
\left|v_{j,-}^{\dagger}(t, t+\sigma, \omega)-\varepsilon V_{j,-}(\sigma, \omega)\right| \leq C \varepsilon(1+2 t+\sigma)^{3 \lambda+C \varepsilon-1}
$$

for $1 \leq j \leq N^{\prime}, t \geq t_{0}(\sigma)$, and $(\sigma, \omega) \in \mathbb{R} \times S^{2}$. From (5.2) and (6.42) we get

$$
V_{j,-}(\sigma, \omega)=0, \quad \sigma \geq R, \omega \in S^{2} .
$$

Hence

$$
V_{j}(\sigma, \omega)=\frac{1}{2} \int_{\sigma}^{\infty} V_{j,-}(\tau, \omega) d \tau, \quad 1 \leq j \leq N^{\prime}
$$

is well-defined. We put $V=\left(V_{1}, \ldots, V_{N^{\prime}}\right)^{\mathrm{T}}$. Since $-2 \partial_{\sigma} V_{j}=V_{j,-},(\underline{6.36})$ and (6.43) lead to

$$
\begin{aligned}
& \left|\partial_{-}(r v(t, r \omega))-(-2 \varepsilon) e^{\boldsymbol{\Theta}_{\varepsilon}(t, r-t, \omega)}\left(\partial_{\sigma} V\right)(r-t, \omega)\right| \\
& \quad \leq\left\|e^{\boldsymbol{\Theta}_{\varepsilon}(t, r-t, \omega)}\right\|\left|e^{-\boldsymbol{\Theta}_{\varepsilon}(t, r-t, \omega)} \partial_{-}(r v(t, r \omega))-(-2 \varepsilon)\left(\partial_{\sigma} V\right)(r-t, \omega)\right| \\
& \quad \leq C \varepsilon(1+t+r)^{3 \lambda+C \varepsilon-1} .
\end{aligned}
$$

Finally, observing that (3.10) and (6.7) yield

$$
\left|r\left(\partial_{a} v\right)(t, r \omega)-\left(-\frac{\omega_{a}}{2}\right) \partial_{-}(r v(t, r \omega))\right| \leq C \varepsilon(1+t+r)^{\lambda-1},
$$

we obtain (2.21) from (6.46). 
Now it remains to prove (2.24). We set $v^{0, *}=\left(v_{j}^{0, *}\right)_{1 \leq j \leq N^{\prime}}^{\mathrm{T}}$, where $v_{j}^{0, *}(t, r, \omega)=$ $r v_{j}^{0}(t, r \omega)$. From (5.4) and (6.10) we find that

$$
\left|\left(\partial_{-} v_{j}^{0, *}\right)(t, t+\sigma, \omega)-(-2)\left(\partial_{\sigma} V_{j}^{0}\right)(\sigma, \omega)\right| \leq C(1+2 t+\sigma)^{-1}(1+|\sigma|)^{-1}
$$

for $t \geq t_{0}(\sigma)$ and $(\sigma, \omega) \in \mathbb{R} \times S^{2}$. If we put

$$
\begin{aligned}
V_{j,-}^{0}(\sigma, \omega):= & \left(\partial_{-} v_{j}^{0, *}\right)\left(t_{1}(\sigma), r_{1}(\sigma), \omega\right) \\
& +\left.\int_{t_{1}(\sigma)}^{\infty}\left(r^{-1}\left(\Delta_{\omega} v_{j}^{0}\right)(\tau, r \omega)\right)\right|_{r=\tau+\sigma} d \tau,
\end{aligned}
$$

then it follows from Lemma 6.1, (6.11) with $\psi=v_{j}^{0}$, and (6.38) that

$$
\left|\left(\partial_{-} v_{j}^{0, *}\right)(t, t+\sigma, \omega)-V_{j,-}^{0}(\sigma, \omega)\right| \leq C(1+2 t+\sigma)^{-1}(1+|\sigma|)^{-1}
$$

for $t \geq t_{0}(\sigma)$ and $(\sigma, \omega) \in \mathbb{R} \times S^{2}$. Now from (6.47) and (6.49) we get

$$
\left|-2\left(\partial_{\sigma} V_{j}^{0}\right)(\sigma, \omega)-V_{j,-}^{0}(\sigma, \omega)\right| \leq C \lim _{t \rightarrow \infty}(1+2 t+\sigma)^{-1}(1+|\sigma|)^{-1}=0,
$$

which shows

$$
-2\left(\partial_{\sigma} V_{j}^{0}\right)(\sigma, \omega)=V_{j,-}^{0}(\sigma, \omega), \quad(\sigma, \omega) \in \mathbb{R} \times S^{2} .
$$

We put $\widetilde{v}_{j}^{*}(t, r, \omega)=r \widetilde{v}_{j}(t, r \omega)$, and $\widetilde{v}^{*}=\left(\widetilde{v}_{j}^{*}\right)_{1 \leq j \leq N^{\prime}}^{\mathrm{T}}$. By (6.42), (6.45), (6.48), and (6.50), we obtain

$$
\begin{aligned}
& 2\left(\left(\partial_{\sigma} V\right)(\sigma, \omega)-\left(\partial_{\sigma} V^{0}\right)(\sigma, \omega)\right)=-\left(V_{j,-}(\sigma, \omega)-V_{j,-}^{0}(\sigma, \omega)\right)_{1 \leq j \leq N^{\prime}}^{\mathrm{T}} \\
& =-\left(e^{-\boldsymbol{\Theta}_{\varepsilon}\left(t_{1}(\sigma), \sigma, \omega\right)}-\mathbf{I}\right)\left(\partial_{-} v^{0, *}\right)\left(t_{1}(\sigma), r_{1}(\sigma), \omega\right) \\
& \quad-\varepsilon^{-1} e^{-\boldsymbol{\Theta}_{\varepsilon}\left(t_{1}(\sigma), \sigma, \omega\right)}\left(\partial_{-} \widetilde{v}^{*}\right)\left(t_{1}(\sigma), r_{1}(\sigma), \omega\right) \\
& \quad-\varepsilon^{-1} \int_{t_{1}(\sigma)}^{\infty} e^{-\boldsymbol{\Theta}_{\varepsilon}(\tau, \sigma, \omega)} H^{\#}(\tau, \tau+\sigma, \omega) d \tau \\
& \quad-\left.\int_{t_{1}(\sigma)}^{\infty}\left(r^{-1}\left(e^{-\boldsymbol{\Theta}_{\varepsilon}(\tau, \sigma, \omega)}-\mathbf{I}\right)\left(\Delta_{\omega} v^{0}\right)(\tau, r \omega)\right)\right|_{r=\tau+\sigma} d \tau .
\end{aligned}
$$

Now using (5.4), (6.1), (6.7), (6.36), (6.37), (6.38), and (6.40), we get

$$
\begin{aligned}
\left|\partial_{\sigma} V(\sigma, \omega)-\partial_{\sigma} V^{0}(\sigma, \omega)\right| \leq & C \varepsilon\left(1+t_{1}(\sigma)+r_{1}(\sigma)\right)^{\lambda+C \varepsilon}(1+|\sigma|)^{-1} \\
& +C \varepsilon \int_{t_{1}(\sigma)}^{\infty}(1+2 \tau+\sigma)^{3 \lambda+C \varepsilon-2} d \tau \\
\leq & C \varepsilon(1+|\sigma|)^{3 \lambda+C \varepsilon-1}
\end{aligned}
$$

which leads to (2.24). This completes the proof.

\section{Asymptotic Behavior in the Energy Sense}

The purpose of this section is to prove Corollary 2.8. We also revisit three examples stated in Section 2.4, and we will prove (2.35), (2.39), and (2.42).

To begin with, we observe that (2.21), (2.22), and (2.23) in Theorem 2.6 are actually valid for any $(t, r) \in[2, \infty) \times(0, \infty)$ and $\omega \in S^{2}$. Indeed what is left to 
prove is their validity for $(t, r) \in \Lambda_{1}$ and $\omega \in S^{2}$, where $\Lambda_{1}=\{(t, r) \in[2, \infty) \times$ $(0, \infty) ; r<t / 2\}$. We obtain from (6.7) that

$$
\sup _{\omega \in S^{2}}\left|r \partial_{a} v(t, r \omega)\right| \leq C \varepsilon\langle t+r\rangle^{\lambda-1}
$$

for $(t, r) \in \Lambda_{1}$, because we have $\langle t+r\rangle \leq C\langle t-r\rangle$ for $(t, r) \in \Lambda_{1}$. Similarly (2.26) and (6.36) imply

$$
\sup _{\omega \in S^{2}}\left|\varepsilon e^{(\varepsilon \log t) \mathbf{A}[W](r-t, \omega)}\left(\partial_{\sigma} V\right)(r-t, \omega)\right| \leq C \varepsilon\langle t+r\rangle^{3 \lambda+C \varepsilon-1}
$$

for $(t, r) \in \Lambda_{1}$. Equations (7.1) and (7.2) show that (2.21) holds also for $(t, r) \in \Lambda_{1}$ and $\omega \in S^{2}$. In the same fashion, it follows from (6.8) and (2.27) that

$$
\begin{aligned}
\sup _{\omega \in S^{2}} \sum_{l=0}^{1}\langle t-r\rangle^{l}( & \left.\sum_{|\alpha|=l}\left|r\left(\partial^{\alpha} w\right)(t, r \omega)\right|+\left|\varepsilon\left(\partial_{\sigma}^{l} W\right)(r-t, \omega)\right|\right) \\
& \leq C \varepsilon\langle t+r\rangle^{-\rho} \leq C \varepsilon\langle t+r\rangle^{-1}\langle t-r\rangle^{1-\rho}, \quad(t, r) \in \Lambda_{1},
\end{aligned}
$$

which shows that (2.22) and (2.23) are true also for $(t, r) \in \Lambda_{1}$ and $\omega \in S^{2}$.

The following is an immediate consequence of this observation.

Corollary 7.1. Assume that the assumptions of Theorem 2.6 are fulfilled, and $(f, g) \in D$. Let $v, w, V, W$, and $\lambda$ be as in Theorem 2.6. We define $\boldsymbol{\Theta}_{\varepsilon}^{+}$as in Corollary 2.8. We also set

$$
\begin{aligned}
V_{\sigma, *}^{a}(t, x) & :=\left.\left(\omega_{a} r^{-1}\left(\partial_{\sigma} V\right)(r-t, \omega)\right)\right|_{r=|x|, \omega=x /|x|}, \\
W_{\sigma, *}^{a}(t, x) & :=\left.\left(\omega_{a} r^{-1}\left(\partial_{\sigma} W\right)(r-t, \omega)\right)\right|_{r=|x|, \omega=x /|x|}
\end{aligned}
$$

for $0 \leq a \leq 3$. Then there exists a positive constant $C$ such that we have

$$
\begin{aligned}
& \left(\sum_{a=0}^{3}\left\|\partial_{a} v(t, \cdot)-\varepsilon e^{\Theta_{\varepsilon}^{+}(t, \cdot)} V_{\sigma, *}^{a}(t, \cdot)\right\|_{L^{2}\left(\mathbb{R}^{3}\right)}^{2}\right)^{1 / 2} \leq C \varepsilon(1+t)^{3 \lambda+C \varepsilon-(1 / 2)}, \\
& \left(\sum_{a=0}^{3}\left\|\partial_{a} w(t, \cdot)-\varepsilon W_{\sigma, *}^{a}(t, \cdot)\right\|_{L^{2}\left(\mathbb{R}^{3}\right)}^{2}\right)^{1 / 2} \leq C \varepsilon(1+t)^{2 \lambda-1}
\end{aligned}
$$

for $t \geq 2$ and sufficiently small $\varepsilon(>0)$.

Proof. Let $t \geq 2$, and let $\varepsilon(>0)$ be sufficiently small. Switching to the polar coordinates, we find from (2.21) for $(t, r) \in[2, \infty) \times(0, \infty)$ and $\omega \in S^{2}$ that the left-hand side of (7.6) is bounded by

$$
C \varepsilon\left(\int_{0}^{\infty}(1+t+r)^{6 \lambda+2 C \varepsilon-2} d r\right)^{1 / 2} \leq C \varepsilon(1+t)^{3 \lambda+C \varepsilon-(1 / 2)}
$$


which is the desired bound. Similarly (7.7) follows from (2.23) for $(t, r) \in[2, \infty) \times$ $(0, \infty)$ and $\omega \in S^{2}$, because we have

$$
C \varepsilon\left(\int_{0}^{\infty}(1+t+r)^{4 \lambda-2}(1+|t-r|)^{-2 \rho} d r\right)^{1 / 2} \leq C \varepsilon(1+t)^{2 \lambda-1}
$$

for $\rho>1 / 2$.

Corollary 2.8 follows from Lemma 3.10 and Corollary 7.1.

Proof of Corollary 2.8. Suppose that all the assumptions of Theorem 2.6 are fulfilled. Note that (2.26) and (2.27) imply $\partial_{\sigma} V_{j}, \partial_{\sigma} W_{k} \in L^{2}\left(\mathbb{R} \times S^{2}\right)$ for small $\varepsilon>0$. Remember the definition of the isometric isomorphism $\mathcal{T}: H_{0} \rightarrow L^{2}\left(\mathbb{R} \times S^{2}\right)$ given in Section 3.3. We set $\left(f_{j}^{+}, g_{j}^{+}\right)=\mathcal{T}^{-1}\left[\partial_{\sigma} V_{j}\right]\left(\in H_{0}\right)$ for $1 \leq j \leq N^{\prime}$, and

$$
v^{+}=\left(\varepsilon \mathcal{U}_{0}\left[f_{1}^{+}, g_{1}^{+}\right], \ldots, \varepsilon \mathcal{U}_{0}\left[f_{N^{\prime}}^{+}, g_{N^{\prime}}^{+}\right]\right)^{\mathrm{T}}
$$

Let $V_{\sigma, *}^{a}$ be as in Corollary 7.1. Then, since we have

$$
\varepsilon V_{\sigma, *}^{a}(t, r \omega)=\left(\varepsilon \omega_{a} r^{-1} \mathcal{T}\left[f_{j}^{+}, g_{j}^{+}\right](r-t, \omega)\right)_{1 \leq j \leq N^{\prime}}^{\mathrm{T}},
$$

Lemma 3.10 implies

$$
\lim _{t \rightarrow \infty}\left(\sum_{a=0}^{3}\left\|\partial_{a} v^{+}(t, \cdot)-\varepsilon V_{\sigma, *}^{a}(t, \cdot)\right\|_{L^{2}\left(\mathbb{R}^{3}\right)}^{2}\right)^{1 / 2}=0 .
$$

Now (2.28) follows from (7.6) in Corollary 7.1 with the help of (6.36).

By setting $\left(f_{N^{\prime}+k}^{+}, g_{N^{\prime}+k}^{+}\right)=\mathcal{T}^{-1}\left[\partial_{\sigma} W_{k}\right]$ for $1 \leq k \leq N^{\prime \prime}$, and using (17.7), we can show (2.29) similarly.

Another consequence of Lemma 3.10 and Corollary 7.1 is the following.

Lemma 7.2. Suppose that all the assumptions of Theorem 2.6 are fulfilled. Let $v$, $V$, and $W$ be as in Theorem 2.6. Assume that $\varepsilon$ is positive and small enough.

If $v$ is asymptotically free in the energy sense, then there exists a function $T^{+}=$ $T^{+}(\sigma, \omega) \in L^{2}\left(\mathbb{R} \times S^{2} ; \mathbb{R}^{N^{\prime}}\right)$ such that we have

$$
\lim _{t \rightarrow \infty} e^{(\varepsilon \log t) \mathbf{A}[W]} \partial_{\sigma} V=T^{+} \quad \text { in } L^{2}\left(\mathbb{R} \times S^{2} ; \mathbb{R}^{N^{\prime}}\right) .
$$

Proof. Choose $\left(f_{j}^{+}, g_{j}^{+}\right) \in \dot{H}^{1}\left(\mathbb{R}^{3}\right) \times L^{2}\left(\mathbb{R}^{3}\right)$ for $1 \leq j \leq N^{\prime}$ satisfying

$$
\lim _{t \rightarrow \infty}\left\|v_{j}(t, \cdot)-\mathcal{U}_{0}\left[\varepsilon f_{j}^{+}, \varepsilon g_{j}^{+}\right](t, \cdot)\right\|_{E}=0
$$

We set $T_{j}^{+}:=\mathcal{T}\left[f_{j}^{+}, g_{j}^{+}\right]$for $1 \leq j \leq N^{\prime}$, and $T^{+}:=\left(T_{1}^{+}, \ldots, T_{N^{\prime}}^{+}\right)^{\mathrm{T}}$. If we put

$$
T_{*}^{+, a}(t, x):=\left.\left(\omega_{a} r^{-1} T^{+}(r-t, \omega)\right)\right|_{r=|x|, \omega=x /|x|},
$$

then (7.8) and Lemma 3.10 imply

$$
\lim _{t \rightarrow \infty} \sum_{a=0}^{3}\left\|\partial_{a} v(t, \cdot)-\varepsilon T_{*}^{+, a}(t, \cdot)\right\|_{L^{2}\left(\mathbb{R}^{3}\right)}^{2}=0 .
$$


Let $V_{\sigma, *}^{a}$ and $\Theta_{\varepsilon}^{+}$be as in Corollary 17.1, Then (7.6) and (7.9) yield

$$
\lim _{t \rightarrow \infty} \sum_{a=0}^{3}\left\|e^{\Theta_{\varepsilon}^{+}(t, \cdot)} V_{\sigma, *}^{a}(t, \cdot)-T_{*}^{+, a}(t, \cdot)\right\|_{L^{2}\left(\mathbb{R}^{3}\right)}^{2}=0
$$

which leads to

$$
\lim _{t \rightarrow \infty} \int_{\omega \in S^{2}}\left(\int_{-t}^{\infty}\left|e^{(\varepsilon \log t) \mathbf{A}[W](\sigma, \omega)}\left(\partial_{\sigma} V\right)(\sigma, \omega)-T^{+}(\sigma, \omega)\right|^{2} d \sigma\right) d S_{\omega}=0 .
$$

By (2.26) and (6.36) we get

$$
\left\|e^{(\varepsilon \log t) \mathbf{A}[W]}\left(\partial_{\sigma} V\right)\right\|_{L^{2}\left((-\infty,-t) \times S^{2}\right)} \leq C(1+t)^{3 \lambda+C \varepsilon-(1 / 2)} \rightarrow 0, \quad t \rightarrow \infty .
$$

Since $T^{+} \in L^{2}\left(\mathbb{R} \times S^{2}\right)$, it is easy to see that $\left\|T^{+}\right\|_{L^{2}\left((-\infty,-t) \times S^{2}\right)}$ converges to 0 as $t \rightarrow \infty$. Now we obtain the desired result immediately.

Next we will observe how freely we can choose the values of the radiation fields. Let $\psi \in C_{0}^{\infty}\left(\mathbb{R}^{3}\right)$ be radially symmetric, namely there exists a function $\psi^{\mathrm{R}}$ such that $\psi(x)=\psi^{\mathrm{R}}(|x|)$ for $x \in \mathbb{R}^{3}$. Then, the Radon transform of $\psi$ can be written as

$$
\mathcal{R}[\psi](\sigma, \omega)=\int_{y^{\prime} \in \mathbb{R}^{2}} \psi^{\mathrm{R}}\left(\sqrt{\left|y^{\prime}\right|^{2}+\sigma^{2}}\right) d y^{\prime}=2 \pi \int_{|\sigma|}^{\infty} s \psi^{\mathrm{R}}(s) d s .
$$

We also get

$$
\partial_{\sigma} \mathcal{R}[\psi](\sigma, \omega)=-2 \pi \sigma \psi^{\mathrm{R}}(|\sigma|)
$$

Lemma 7.3. Given $\sigma_{0} \neq 0, \alpha \in \mathbb{R}$, and $\beta \in \mathbb{R}$, there exists a pair of functions $(\varphi, \psi) \in C_{0}^{\infty}\left(\mathbb{R}^{3}\right) \times C_{0}^{\infty}\left(\mathbb{R}^{3}\right)$ such that we have

$$
\mathcal{F}_{0}[\varphi, \psi]\left(\sigma_{0}, \omega\right)=\alpha,\left(\partial_{\sigma} \mathcal{F}_{0}[\varphi, \psi]\right)\left(\sigma_{0}, \omega\right)=\beta
$$

for any $\omega \in S^{2}$.

Proof. We can choose a smooth and even function $\zeta=\zeta(s)$, vanishing in some neighborhood of $s=0$ and vanishing also for large $|s|$, such that $\zeta\left(\left|\sigma_{0}\right|\right)=2 \alpha$ and $\zeta^{\prime}\left(\left|\sigma_{0}\right|\right)=2\left(\operatorname{sgn} \sigma_{0}\right) \beta$, where $\zeta^{\prime}(s)=(d \zeta / d s)(s)$. For $x \in \mathbb{R}^{3}$, we define $\varphi(x)=0$ and $\psi(x)=-|x|^{-1} \zeta^{\prime}(|x|)$. Then we have $\psi \in C_{0}^{\infty}\left(\mathbb{R}^{3}\right)$, and we can easily obtain (7.12) from (7.10) and (7.11).

Let us recall the systems (1.23), (2.36), and (2.40) in Examples [2.9, 2.10, and 2.11. We are going to show (2.35), (2.39), and (2.42). For simplicity we only consider the initial condition of the form (1.2), instead of (2.8). More precisely we prove the following.

Proposition 7.4. We put $\widetilde{\mathcal{X}}_{N}=C_{0}^{\infty}\left(\mathbb{R}^{3} ; \mathbb{R}^{N}\right) \times C_{0}^{\infty}\left(\mathbb{R}^{3} ; \mathbb{R}^{N}\right)$.

(1) There exist $(f, g) \in \widetilde{\mathcal{X}}_{2}$ and three positive constants $\varepsilon_{1}, C_{1}$, and $C_{2}$ such that

$$
C_{1} \varepsilon(1+t)^{C_{1} \varepsilon} \leq\|u(t)\|_{E} \leq C_{2} \varepsilon(1+t)^{C_{2} \varepsilon}, \quad t \geq 0,0<\varepsilon \leq \varepsilon_{1},
$$

where $u=\left(u_{1}, u_{2}\right)^{\mathrm{T}}$ is the global solution to (1.23) with (1.2). 
(2) There exist $(f, g) \in \widetilde{\mathcal{X}}_{3}$ and three positive constants $\varepsilon_{1}, C_{1}$, and $C_{2}$ such that

$$
C_{1}\left(\varepsilon+\varepsilon^{2} \log (1+t)\right) \leq\|u(t)\|_{E} \leq C_{2}\left(\varepsilon+\varepsilon^{2} \log (1+t)\right), \quad t \geq 0,0<\varepsilon \leq \varepsilon_{1},
$$

where $u=\left(v^{\mathrm{T}}, w\right)^{\mathrm{T}}=\left(\left(v_{1}, v_{2}\right), w\right)^{\mathrm{T}}$ is the global solution to (2.36) with (1.2).

(3) For any $(f, g) \in \widetilde{\mathcal{X}}_{3}$ with $(f, g) \not \equiv(0,0)$, there exist three positive constants $C_{1}$, $C_{2}$, and $\varepsilon_{1}$ such that

$$
C_{1} \varepsilon \leq\|u(t)\|_{E} \leq C_{2} \varepsilon, \quad t \geq 0,0<\varepsilon \leq \varepsilon_{1},
$$

where $u=\left(v^{\mathrm{T}}, w\right)^{\mathrm{T}}=\left(\left(v_{1}, v_{2}\right), w\right)^{\mathrm{T}}$ is the global solution to (2.40) with (1.2). However there exists some $(f, g) \in \widetilde{\mathcal{X}}_{3}$ such that $u$ is not asymptotically free in the energy sense for $0<\varepsilon \leq \varepsilon_{1}$.

Proof. In what follows, $V$ and $W$ are the modified and the standard asymptotic profiles for the system under consideration in each assertion. $V_{\sigma, *}^{a}, W_{\sigma, *}^{a}$ and $\boldsymbol{\Theta}_{\varepsilon}^{+}$ are defined as in Corollary 7.1 .

First we prove (1). We put $w=\partial_{1} u_{2}-\partial_{2} u_{1}$. Then, as we have shown, $\left(u_{1}, u_{2}, w\right)^{\mathrm{T}}$ satisfies (1.24) with (2.30) and (2.31). By (2.18), we see that for any $(f, g) \not \equiv(0,0)$ and $T>0$, there exist two positive constants $C_{T}$ and $\widetilde{\varepsilon}_{T}$, depending on $f, g$, and $T$, such that

$$
C_{T}^{-1} \varepsilon(1+T) \leq\|u(t)\|_{E} \leq C_{T} \varepsilon, \quad 0 \leq t \leq T, 0<\varepsilon \leq \widetilde{\varepsilon}_{T}
$$

We are going to prove the following: If we choose appropriate $(f, g) \in \widetilde{\mathcal{X}}_{2}$, then there exist three positive constants $C_{1}^{\prime}, C_{2}^{\prime}$, and $\varepsilon_{2}$ such that

$$
C_{1}^{\prime}(1+t)^{C_{1}^{\prime} \varepsilon} \leq\left(\sum_{a=0}^{3}\left(\left\|e^{\Theta_{\varepsilon}^{+}(t)} V_{\sigma, *}^{a}(t)\right\|_{L^{2}}^{2}+\left\|W_{\sigma, *}^{a}(t)\right\|_{L^{2}}^{2}\right)\right)^{1 / 2} \leq C_{2}^{\prime}(1+t)^{C_{2}^{\prime} \varepsilon}
$$

for $t \geq 2$ and $0<\varepsilon \leq \varepsilon_{2}$. Once (7.17) is established, we obtain the desired result from (7.6), (17.7), and (7.16). Indeed, if we choose sufficiently large $T(>0)$, then (7.6), (17.7), and (7.17) yield the desired estimate for $t \geq T$, while (7.16) implies the desired estimate for $0 \leq t \leq T$, provided that $\varepsilon$ is sufficiently small.

We start the proof of (7.17). We take

$$
\mathcal{O}=\left\{\omega=\left(\omega_{1}, \omega_{2}, \omega_{3}\right) \in S^{2} ; \omega_{1} \leq-1 / 4, \omega_{2} \leq-1 / 4\right\}
$$

Remembering (2.32), we get

$$
\sum_{a=0}^{3}\left\|e^{\Theta_{\varepsilon}^{+}(t)} V_{\sigma, *}^{a}\right\|_{L^{2}}^{2} \geq 2 \int_{\omega \in \mathcal{O}} \int_{-t}^{\infty} t^{-\varepsilon \omega_{1} W(\sigma, \omega)}\left|\partial_{\sigma} V_{1}(\sigma, \omega)\right|^{2} d \sigma d S_{\omega}
$$

for $t \geq 2$. Fix some $\sigma_{0}>0$. From Lemma 7.3 we can find $\left(f_{j}, g_{j}\right) \in \widetilde{\mathcal{X}}_{2}(j=1,2)$ such that

$$
\left(\partial_{\sigma} \mathcal{F}_{0}\left[f_{1}, g_{1}\right]\right)\left(\sigma_{0}, \omega\right)=-\left(\partial_{\sigma} \mathcal{F}_{0}\left[f_{2}, g_{2}\right]\right)\left(\sigma_{0}, \omega\right)=8, \quad \omega \in S^{2} .
$$


From the continuity of the radiation field, we can choose some $\delta>0$ such that

$$
\left(\partial_{\sigma} \mathcal{F}_{0}\left[f_{1}, g_{1}\right]\right)(\sigma, \omega),-\left(\partial_{\sigma} \mathcal{F}_{0}\left[f_{2}, g_{2}\right]\right)(\sigma, \omega) \geq 4, \quad\left|\sigma-\sigma_{0}\right|<\delta, \omega \in S^{2} .
$$

Then (2.31) leads to

$$
\mathcal{F}_{0}\left[f_{3}, g_{3}\right](\sigma, \omega)=\omega_{1}\left(\partial_{\sigma} \mathcal{F}_{0}\left[f_{2}, g_{2}\right]\right)(\sigma, \omega)-\omega_{2}\left(\partial_{\sigma} \mathcal{F}_{0}\left[f_{1}, g_{1}\right]\right)(\sigma, \omega) \geq 2
$$

for $\left|\sigma-\sigma_{0}\right|<\delta$ and $\omega \in \mathcal{O}$. Now, with this choice of $(f, g)$, (2.24) and (2.25) imply

$$
\partial_{\sigma} V_{1}(\sigma, \omega) \geq 2, W(\sigma, \omega) \geq 1, \quad\left|\sigma-\sigma_{0}\right|<\delta, \omega \in \mathcal{O}
$$

for sufficiently small $\varepsilon$. Since we may assume $\sigma_{0}-\delta>0>-t$, by (7.18) and (7.20) we obtain

$$
\sum_{a=0}^{3}\left\|e^{\Theta_{\varepsilon}^{+}(t)} V_{\sigma, *}^{a}(t)\right\|_{L^{2}}^{2} \geq C(1+t)^{\varepsilon / 4}
$$

for $t \geq 2$, and the first half of (7.17) is proved.

By (2.26) and (2.27), we have $\left\|\partial_{\sigma} V\right\|_{L_{\sigma, \omega}^{2}}+\left\|\partial_{\sigma} W\right\|_{L_{\sigma, \omega}^{2}} \leq C$ for small $\varepsilon>0$, where $L_{\sigma, \omega}^{2}$ denotes $L^{2}\left(\mathbb{R} \times S^{2}\right)$. We also have $t^{-\varepsilon \omega_{1} W / 2}+\left|\omega_{1}^{-1}\left(t^{-\varepsilon \omega_{1} W / 2}-1\right)\right| \leq C(1+t)^{C \varepsilon}$ for $t \geq 2$. Hence we get

$$
\begin{gathered}
\left(\sum_{a=0}^{3}\left\|e^{\Theta_{\varepsilon}^{+}(t)} V_{\sigma, *}^{a}(t)\right\|_{L^{2}}^{2}\right)^{1 / 2} \leq C(1+t)^{C \varepsilon}\left\|\partial_{\sigma} V\right\|_{L_{\sigma, \omega}^{2}} \leq C(1+t)^{C \varepsilon}, \\
\left(\sum_{a=0}^{3}\left\|W_{\sigma, *}^{a}(t)\right\|_{L^{2}}^{2}\right)^{1 / 2} \leq C\left\|\partial_{\sigma} W\right\|_{L_{\sigma, \omega}^{2}} \leq C,
\end{gathered}
$$

which imply the last half of (17.17).

Next we prove (2). Since (7.22) is valid also for this case, our task is to estimate $\sum_{a=0}^{3}\left\|e^{\boldsymbol{\Theta}_{\varepsilon}^{+}(t)} V_{\sigma, *}^{a}(t)\right\|_{L^{2}}^{2}$. Fix some $\sigma_{0}>0$. Similarly to (7.19), we can take some $(f, g) \in \widetilde{\mathcal{X}}_{3}$ and some $\delta>0$ such that

$$
\left(\partial_{\sigma} \mathcal{F}_{0}\left[f_{j}, g_{j}\right]\right)(\sigma, \omega) \geq 2, \quad\left|\sigma-\sigma_{0}\right|<\delta, \omega \in S^{2}, 1 \leq j \leq 3 .
$$

From (2.24) and (2.25), we get

$$
\partial_{\sigma} V_{1}(\sigma, \omega) \geq 1, \partial_{\sigma} V_{2}(\sigma, \omega) \geq 1, \partial_{\sigma} W(\sigma, \omega) \geq 1
$$

for $\left|\sigma-\sigma_{0}\right|<\delta$ and $\omega \in S^{2}$, provided that $\varepsilon$ is small enough. Since we may assume $\sigma_{0}-\delta>0$, from (2.37) we get

$$
\sum_{a=0}^{3}\left\|e^{\Theta_{\varepsilon}^{+}(t)} V_{\sigma, *}^{a}\right\|_{L^{2}}^{2} \geq 8 \pi \int_{\sigma_{0}-\delta}^{\sigma_{0}+\delta}|1+\varepsilon \log t|^{2} d \sigma \geq C(1+\varepsilon \log (1+t))^{2}
$$

for $t \geq 2$ and small $\varepsilon$. It follows from (2.26), (2.27), (2.37), and (2.38) that

$$
\begin{aligned}
\sum_{a=0}^{3}\left\|e^{\Theta_{\varepsilon}^{+}(t)} V_{\sigma, *}^{a}\right\|_{L^{2}}^{2} & \leq C\left(\left\|\partial_{\sigma} V\right\|_{L_{\sigma, \omega}^{2}}+(\varepsilon \log t)\left\|\partial_{\sigma} W\right\|_{L^{\infty}\left(\mathbb{R} \times S^{2}\right)}\left\|\partial_{\sigma} V_{2}\right\|_{L_{\sigma, \omega}^{2}}\right)^{2} \\
& \leq C(1+\varepsilon \log (1+t))^{2} .
\end{aligned}
$$


Now, using Corollary 7.1, we can easily reach at the desired result as before.

Finally we prove (3). From (2.40) we obtain

$$
\frac{d}{d t}\left(\|u(t)\|_{E}^{2}\right)=\sum_{j=1}^{3} \int_{\mathbb{R}^{3}} F_{j}^{0}(\partial u) \partial_{t} u_{j} d x .
$$

It follows from Lemma 3.3 (with $s=0$ ), (2.15), and (2.16) that

$$
\begin{aligned}
\sum_{j=1}^{3} \int_{\mathbb{R}^{3}}\left|F_{j}^{0}(\partial u) \partial_{t} u_{j}\right| d x & \leq C\left(\sup _{x \in \mathbb{R}^{3}}\langle t+|x|\rangle^{-1}|u(t, x)|_{1}\right)\|\partial u(t)\|_{L^{2}}^{2} \\
& \leq C \varepsilon^{3}(1+t)^{3 \lambda-2}
\end{aligned}
$$

Hence we get

$$
\left|\|u(t)\|_{E}^{2}-\|u(0)\|_{E}^{2}\right| \leq C \varepsilon^{3} \int_{0}^{\infty}(1+\tau)^{3 \lambda-2} d \tau \leq C \varepsilon^{3},
$$

which leads to (2.42) for small $\varepsilon>0$, because we have

$$
2\|u(0)\|_{E}^{2}=\varepsilon^{2}\left(\left\|\nabla_{x} f\right\|_{L^{2}}^{2}+\|g\|_{L^{2}}^{2}\right) .
$$

Now we are going to prove that $u$ is not asymptotically free in the energy sense for some initial profile. We fix some $\sigma_{0}>0$. Then, similarly to the above, we can take $(f, g) \in \widetilde{\mathcal{X}}_{3}$ and $\delta>0$ such that

$$
\partial_{\sigma} V(\sigma, \omega) \neq 0,1 \leq \partial_{\sigma} W(\sigma, \omega) \leq 2, \quad(\sigma, \omega) \in \mathcal{I} \times S^{2}
$$

for sufficiently small $\varepsilon>0$, where $\mathcal{I}=\left(\sigma_{0}-\delta, \sigma_{0}+\delta\right)$. Suppose that $u$ is asymptotically free in the energy sense. From Lemma 7.2 we see that $e^{(\varepsilon \log t) \mathbf{A}[W]}\left(\partial_{\sigma} V\right)$ converges to some function in $L^{2}\left(\mathbb{R} \times S^{2}\right)$ as $t$ goes to $\infty$. Therefore we get

$$
\lim _{t \rightarrow \infty}\left\|\left(e^{(\varepsilon \log (e t)) \mathbf{A}[W]}-e^{(\varepsilon \log t) \mathbf{A}[W]}\right)\left(\partial_{\sigma} V\right)\right\|_{L^{2}\left(\mathbb{R} \times S^{2}\right)}=0 .
$$

On the other hand, we get

$$
\begin{aligned}
& \left\|\left(e^{(\varepsilon \log (e t)) \mathbf{A}[W]}-e^{(\varepsilon \log t) \mathbf{A}[W]}\right)\left(\partial_{\sigma} V\right)\right\|_{L^{2}\left(\mathbb{R} \times S^{2}\right)} \\
& =\left\|\left(e^{\varepsilon \mathbf{A}[W]}-\mathbf{I}\right)\left(\partial_{\sigma} V\right)\right\|_{L^{2}\left(\mathbb{R} \times S^{2}\right)}
\end{aligned}
$$

for $t \geq 2$, because we have $\left|e^{(\varepsilon \log t) \mathbf{A}[W](\sigma, \omega)} Y\right|=|Y|$ for any $Y \in \mathbb{R}^{2}$ by (2.41). By (7.24) and (7.25) we conclude that $\left(e^{\varepsilon \mathbf{A}[W](\sigma, \omega)}-\mathbf{I}\right)\left(\partial_{\sigma} V\right)(\sigma, \omega)=0$ for almost every $(\sigma, \omega) \in \mathbb{R} \times S^{2}$. This contradicts (7.23) if $\varepsilon(>0)$ is small enough, because $e^{\varepsilon \mathbf{A}[W](\sigma, \omega)}-\mathbf{I}$ is invertible when $0<\varepsilon\left(\partial_{\sigma} W\right)(\sigma, \omega)<2 \pi$. Hence $u$ is not asymptotically free in the energy sense for small $\varepsilon$. 


\section{Proof of Theorems 2.14 and 2.15}

8.1. Proof of Theorem 2.14, Before we proceed to the proof of Theorem 2.14, we recall some elementary facts from the linear algebra.

Let $m$ be a positive integer, and $\mathbf{I}$ be the $m \times m$ identity matrix. Suppose that $\mathbf{B}$ is an $m \times m$ complex matrix. For a complex number $\mu$ and a positive integer $n$, we define $\mathcal{K}_{\mu}^{n}(\mathbf{B})=\operatorname{ker}(\mathbf{B}-\mu \mathbf{I})^{n}=\left\{y \in \mathbb{C}^{m} ;(\mathbf{B}-\mu \mathbf{I})^{n} y=0\right\}$. We also define $\mathcal{K}_{\mu}(\mathbf{B})=\bigcup_{n=1}^{\infty} \mathcal{K}_{\mu}^{n}(\mathbf{B})$. It is known that for any $\mu \in \mathbb{C}$, there exists a positive integer $l(\leq m)$ such that $\mathcal{K}_{\mu}^{l}(\mathbf{B})=\mathcal{K}_{\mu}(\mathbf{B})$. Let $\mu_{1}, \ldots, \mu_{J}$ (with some $J \leq m$ ) be all of the distinct eigenvalues of $\mathbf{B}$. Then it is well known that we have

$$
\mathbb{C}^{m}=\bigoplus_{j=1}^{J} \mathcal{K}_{\mu_{j}}(\mathbf{B})
$$

For $y \in \mathcal{K}_{\mu_{j}}(\mathbf{B})$, we get

$$
e^{\tau \mathbf{B}} y=e^{\tau \mu_{j} \mathbf{I}} e^{\tau\left(\mathbf{B}-\mu_{j} \mathbf{I}\right)} y=e^{\mu_{j} \tau} \sum_{n=0}^{l_{j}-1} \frac{\tau^{n}}{n !}\left(\mathbf{B}-\mu_{j} \mathbf{I}\right)^{n} y,
$$

where $l_{j}$ is the smallest positive integer to satisfy $\mathcal{K}_{\mu_{j}}^{l_{j}}(\mathbf{B})=\mathcal{K}_{\mu_{j}}(\mathbf{B})$. We define

$$
\mathcal{Z}(\mathbf{B})=\mathcal{K}_{0}^{1}(\mathbf{B}) \oplus \bigoplus_{1 \leq j \leq J ; \operatorname{Re} \mu_{j}<0} \mathcal{K}_{\mu_{j}}(\mathbf{B}) .
$$

Note that $y \in \mathcal{Z}(\mathbf{B})$ implies

$$
\left(\mathbf{B} \prod_{1 \leq j \leq J ; \operatorname{Re} \mu_{j}<0}\left(\mathbf{B}-\mu_{j} \mathbf{I}\right)^{m}\right) y=0 .
$$

(8.1) and (8.2) lead to the following property:

Lemma 8.1. $e^{\tau \mathbf{B}} y$ converges to some vector in $\mathbb{C}^{m}$ as $\tau \rightarrow \infty$, if and only if

$$
y \in \mathcal{Z}(\mathbf{B}) \text {. }
$$

The following two lemmas play important roles in the proof of Theorem 2.14

Lemma 8.2. Let $\boldsymbol{\Phi}_{0}$ be an $m \times m$ matrix. If $\boldsymbol{\Phi}_{0}$ has an eigenvalue $\lambda$ whose real part is positive, then there exists a vector $z^{0}=z^{0}\left(\boldsymbol{\Phi}_{0}\right) \in \mathbb{C}^{m}$ such that the following holds: Suppose that $\boldsymbol{\Phi}=\boldsymbol{\Phi}(\varepsilon)$ be an $m \times m$ matrix-valued function of $\varepsilon \in[0,1]$, say; if $\boldsymbol{\Phi}=\boldsymbol{\Phi}(\varepsilon)$ is continuous at $\varepsilon=0$, and $\boldsymbol{\Phi}(0)=\boldsymbol{\Phi}_{0}$, then there exist two positive constants $\varepsilon_{1}$ and $\delta$ such that

$$
\mathcal{Z}(\boldsymbol{\Phi}(\varepsilon)) \cap B_{\delta}\left(z^{0}\right)=\emptyset
$$

for any $\varepsilon \in\left[0, \varepsilon_{1}\right]$, where $B_{\delta}\left(z^{0}\right)=\left\{y \in \mathbb{C}^{m} ;\left|y-z^{0}\right| \leq \delta\right\}$.

Furthermore, if $\boldsymbol{\Phi}_{0}$ is a real matrix, then we can choose $z^{0}$ in the above from $\mathbb{R}^{m}$. 
Proof. We denote the eigenvalues of $\boldsymbol{\Phi}(\varepsilon)$ by $\lambda_{1}(\varepsilon), \ldots, \lambda_{m}(\varepsilon)$, with each eigenvalue being counted up to its algebraic multiplicity. Without loss of generality, we may assume that each $\lambda_{j}(\varepsilon)$ is continuous at $\varepsilon=0$. Note that $\lambda_{1}(0), \ldots, \lambda_{m}(0)$ are the eigenvalues of $\boldsymbol{\Phi}_{0}$.

If all the real parts of the eigenvalues of $\boldsymbol{\Phi}_{0}$ are positive, then (8.4) is trivial, since $\operatorname{Re} \lambda_{j}(\varepsilon)>0$ for $1 \leq j \leq m$, provided that $\varepsilon$ is small.

Suppose that the real part of some eigenvalue of $\boldsymbol{\Phi}_{0}$ is non-positive. Then, without loss of generality, we may assume that $\operatorname{Re} \lambda_{j}(0)>0$ for $1 \leq j \leq m^{\prime}$ and $\operatorname{Re} \lambda_{j}(0) \leq 0$ for $m^{\prime}+1 \leq j \leq m$ with some positive integer $m^{\prime}(<m)$. We define

$$
\boldsymbol{\Psi}(\varepsilon)=\boldsymbol{\Phi}(\varepsilon)\left(\boldsymbol{\Phi}(\varepsilon)-\lambda_{m^{\prime}+1}(\varepsilon) \mathbf{I}\right)^{m} \cdots\left(\boldsymbol{\Phi}(\varepsilon)-\lambda_{m}(\varepsilon) \mathbf{I}\right)^{m}, \quad \varepsilon \geq 0 .
$$

We are going to show that there exists $z^{0} \in \mathbb{C}^{m}$, depending only on $\boldsymbol{\Phi}_{0}$, such that

$$
\Psi(0) z^{0} \neq 0 \text {. }
$$

Let $z^{1} \in \mathbb{C}^{m}$ be an eigenvector of $\boldsymbol{\Phi}_{0}$ associated with the eigenvalue $\lambda_{1}(0)(>0)$. Then we have

$$
\Psi(0) z^{1}=\lambda_{1}(0)\left(\lambda_{1}(0)-\lambda_{m^{\prime}+1}(0)\right)^{m} \cdots\left(\lambda_{1}(0)-\lambda_{m}(0)\right)^{m} z^{1} \neq 0,
$$

and we obtain (8.5) with $z^{0}=z^{1}$. If $\boldsymbol{\Phi}_{0}$ is a real matrix, then the same is true for $\boldsymbol{\Psi}(0)$ (observe that if $\lambda$ is an eigenvalue of $\boldsymbol{\Phi}_{0}$, so is its complex conjugate $\bar{\lambda}$ ), and we have either $\boldsymbol{\Psi}(0)\left(\operatorname{Re} z^{1}\right) \neq 0$ or $\boldsymbol{\Psi}(0)\left(\operatorname{Im} z^{1}\right) \neq 0$. Thus we can choose $z^{0} \in \mathbb{R}^{m}$ satisfying (8.5).

Suppose that $z^{0}$ satisfies (8.5). From the continuity of $\boldsymbol{\Psi}(\varepsilon)$ at $\varepsilon=0$, the mapping $(\varepsilon, y) \mapsto \Psi(\varepsilon) y$ is continuous at $(\varepsilon, y)=\left(0, z^{0}\right)$. Hence there exists $\varepsilon_{2}>0$ and $\delta>0$ such that $\Psi(\varepsilon) y \neq 0$ for any $y \in B_{\delta}\left(z^{0}\right)$ and $0 \leq \varepsilon \leq \varepsilon_{2}$. This leads to (8.4), because $y \in \mathcal{Z}(\boldsymbol{\Phi}(\varepsilon))$ implies $\boldsymbol{\Psi}(\varepsilon) y=0$ if $\varepsilon(>0)$ is sufficiently small (cf. (8.3); observe that $\operatorname{Re} \lambda_{j}(\varepsilon) \leq 0$ implies $m^{\prime}+1 \leq j \leq m$ for small $\varepsilon$ ).

Lemma 8.3. Let $\boldsymbol{\Phi}=\boldsymbol{\Phi}(\varepsilon)$ be an $m \times m$ real matrix-valued function, and $\varepsilon_{1}$ be a positive constant. Assume that $\boldsymbol{\Phi}=\boldsymbol{\Phi}(\varepsilon)$ is continuous at $\varepsilon=0$, and that $\boldsymbol{\Phi}(0) \neq \mathbf{O}$, where $\mathbf{O}$ denotes the zero matrix.

If all the real parts of the eigenvalues of $\boldsymbol{\Phi}(\varepsilon)$ are zero for $0 \leq \varepsilon \leq \varepsilon_{1}$, then for any $z \in \mathbb{R}^{m}$ satisfying $\boldsymbol{\Phi}(0) z \neq 0$, there exist two positive constant $\varepsilon_{2}\left(\leq \varepsilon_{1}\right)$ and $\delta$ such that $\mathcal{Z}(\boldsymbol{\Phi}(\varepsilon)) \cap B_{\delta}(z)=\emptyset$ holds for any $\varepsilon \in\left[0, \varepsilon_{2}\right]$.

Proof. Suppose $\boldsymbol{\Phi}(0) z \neq 0$. Then, from the continuity of the mapping $(\varepsilon, y) \mapsto$ $\boldsymbol{\Phi}(\varepsilon) y$ at $(\varepsilon, y)=(0, z)$, there exist two positive constant $\varepsilon_{2}\left(\leq \varepsilon_{1}\right)$ and $\delta$ such that $\Phi(\varepsilon) y \neq 0$ for $0 \leq \varepsilon \leq \varepsilon_{2}$ and $y \in B_{\delta}(z)$. This completes the proof because $\mathcal{Z}(\boldsymbol{\Phi}(\varepsilon))=\mathcal{K}_{0}^{1}(\boldsymbol{\Phi}(\varepsilon))$ from the assumption.

Now we are in a position to prove Theorem 2.14. (AFP) under the null condition follows immediately from Theorem 2.6 by regarding $w=u$ and neglecting $v$. Hence we only have to prove that (2) implies (1). 
Suppose that Condition 2.2 is satisfied with $D=\mathcal{X}_{N}$, and (AFP) holds. We define $\mathbf{B}(\omega, \xi, \eta)=\left(B_{j k}(\omega, \xi, \eta)\right)_{1 \leq j, k \leq N^{\prime}}$ by

$$
B_{j k}(\omega, \xi, \eta)=-\frac{1}{2} \sum_{a=0}^{3} \omega_{a} \sum_{l=1}^{N^{\prime \prime}}\left(c_{j k}^{a, l}(\omega) \xi_{l}+\sum_{b=0}^{3} d_{j k}^{a, l b}(\omega) \omega_{b} \eta_{l}\right)
$$

for $\omega=\left(\omega_{1}, \omega_{2}, \omega_{3}\right) \in S^{2}$, and $\xi=\left(\xi_{l}\right)_{1 \leq l \leq N^{\prime \prime}}^{\mathrm{T}}, \eta=\left(\eta_{l}\right)_{1 \leq l \leq N^{\prime \prime}}^{\mathrm{T}} \in \mathbb{R}^{N^{\prime \prime}}$, where $c_{j k}^{a, l}$ and $d_{j k}^{a, l b}$ are from (2.12). Observe that we have

$$
\mathbf{A}[\zeta](\sigma, \omega)=\mathbf{B}\left(\omega, \zeta(\sigma, \omega),\left(\partial_{\sigma} \zeta\right)(\sigma, \omega)\right),
$$

where $\mathbf{A}[\zeta](\sigma, \omega)$ is given by (2.19) $-(2.20)$.

Suppose that there exists some $\left(\omega^{\prime}, \xi^{\prime}, \eta^{\prime}\right) \in S^{2} \times \mathbb{R}^{N^{\prime \prime}} \times \mathbb{R}^{N^{\prime \prime}}$ such that $\mathbf{A}_{0}:=$ $\mathbf{B}\left(\omega^{\prime}, \xi^{\prime}, \eta^{\prime}\right)$ has an eigenvalue $\lambda$ with $\operatorname{Re} \lambda \neq 0$. We may assume $\operatorname{Re} \lambda>0$, because unless so, $-\lambda$ is an eigenvalue of $\mathbf{B}\left(\omega^{\prime},-\xi^{\prime},-\eta^{\prime}\right)$ with $\operatorname{Re}(-\lambda)>0$. We put $z^{\prime}=$ $z^{0}\left(\mathbf{A}_{0}\right)$, where $z^{0}\left(\mathbf{A}_{0}\right)$ is from Lemma 8.2 with $m=N^{\prime}$ and $\boldsymbol{\Phi}_{0}=\mathbf{A}_{0}$. Note that we may assume $z^{\prime} \in \mathbb{R}^{N^{\prime}}$. We fix some $\sigma^{\prime} \neq 0$. Writing $\xi^{\prime}=\left(\xi_{k}^{\prime}\right)_{1 \leq k \leq N^{\prime \prime}}^{\mathrm{T}}, \eta^{\prime}=$ $\left(\eta_{k}^{\prime}\right)_{1 \leq k \leq N^{\prime \prime}}^{\mathrm{T}}$, and $z^{\prime}=\left(z_{j}^{\prime}\right)_{1 \leq j \leq N^{\prime}}^{\mathrm{T}}$, by Lemma 7.3 we can find $(f, g) \in C_{0}^{\infty}\left(\mathbb{R}^{3} ; \mathbb{R}^{N}\right) \times$ $C_{0}^{\infty}\left(\mathbb{R}^{3} ; \mathbb{R}^{N}\right)$ such that we have

$$
\begin{aligned}
& \left(\partial_{\sigma} \mathcal{F}_{0}\left[f_{j}, g_{j}\right]\right)\left(\sigma^{\prime}, \omega\right)=z_{j}^{\prime}, \\
& \mathcal{F}_{0}\left[f_{N^{\prime}+k}, g_{N^{\prime}+k}\right]\left(\sigma^{\prime}, \omega\right)=\xi_{k}^{\prime}, \quad\left(\partial_{\sigma} \mathcal{F}_{0}\left[f_{N^{\prime}+k}, g_{N^{\prime}+k}\right]\right)\left(\sigma^{\prime}, \omega\right)=\eta_{k}^{\prime}
\end{aligned}
$$

for $1 \leq j \leq N^{\prime}, 1 \leq k \leq N^{\prime \prime}$, and $\omega \in S^{2}$. Let $V$ and $W$ be the modified and the standard asymptotic profiles corresponding to $(f, g)$ above. We write $V=V(\sigma, \omega ; \varepsilon)$ and $W=W(\sigma, \omega ; \varepsilon)$ to indicate the dependence on the parameter $\varepsilon$ explicitly. We put $\mathbf{A}_{\varepsilon}=\mathbf{A}[W(\cdot, \cdot ; \varepsilon)]\left(\sigma^{\prime}, \omega^{\prime}\right)$ for $\varepsilon>0$. Then, by (2.25), we get

$$
\mathbf{A}_{\varepsilon}=\mathbf{B}\left(\omega^{\prime}, W\left(\sigma^{\prime}, \omega^{\prime} ; \varepsilon\right),\left(\partial_{\sigma} W\right)\left(\sigma^{\prime}, \omega^{\prime} ; \varepsilon\right)\right) \rightarrow \mathbf{B}\left(\omega^{\prime}, \xi^{\prime}, \eta^{\prime}\right)=\mathbf{A}_{0}
$$

as $\varepsilon \rightarrow+0$. Hence, from Lemma 8.2 , there exist $\varepsilon_{1}>0$ and $\delta>0$ such that

$$
\mathcal{Z}\left(\mathbf{A}_{\varepsilon}\right) \cap B_{\delta}\left(z^{\prime}\right)=\emptyset
$$

holds for $\varepsilon \in\left[0, \varepsilon_{1}\right]$. Since (2.24) and (8.6) imply $\left(\partial_{\sigma} V\right)\left(\sigma^{\prime}, \omega^{\prime} ; \varepsilon\right) \in B_{\delta}\left(z^{\prime}\right)$ for sufficiently small $\varepsilon(>0)$, we obtain

$$
\left(\partial_{\sigma} V\right)\left(\sigma^{\prime}, \omega^{\prime} ; \varepsilon\right) \notin \mathcal{Z}\left(\mathbf{A}_{\varepsilon}\right)
$$

for sufficiently small $\varepsilon(>0)$. From (AFP) and (2.21), $\varepsilon e^{\tau \mathbf{A}_{\varepsilon}}\left(\partial_{\sigma} V\right)\left(\sigma^{\prime}, \omega^{\prime} ; \varepsilon\right)$ must converge to some vector in $\mathbb{R}^{N^{\prime}}$ as $\tau \rightarrow \infty$, provided that $\varepsilon(>0)$ is sufficiently small. However this never occurs because of (8.8) and Lemma 8.1. Hence we conclude that all the real parts of the eigenvalues of $\mathbf{B}(\omega, \xi, \eta)$ must vanish for any $(\omega, \xi, \eta) \in S^{2} \times \mathbb{R}^{N^{\prime \prime}} \times \mathbb{R}^{N^{\prime \prime}}$

We fix arbitrary $\left(\omega^{\prime}, \xi^{\prime}, \eta^{\prime}\right) \in S^{2} \times \mathbb{R}^{N^{\prime \prime}} \times \mathbb{R}^{N^{\prime \prime}}$, and put $\mathbf{A}_{0}=\mathbf{B}\left(\omega^{\prime}, \xi^{\prime}, \eta^{\prime}\right)$ as before. Suppose that $\mathbf{A}_{0} \neq \mathbf{O}$. Then we can find a vector $z^{\prime} \in \mathbb{R}^{N^{\prime}}$ such that $\mathbf{A}_{0} z^{\prime} \neq 0$. Now, for this new choice of $\omega^{\prime}, \xi^{\prime}, \eta^{\prime}$, and $z^{\prime}$, we choose $(f, g)$ satisfying 
(8.6) and (8.7). Then, following the similar lines to the above, but using Lemma 8.3 instead of Lemma 8.2, we reach at (8.8) again, which is a contradiction. Hence we conclude $\mathbf{A}_{0}=\mathbf{O}$. Because $\left(\omega^{\prime}, \xi^{\prime}, \eta^{\prime}\right)$ can be chosen arbitrarily, this means that

$$
\mathbf{B}(\omega, \xi, \eta)=\mathbf{O}, \quad(\omega, \xi, \eta) \in S^{2} \times \mathbb{R}^{N^{\prime}} \times \mathbb{R}^{N^{\prime}} .
$$

Therefore $\sum_{a=0}^{3} \omega_{a} c_{j k}^{a, l}(\omega)=\sum_{a, b=0}^{3} \omega_{a} \omega_{b} d_{j k}^{a, l b}(\omega)=0$ for any $\omega \in S^{2}$. Remembering that we have $\partial_{a}=Z_{a}-\omega_{a} \partial_{t}$, and looking at (2.12), we get

$$
\begin{aligned}
G_{j}(\omega, u, \partial u)= & \sum_{a=0}^{3} \sum_{k=1}^{N^{\prime}} \sum_{l=1}^{N^{\prime \prime}}\left(c_{j k}^{a, l}(\omega) w_{l}+\sum_{b=0}^{3} d_{j k}^{a, l b}(\omega) \partial_{b} w_{l}\right)\left(Z_{a} v_{k}\right) \\
& -\sum_{a=0}^{3} \sum_{k=1}^{N^{\prime}} \sum_{l=1}^{N^{\prime \prime}} \sum_{b=0}^{3} d_{j k}^{a, l b}(\omega)\left(Z_{b} w_{l}\right)\left(\omega_{a} \partial_{t} v_{k}\right)
\end{aligned}
$$

for a smooth solution $u=\left(v^{\mathrm{T}}, w^{\mathrm{T}}\right)^{\mathrm{T}}$ to (1.1)-(2.8) on $[0, T) \times \mathbb{R}^{3}$, and we obtain $\left|G_{j}(\omega, u, \partial u)\right| \leq C(|u|+|\partial u|)|Z u|$. Now, from (2.13), (2.9), and Lemma 3.7 we get

$$
|F(u(t, r \omega), \partial u(t, r \omega))| \leq C\langle t+r\rangle^{-1}\left(|u(t, r \omega)|_{1}+|\partial u(t, r \omega)|\right)|u(t, r \omega)|_{1}
$$

for any $\omega \in S^{2}$ and $(t, r) \in[0, T) \times[0, \infty)$ satisfying $r \geq t / 2 \geq 1$.

We fix $\sigma_{0} \neq 0$. Let $(X, Y) \in \mathbb{R}^{N} \times \mathbb{R}^{N}$ be given. By Lemma 7.3, we can choose $(f, g) \in C_{0}^{\infty}\left(\mathbb{R}^{3} ; \mathbb{R}^{N}\right) \times C_{0}^{\infty}\left(\mathbb{R}^{3} ; \mathbb{R}^{N}\right)$ such that

$$
\left(\mathcal{F}_{0}\left[f_{j}, g_{j}\right]\left(\sigma_{0}, \omega\right),\left(\partial_{\sigma} \mathcal{F}_{0}\left[f_{j}, g_{j}\right]\right)\left(\sigma_{0}, \omega\right)\right)=\left(X_{j}, Y_{j}\right), \quad \omega \in S^{2}, 1 \leq j \leq N .
$$

Let $u=\left(v^{\mathrm{T}}, w^{\mathrm{T}}\right)^{\mathrm{T}}$ be the solution to (1.1) $-(2.8)$ with this choice of $(f, g)$ from now on. We write $u=u(t, x ; \varepsilon)$ in order to indicate the dependence on $\varepsilon$ explicitly. Let $V$ and $W$ be the modified and the standard asymptotic profiles given by Theorem 2.6. Note that, from (8.9) and (2.21), we have

$$
\sum_{a=0}^{3}\left|r\left(\partial_{a} v\right)(t, r \omega)-\varepsilon \omega_{a}\left(\partial_{\sigma} V\right)(r-t, \omega)\right| \leq C \varepsilon\langle t+r\rangle^{3 \lambda+C \varepsilon-1}
$$

for $r \geq t / 2 \geq 1$ and $\omega \in S^{2}$. We put $U=\left(V^{\mathrm{T}}, W^{\mathrm{T}}\right)^{\mathrm{T}}$. From (2.11), (8.12), (2.22), and (2.23), we get

$$
\begin{gathered}
\left.\lim _{t \rightarrow \infty}\left\{\varepsilon^{-2} r^{2} F(u(t, r \omega ; \varepsilon), \partial u(t, r \omega ; \varepsilon))\right\}\right|_{r=t+\sigma} \\
=F^{\mathrm{red}}\left(\omega, U(\sigma, \omega ; \varepsilon), \partial_{\sigma} U(\sigma, \omega ; \varepsilon)\right)
\end{gathered}
$$

for any fixed $(\sigma, \omega) \in \mathbb{R} \times S^{2}$. On the other hand, using (6.7) and (6.8) to evaluate the right-hand side of (8.10), we obtain

$$
\left|\left\{\varepsilon^{-2} r^{2} F(u(t, r \omega ; \varepsilon), \partial u(t, r \omega ; \varepsilon))\right\}\right|_{r=t+\sigma} \mid \leq C\langle 2 t+\sigma\rangle^{2 \lambda-1} \rightarrow 0, \quad t \rightarrow \infty .
$$

It follows from (8.13) and (8.14) that

$$
F^{\mathrm{red}}\left(\omega, U\left(\sigma_{0}, \omega ; \varepsilon\right), \partial_{\sigma} U\left(\sigma_{0}, \omega ; \varepsilon\right)\right)=0, \quad \omega \in S^{2} .
$$


Taking the limit in (8.15) as $\varepsilon \rightarrow+0$, we obtain from (2.24), (2.25), and (8.11) that $F^{\text {red }}(\omega, X, Y)=0$ for $\omega \in S^{2}$ and $1 \leq j \leq N$ (note that $F^{\text {red }}$ does not depend on $X_{j}$ with $1 \leq j \leq N^{\prime}$ because of (2.11) $)$. Since $(X, Y) \in \mathbb{R}^{N} \times \mathbb{R}^{N}$ can be chosen arbitrarily, the null condition (1.3) is satisfied. This completes the proof.

8.2. Proof of Theorem 2.15. Before we start the proof of Theorem 2.15, we investigate $e^{\tau \mathbf{B}}$ for a real matrix $\mathbf{B}$ whose rank is at most one. Let $p=\left(p_{1}, \ldots, p_{N}\right)^{\mathrm{T}}$, $q=\left(q_{1}, \ldots, q_{N}\right)^{\mathrm{T}} \in \mathbb{R}^{N}$. We put

$$
\mathbf{B}=q p^{\mathrm{T}}=\left(\begin{array}{ccc}
p_{1} q_{1} & \cdots & p_{N} q_{1} \\
\vdots & \ddots & \vdots \\
p_{1} q_{N} & \cdots & p_{N} q_{N}
\end{array}\right)
$$

For any $y=\left(y_{1}, \ldots, y_{N}\right)^{\mathrm{T}} \in \mathbb{R}^{N}$, we have $\mathbf{B} y=q p^{\mathrm{T}} y=\langle p, y\rangle q$, where $\langle\cdot, \cdot\rangle$ denotes the inner product in $\mathbb{R}^{N}$. Hence, for a positive integer $k$, we have

$$
\mathbf{B}^{k} y=\langle p, y\rangle \mathbf{B}^{k-1} q=\langle p, y\rangle\langle p, q\rangle^{k-1} q, \quad y \in \mathbb{R}^{N},
$$

and we get

$$
e^{\tau \mathbf{B}} y= \begin{cases}y+\tau\langle p, y\rangle q, & \text { if }\langle p, q\rangle=0 \\ y+\langle p, q\rangle^{-1}\langle p, y\rangle\left(e^{\langle p, q\rangle \tau}-1\right) q, & \text { if }\langle p, q\rangle \neq 0\end{cases}
$$

for any $y \in \mathbb{R}^{N}$. We need the following lemma for the proof of Theorem 2.15,

Lemma 8.4. Let $\mathbf{C}$ be an $N \times N$ real matrix, and $b \in \mathbb{R}^{N}$. Suppose that there exist two vectors $y, y^{\prime} \in \mathbb{R}^{N}$ such that

$$
\langle\mathbf{C} y, y\rangle \neq 0, \quad\left\langle\mathbf{C} y^{\prime}, b\right\rangle \neq 0 .
$$

Then there exists $z \in \mathbb{R}^{N}$ such that we have

$$
\langle\mathbf{C} z, z\rangle \neq 0 \text {, and }\langle\mathbf{C} z, b\rangle>0 .
$$

Proof. It suffices to prove the existence of $z$ satisfying (8.17) with $\langle\mathbf{C} z, b\rangle>0$ being replaced by $\langle\mathbf{C} z, b\rangle \neq 0$, because either $z$ or $-z$ has the desired property, depending on the sign of $\langle\mathbf{C} z, b\rangle$. The case where we have either $\langle\mathbf{C} y, b\rangle \neq 0$ or $\left\langle\mathbf{C} y^{\prime}, y^{\prime}\right\rangle \neq 0$ is triviality. Hence we suppose $\langle\mathbf{C} y, b\rangle=\left\langle\mathbf{C} y^{\prime}, y^{\prime}\right\rangle=0$, and we put $z=y+\lambda y^{\prime}$ for some $\lambda \neq 0$. Then we have $\langle\mathbf{C} z, b\rangle=\lambda\left\langle\mathbf{C} y^{\prime}, b\right\rangle \neq 0$. Since $\langle\mathbf{C} z, z\rangle=\langle\mathbf{C} y, y\rangle+\lambda\left(\left\langle\mathbf{C} y, y^{\prime}\right\rangle+\left\langle\mathbf{C} y^{\prime}, y\right\rangle\right)$, we find that $\langle\mathbf{C} z, z\rangle \neq 0$ if $|\lambda|$ is sufficiently small. This completes the proof.

Now we start the proof of Theorem 2.15, (AFP) and (AFE) under the null condition follow from Theorem 2.6 and Corollary 2.8, respectively. Hence it suffices to prove that if (1) does not hold, then neither (2) nor (3) holds.

Suppose that Condition 1.1 is fulfilled, but the null condition is not satisfied. As we have seen in Section 4, we can apply Theorem 2.5 to the extended system (4.6), 
and we can obtain a global solution $u=\left(u_{j}\right)_{1 \leq j \leq N}$ to the original Cauchy problem (1.1)-(2.8) with $F=F(\partial u)$ for sufficiently small $\varepsilon(>0)$.

Since the null condition is not satisfied, there exist some $\omega^{\prime} \in S^{2}$ and $Y^{\prime} \in \mathbb{R}^{N}$ such that $F^{\mathrm{red}}\left(\omega^{\prime}, Y^{\prime}\right) \neq 0$. Then we may assume that $\beta(\omega)$ in the assumption (1.19) is smooth and satisfies $|\beta(\omega)|=1$ in some neighborhood $\mathcal{O}\left(\subset S^{2}\right)$ of $\omega^{\prime}$. Indeed, there exists a neighborhood $\mathcal{O}$ of $\omega^{\prime}$ such that we have $F^{\text {red }}\left(\omega, Y^{\prime}\right) \neq 0$ for $\omega \in \mathcal{O}$. We define $\widetilde{\beta}(\omega)=\left|F^{\text {red }}\left(\omega, Y^{\prime}\right)\right|^{-1} F^{\text {red }}\left(\omega, Y^{\prime}\right)$. Then $\widetilde{\beta}(\omega)$ is a smooth function of $\omega \in \mathcal{O}$, and apparently satisfies $|\widetilde{\beta}(\omega)|=1$. Moreover, from (1.19) we see that $\widetilde{\beta}(\omega)$ is proportional to $\beta(\omega)$, and (1.21) as well as (1.19) (with modified $M(\omega, Y))$ remains valid if we replace $\beta(\omega)$ by $\widetilde{\beta}(\omega)$ for $\omega \in \mathcal{O}$.

Since $|\beta(\omega)|=1$ for $\omega \in \mathcal{O}$, it follows from (1.19), (1.20), and (1.17) that

$$
M(\omega, Y)=\left\langle\beta(\omega), F^{\mathrm{red}}(\omega, Y)\right\rangle=\sum_{m=1}^{N} \sum_{k=1}^{N} \sum_{l=1}^{N_{0}} \beta_{m}(\omega) g_{m l, k}(\omega) Y_{k} h_{l}(\omega, Y)
$$

for $(\omega, Y) \in \mathcal{O} \times \mathbb{R}^{N}$. Thus, again by (1.19), we obtain

$$
F_{j}^{\mathrm{red}}(\omega, Y)=\beta_{j}(\omega) \sum_{m=1}^{N} \sum_{k=1}^{N} \sum_{l=1}^{N_{0}} \beta_{m}(\omega) g_{m l, k}(\omega) Y_{k} h_{l}(\omega, Y)
$$

for $(\omega, Y) \in \mathcal{O} \times \mathbb{R}^{N}$ and $1 \leq j \leq N$. We fix a neighborhood $\mathcal{O}^{\prime}$ of $\omega^{\prime}$, which is strictly contained in $\mathcal{O}$, and choose a smooth cut-off function $\chi=\chi(\omega)$ satisfying $\chi \equiv 1$ on $\mathcal{O}^{\prime}$ and $\chi \equiv 0$ on $S^{2} \backslash \mathcal{O}$. From (1.20) and (8.18), we have

$$
F_{j}^{\mathrm{red}}(\omega, Y)=\sum_{l=1}^{N_{0}} \widetilde{g}_{j l}(\omega, Y) h_{l}(\omega, Y), \quad 1 \leq j \leq N,(\omega, Y) \in S^{2} \times \mathbb{R}^{N},
$$

where

$$
\widetilde{g}_{j l}(\omega, Y)=\sum_{k=1}^{N} \widetilde{g}_{j l, k}(\omega) Y_{k}, \quad 1 \leq j \leq N, 1 \leq l \leq N_{0}
$$

with

$$
\widetilde{g}_{j l, k}(\omega)=(1-\chi(\omega)) g_{j l, k}(\omega)+\chi(\omega) \beta_{j}(\omega) \sum_{m=1}^{N} \beta_{m}(\omega) g_{m l, k}(\omega)
$$

for $1 \leq j, k \leq N$ and $1 \leq l \leq N_{0}$. Thus (1.20) holds true if we replace $g_{j l, k}$ by $\tilde{g}_{j l, k}$. We consider the extended system (4.6), and apply Theorem 2.6 to it in the following way: Let $G$ be defined by (4.15)-(4.16) with $g_{j l, k}$ being replaced by $\widetilde{g}_{j l, k}$, and let $\mathbf{A}[\zeta]=\left(A_{j k}[\zeta]\right)_{1 \leq j, k \leq 5 N}$ be determined by (2.19)-(2.20) (with $N^{\prime}=5 N$ and $\left.N^{\prime \prime}=N_{0}\right)$ correspondingly to this replaced $G$, where $\zeta=\left(\zeta_{l}\right)_{1 \leq l \leq N_{0}}^{\mathrm{T}}$ is a smooth function of $(\sigma, \omega) \in \mathbb{R} \times S^{2}$. Then, for $1 \leq j \leq N$ we get

$$
A_{j k}[\zeta](\sigma, \omega)= \begin{cases}-\frac{1}{2} \sum_{l=1}^{N_{0}} \widetilde{g}_{j l, k}(\omega) \zeta_{l}(\sigma, \omega), & 1 \leq k \leq N \\ 0, & N+1 \leq k \leq 5 N .\end{cases}
$$


We define $\mathbf{B}(\omega, \eta)=\left(B_{j k}(\omega, \eta)\right)_{1 \leq j, k \leq N}$ by

$$
\mathbf{B}(\omega, \eta)=-\frac{1}{2} \beta(\omega) \eta^{\mathrm{T}} \sum_{m=1}^{N} \beta_{m}(\omega) \mathbf{G}_{m}(\omega), \quad(\omega, \eta) \in \mathcal{O} \times \mathbb{R}^{N_{0}},
$$

where $\mathbf{G}_{1}, \ldots, \mathbf{G}_{N}$ are $N_{0} \times N$ matrix-valued functions defined by

$$
\mathbf{G}_{j}(\omega)=\left(g_{j l, k}(\omega)\right)_{1 \leq l \leq N_{0}, 1 \leq k \leq N}, \quad 1 \leq j \leq N .
$$

Then (8.19) yields

$$
A_{j k}[\zeta](\sigma, \omega)=B_{j k}(\omega, \zeta(\sigma, \omega)), \quad 1 \leq j, k \leq N,(\sigma, \omega) \in \mathbb{R} \times \mathcal{O}^{\prime} .
$$

Now we apply Theorem 2.6 to the extended system (4.6) of $u^{*}$ with $5 N+N_{0}$ components, and we look only at the first $N$ components corresponding to the original $u$, and the last $N_{0}$ components corresponding to $w$ which is defined by (4.3). Then we see that there exist $U=\left(U_{j}(\sigma, \omega)\right)_{1 \leq j \leq N}^{\mathrm{T}}$ and $W=\left(W_{k}(\sigma, \omega)\right)_{1 \leq k \leq N_{0}}^{\mathrm{T}}$ such that

$$
\begin{aligned}
& \sum_{a=0}^{3}\left|r\left(\partial_{a} u\right)(t, r \omega)-\varepsilon \omega_{a} e^{(\varepsilon \log t) \mathbf{B}(\omega, W(r-t, \omega))}\left(\partial_{\sigma} U\right)(r-t, \omega)\right| \\
& \leq C \varepsilon\langle t+r\rangle^{3 \lambda+C \varepsilon-1}, \quad \omega \in \mathcal{O}^{\prime}, \\
& |r w(t, r \omega)-\varepsilon W(r-t, \omega)| \leq C \varepsilon\langle t+r\rangle^{2 \lambda-1}\langle t-r\rangle^{1-\rho}, \quad \omega \in S^{2}
\end{aligned}
$$

for $(t, r)$ satisfying $r \geq t / 2 \geq 1$, where $\lambda$ and $\rho$ are positive constants as in Theorem 2.5. Moreover, correspondingly to (2.24) we also have

$$
\sum_{j=1}^{N}\left|\partial_{\sigma} U_{j}(\sigma, \omega)-\partial_{\sigma} \mathcal{F}_{0}\left[f_{j}, g_{j}\right](\sigma, \omega)\right| \leq C \varepsilon(1+|\sigma|)^{3 \lambda+C \varepsilon-1} .
$$

Let $\mathbf{H}$ be a matrix-valued function given by

$$
\mathbf{H}(\omega)=\left(\sum_{a=0}^{3} h_{l, k a} \omega_{a}\right)_{1 \leq l \leq N_{0}, 1 \leq k \leq N},
$$

where $h_{l, k a}$ is from (1.18). Then the condition (1.21) leads to

$$
\mathbf{H}(\omega) \beta(\omega)=0, \quad \omega \in S^{2} .
$$

We claim that

$$
W(\sigma, \omega)=\mathbf{H}(\omega)\left(\partial_{\sigma} U\right)(\sigma, \omega)=\mathbf{H}(\omega) \Upsilon(\tau, \sigma, \omega)
$$

for $(\sigma, \omega) \in \mathbb{R} \times \mathcal{O}^{\prime}$ and $\tau \in \mathbb{R}$, where $\Upsilon(\tau, \sigma, \omega)=e^{\tau \mathbf{B}(\omega, W(\sigma, \omega))}\left(\partial_{\sigma} U\right)(\sigma, \omega)$. From (8.24) and (8.20), we get

$$
\mathbf{H}(\omega) \mathbf{B}(\omega, \eta)=0, \quad(\omega, \eta) \in \mathcal{O}^{\prime} \times \mathbb{R}^{N_{0}},
$$

and we obtain

$$
\mathbf{H}(\omega) e^{\tau \mathbf{B}(\omega, \eta)}=\mathbf{H}(\omega), \quad(\tau, \omega, \eta) \in \mathbb{R} \times \mathcal{O}^{\prime} \times \mathbb{R}^{N_{0}},
$$


which leads to

$$
\mathbf{H}(\omega) \Upsilon(\tau, \sigma, \omega)=\mathbf{H}(\omega)\left(\partial_{\sigma} U\right)(\sigma, \omega), \quad(\tau, \sigma, \omega) \in \mathbb{R} \times \mathbb{R} \times \mathcal{O}^{\prime} .
$$

From (8.26), (4.3), and (8.21), we get

$$
\begin{aligned}
\left|r w(t, r \omega)-\varepsilon \mathbf{H}(\omega)\left(\partial_{\sigma} U\right)(r-t, \omega)\right| & =|r w(t, r \omega)-\varepsilon \mathbf{H}(\omega) \Upsilon(\varepsilon \log t, r-t, \omega)| \\
& \leq C \varepsilon\langle t+r\rangle^{3 \lambda+C \varepsilon-1} .
\end{aligned}
$$

By (8.22) and (8.27), we get

$$
\left|W(\sigma, \omega)-\mathbf{H}(\omega)\left(\partial_{\sigma} U\right)(\sigma, \omega)\right| \leq \lim _{t \rightarrow \infty} C\langle 2 t+\sigma\rangle^{3 \lambda+C \varepsilon-1}=0, \quad(\sigma, \omega) \in \mathbb{R} \times \mathcal{O}^{\prime},
$$

which, together with (8.26), shows (8.25).

We put

$$
\mathbf{C}(\omega)=-\frac{1}{2} \sum_{m=1}^{N} \beta_{m}(\omega) \mathbf{G}_{m}(\omega)^{\mathrm{T}} \mathbf{H}(\omega), \quad \omega \in \mathcal{O}^{\prime}
$$

so that we have

$$
\mathbf{B}(\omega, \mathbf{H}(\omega) Y)=\beta(\omega)(\mathbf{C}(\omega) Y)^{\mathrm{T}}, \quad \omega \in \mathcal{O}^{\prime} .
$$

Observing that (1.18) can be written as $\left(h_{l}(\omega, Y)\right)_{1 \leq l \leq N_{0}}^{\mathrm{T}}=\mathbf{H}(\omega) Y$, we obtain from (8.18) and (8.20) that

$$
F^{\mathrm{red}}(\omega, Y)=-2 \mathbf{B}(\omega, \mathbf{H}(\omega) Y) Y=-2\langle\mathbf{C}(\omega) Y, Y\rangle \beta(\omega), \quad(\omega, Y) \in \mathcal{O}^{\prime} \times \mathbb{R}^{N} .
$$

Hence, recalling $F^{\text {red }}\left(\omega^{\prime}, Y^{\prime}\right) \neq 0$, and taking smaller $\mathcal{O}^{\prime}$ if necessary, we may assume that

$$
\left\langle\mathbf{C}(\omega) Y^{\prime}, Y^{\prime}\right\rangle \neq 0 \text { for any } \omega \in \mathcal{O}^{\prime}
$$

First we assume that there exists some $\left(\omega^{*}, Y^{*}\right) \in \mathcal{O}^{\prime} \times \mathbb{R}^{N}$ such that

$$
\left\langle\mathbf{C}\left(\omega^{*}\right) Y^{*}, \beta\left(\omega^{*}\right)\right\rangle \neq 0
$$

Then in combination with (8.28) for $\omega=\omega^{*}$, Lemma 8.4 implies that there exists $Y^{0} \in \mathbb{R}^{N}$ satisfying

$$
\left\langle\mathbf{C}\left(\omega^{*}\right) Y^{0}, Y^{0}\right\rangle \neq 0 \text { and }\left\langle\mathbf{C}\left(\omega^{*}\right) Y^{0}, \beta\left(\omega^{*}\right)\right\rangle>0 .
$$

We put

$$
\lambda_{0}=\left\langle\mathbf{C}\left(\omega^{*}\right) Y^{0}, \beta\left(\omega^{*}\right)\right\rangle, \mu_{0}=\left\langle\mathbf{C}\left(\omega^{*}\right) Y^{0}, Y^{0}\right\rangle .
$$

Note that (8.29) implies $\lambda_{0}>0$ and $\mu_{0} \neq 0$. Now fix some $\sigma_{0}>0$. By Lemma 7.3, we can choose some $C_{0}^{\infty}$-data such that we have

$$
\left(\left(\partial_{\sigma} \mathcal{F}_{0}\left[f_{j}, g_{j}\right]\right)\left(\sigma_{0}, \omega\right)\right)_{1 \leq j \leq N}^{\mathrm{T}}=Y^{0}, \quad \omega \in S^{2} .
$$


Let $U=U(\sigma, \omega ; \varepsilon)$ be the modified asymptotic profile corresponding to this choice of data. We set

$$
\begin{aligned}
\tilde{\boldsymbol{\Theta}}_{\varepsilon}(t, \sigma, \omega) & =(\varepsilon \log t) \mathbf{B}\left(\omega, \mathbf{H}(\omega)\left(\partial_{\sigma} U\right)(\sigma, \omega ; \varepsilon)\right), \\
\lambda_{\varepsilon}(\sigma, \omega) & =\left\langle\mathbf{C}(\omega)\left(\partial_{\sigma} U\right)(\sigma, \omega ; \varepsilon), \beta(\omega)\right\rangle, \\
\mu_{\varepsilon}(\sigma, \omega) & =\left\langle\mathbf{C}(\omega)\left(\partial_{\sigma} U\right)(\sigma, \omega ; \varepsilon),\left(\partial_{\sigma} U\right)(\sigma, \omega ; \varepsilon)\right\rangle .
\end{aligned}
$$

Since $\mathbf{C}$ and $\beta$ are smooth in $\mathcal{O}^{\prime}$, in view of (8.23) and (8.30) we get

$$
\lim _{(\sigma, \omega, \varepsilon) \rightarrow\left(\sigma_{0}, \omega^{*}, 0\right)} \lambda_{\varepsilon}(\sigma, \omega)=\lambda_{0}, \quad \lim _{(\sigma, \omega, \varepsilon) \rightarrow\left(\sigma_{0}, \omega^{*}, 0\right)} \mu_{\varepsilon}(\sigma, \omega)=\mu_{0}
$$

Hence there exist an open set $\mathcal{O}^{\prime \prime} \subset \mathcal{O}^{\prime}$ (with $\omega^{*} \in \mathcal{O}^{\prime \prime}$ ) and some open interval $\mathcal{I}\left(\ni \sigma_{0}\right)$ such that we have

$$
\begin{array}{ll}
\lambda_{0} / 2 \leq \lambda_{\varepsilon}(\sigma, \omega) \leq 3 \lambda_{0} / 2, & (\sigma, \omega) \in \mathcal{I} \times \mathcal{O}^{\prime \prime} \\
\left|\mu_{\varepsilon}(\sigma, \omega)\right| \geq\left|\mu_{0}\right| / 2>0, & (\sigma, \omega) \in \mathcal{I} \times \mathcal{O}^{\prime \prime}
\end{array}
$$

for small and positive $\varepsilon$. We may assume $\sigma>0$ for $\sigma \in \mathcal{I}$. Now (8.16) (with $p=\mathbf{C}(\omega)\left(\partial_{\sigma} U\right), q=\beta(\omega)$, and $\left.y=\partial_{\sigma} U\right)$ yields

$$
\begin{aligned}
\left|e^{\tilde{\boldsymbol{\Theta}}_{\varepsilon}(t, \sigma, \omega)}\left(\partial_{\sigma} U\right)(\sigma, \omega)\right| & =\left|\left(\partial_{\sigma} U\right)(\sigma, \omega)+\frac{\mu_{\varepsilon}(\sigma, \omega)}{\lambda_{\varepsilon}(\sigma, \omega)}\left(e^{\lambda_{\varepsilon}(\sigma, \omega) \tau}-1\right) \beta(\omega)\right| \\
& \geq \frac{\left|\mu_{0}\right|}{3 \lambda_{0}}\left(e^{\lambda_{0} \tau / 2}-1\right)-C \geq \frac{\left|\mu_{0}\right|}{6 \lambda_{0}} t^{\lambda_{0} \varepsilon / 2}
\end{aligned}
$$

for $(\sigma, \omega) \in \mathcal{I} \times \mathcal{O}^{\prime \prime}$ and $t \geq t_{\varepsilon}^{*}$, where we have put $\tau=\varepsilon \log t$, and $t_{\varepsilon}^{*}$ is some positive constant depending on $\varepsilon$. In view of (8.21), this shows that (AFP) does not hold. We define

$$
\widetilde{E}(t)^{2}:=\int_{t / 2}^{\infty}\left(\int_{S^{2}}\left|e^{\tilde{\Theta}_{\varepsilon}(t, r-t, \omega)}\left(\partial_{\sigma} U\right)(r-t, \omega)\right|^{2} d S_{\omega}\right) d r .
$$

By (8.32), we obtain

$$
\widetilde{E}(t) \geq\left(\frac{\mu_{0}^{2}}{36 \lambda_{0}^{2}} \int_{\mathcal{I}} \int_{\mathcal{O}^{\prime \prime}} t^{\lambda_{0} \varepsilon} d S_{\omega} d \sigma\right)^{1 / 2} \geq C t^{\lambda_{0} \varepsilon / 2}
$$

for $t \geq t_{\varepsilon}^{*}$. From Corollary 7.1 and (8.33), we find that $\|u(t)\|_{E} \geq C \varepsilon(1+t)^{C \varepsilon}$ for $t \geq t_{\varepsilon}^{* *}$ with some $t_{\varepsilon}^{* *}>0$, and (AFE) never holds.

Next we assume that

$$
\langle\mathbf{C}(\omega) Y, \beta(\omega)\rangle=0
$$

holds for all $(\omega, Y) \in \mathcal{O}^{\prime} \times \mathbb{R}^{N}$. Fix some $\omega^{*} \in \mathcal{O}^{\prime}$, and some $\sigma_{0}>0$. Because of (8.28), if we put $Y^{0}=Y^{\prime}$, we have $\left\langle\mathbf{C}\left(\omega^{*}\right) Y^{0}, Y^{0}\right\rangle \neq 0$. Now we choose some data such that we have (8.30). As above, we can also choose some $\mathcal{O}^{\prime \prime}\left(\subset \mathcal{O}^{\prime}\right)$ and some 
interval $\mathcal{I}\left(\ni \sigma_{0}\right)$ such that (8.31) holds for small $\varepsilon$. Then, from (8.16) we obtain

$$
\begin{aligned}
\left|e^{\tilde{\boldsymbol{\Theta}}_{\varepsilon}(t, \sigma, \omega)}\left(\partial_{\sigma} U\right)(\sigma, \omega ; \varepsilon)\right| & =\left|\left(\partial_{\sigma} U\right)(\sigma, \omega ; \varepsilon)+(\varepsilon \log t) \mu_{\varepsilon}(\sigma, \omega) \beta(\omega)\right| \\
& \geq \frac{\left|\mu_{0}\right|}{2} \varepsilon \log t-C \geq \frac{\left|\mu_{0}\right|}{4} \varepsilon \log t
\end{aligned}
$$

for $(\sigma, \omega) \in \mathcal{I} \times \mathcal{O}^{\prime \prime}$ and $t \geq t_{\varepsilon}^{*}$ with some $t_{\varepsilon}^{*}>0$. From (8.34) we conclude that (AFP) fails to hold. Finally (8.34) leads to $\widetilde{E}(t) \geq C \varepsilon \log t$ for $t \geq t_{\varepsilon}^{*}$, and we find that (AFE) does not hold because we get $\|u(t)\|_{E} \geq C \varepsilon^{2} \log t$ for $t \geq t_{\varepsilon}^{* *}$ with some $t_{\varepsilon}^{* *}>0$. This completes the proof.

\section{ACKNowledgments}

The author would like to express his gratitude to Professor Yoshio Tsutsumi, because the present work is motivated by his question during the author's talk. The author is grateful to Professor Serge Alinhac for his helpful suggestions, and to Professor Hideaki Sunagawa for fruitful conversations.

This research is partially supported by Grant-in-Aid for Scientific Research (C) (No. 20540211), Japan Society for the Promotion of Science.

\section{REFERENCES}

[1] S. Alinhac, An example of blowup at infinity for a quasilinear wave equation, Astérisque $\mathbf{2 8 4}$ (2003), 1-91.

[2] S. Alinhac, Remarks on energy inequalities for wave and Maxwell equations on a curved background, Math. Ann. 329 (2004), 707-722.

[3] S. Alinhac, Semilinear hyperbolic systems with blowup at infinity, Indiana Univ. Math. J. 55 (2006), 1209-1232.

[4] S. Asakura, Existence of a global solution to a semi-linear wave equation with slowly decreasing initial data in three space dimensions, Comm. Partial Differential Equations 11 (1986), 1459-1487.

[5] D. Christodoulou, Global solutions of nonlinear hyperbolic equations for small initial data, Comm. Pure Appl. Math. 39 (1986), 267-282.

[6] L. Hörmander, The lifespan of classical solutions of non-linear hyperbolic equations, in Pseudo-differential Operators, Lecture Notes in Math. Vol. 1256 (Springer-Verlag, Berlin, 1987), pp. 214-280.

[7] L. Hörmander, Lectures on Nonlinear Hyperbolic Differential Equations (Springer-Verlag, Berlin, 1997).

[8] F. John, Blow-up of solutions of nonlinear wave equations in three space dimensions, Manuscripta Math. 28 (1979), 235-268.

[9] F. John, Blow-up of solutions for quasi-linear wave equations in three space dimensions, Comm. Pure Appl. Math. 34 (1981), 29-51.

[10] F. John, Lower bounds for the life span of solutions of nonlinear wave equations in three space dimensions, Comm. Pure Appl. Math. 36 (1983), 1-35.

[11] F. John, Existence for large times of strict solutions of nonlinear wave equations in three space dimensions for small initial data, Comm. Pure Appl. Math. 40 (1987), 79-109.

[12] S. Katayama and H. Kubo, Asymptotic behavior of solutions to semilinear systems of wave equations, Indiana Univ. Math. J. 57 (2008), 377-400.

[13] S. Katayama and H. Kubo, The rate of convergence to the asymptotics for the wave equation in an exterior domain, Funkcial. Ekvac. 53 (2010), 331-358. 
[14] S. Katayama and K. Yokoyama, Global small amplitude solutions to systems of nonlinear wave equations with multiple speeds, Osaka J. Math. 43 (2006), 283-326.

[15] S. Klainerman, Uniform decay estimates and the Lorentz invariance of the classical wave equation, Comm. Pure Appl. Math. 38 (1985), 321-332.

[16] S. Klainerman, The null condition and global existence to nonlinear wave equations, in Nonlinear Systems of Partial Differential Equations in Applied Mathematics, Part 1, Lectures in Appl. Math. 23 (AMS, Providence, 1986), pp. 293-326.

[17] S. Klainerman, Remarks on the global Sobolev inequalities in the Minkowski space $\mathbf{R}^{n+1}$, Comm. Pure Appl. Math. 40 (1987), 111-117.

[18] K. Kubota and K. Yokoyama, Global existence of classical solutions to systems of nonlinear wave equations with different speeds of propagation, Japan. J. Math. (N.S.) 27 (2001), 113-202.

[19] P. D. Lax and R. S. Phillips, Scattering Theory, Revised Edition (Academic Press, New York, 1989).

[20] H. Lindblad, On the lifespan of solutions of nonlinear wave equations with small initial data, Comm. Pure Appl. Math. 43 (1990), 445-472.

[21] H. Lindblad and I. Rodnianski, The weak null condition for Einstein's equations, C. R. Math. Acad. Sci. Paris 336 (2003), 901-906.

[22] H. Lindblad and I. Rodnianski, Global existence for the Einstein vacuum equations in wave coordinates, Commun. Math. Phys. 256 (2005), 43-110.

[23] H. Sunagawa, A note on the large time asymptotics for a system of Klein-Gordon equations, Hokkaido Math. J. 33 (2004), 457-472.

[24] H. Sunagawa, Large time asymptotics of solutions to nonlinear Klein-Gordon systems, Osaka J. Math. 42 (2005), 65-83.

DePARTMEnt of Mathematics, WaKayama University

930 SAKAEDANI, WAKAYAMA 640-8510, JAPAN

e-mail: katayama@center.wakayama-u.ac.jp 